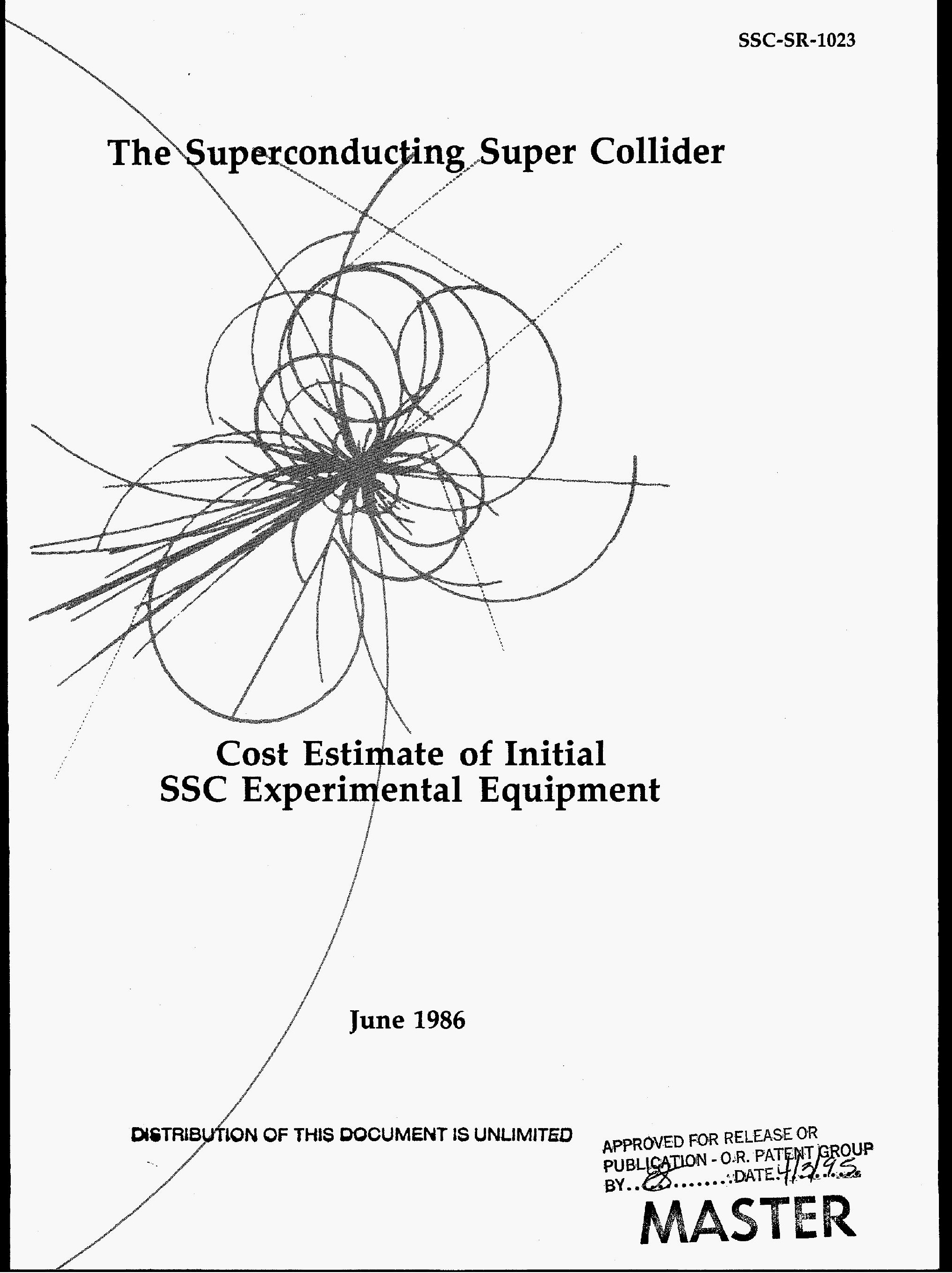




\title{
COST ESTIMATE OF INITIAL SSC EXPERIMENTAL EQUIPMENT
}

\author{
SSC Central Design Group* \\ c/o Lawrence Berkeley Laboratory \\ Berkeley, California 94720
}

June 1986

\section{DISCLAIMER}

\begin{abstract}
This report was prepared as an account of work sponsored by an agency of the United States Government. Neither the United States Government nor any agency thereof, nor any of their employees, makes any warranty, express or implied, or assumes any legal liability or responsibility for the accuracy, completeness, or usefulness of any information, apparatus, product, or process disclosed, or represents that its use would not infringe privately owned rights. Reference herein to any specific commercial product, process, or service by trade name, trademark, manufacturer, or otherwise does not necessarily constitute or imply its endorsement, recommendation, or favoring by the United States Government or any agency thereof. The views and opinions of authors expressed herein do not necessarily state or reflect those of the United States Government or any agency thereof.
\end{abstract}




\section{DISCLAIMER}

Portions of this document may be illegible in electronic image products. Images are produced from the best available original document. 
Cost Estimate of the Initial SSC Experimental Equipment

Attachment A: Report of the Detector Cost Model Advisory Panel - . 9

I. Introduction . . . . . . . . . . . . . . . . 11

II. Qualifications and Assumptions . • . • . . • . . . 12

III. Overview of Detector Scenarios . • . • . . . . . . 13

IV. Detailed Description of Detectors . • . . . . . . . 18

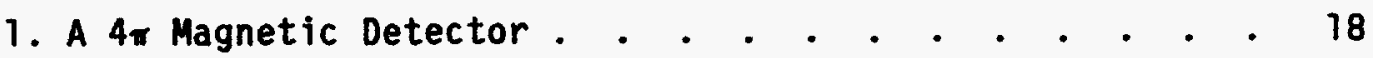

2. Muon Spectrometers Matched to the Full

Kinematic Range of the SSC . . . . . . . . . 30

3. Upgrade $4 \pi$ Detector with a Forward Spectrometer . . . 43

Appendix I: Initial Detector Complement Advisory Panel • • . 83

Appendix II: A Modular Multidrift Vertex Detector . . • . 85

Attachment B: Report of the Detector Cost Evaluation Panel - . . 89

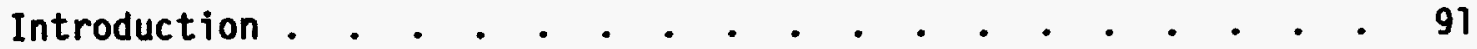

Conclusions and Recommendations . . . . . . . . . . . 92

Cost Assumptions . . . . . . . . . . . . . . . . 94

Cost Estimates . . . . . . . . . . . . . . . . . 97

General Comments . . . . . . . . . . . . . . . . 97

Comparison with Snowmass 84 . . . . . . . . . . . . . 99

Appendix A: SSC Detector Cost Evaluation Panel . . . . . . 101

Appendix B: Detector Spreadsheets . . . . . . . . . . 103

Attachment C: Report of the Off-line Computing Advisory Panel • 115

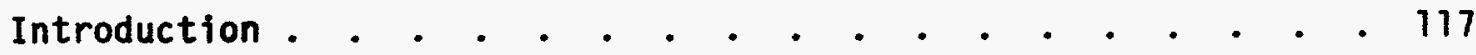

Requirements . . • . • • . • • • • • • • • • • • 119

Conventional Computing . . . . . . . . . . . . . . 123

Processor Farms . . . . . . . . . . . . . . . . 130

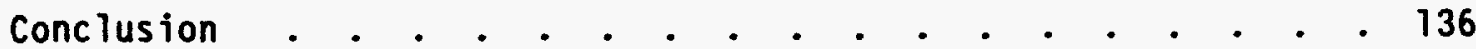

Appendix A: Off-line Computing Advisory Panel • . . . . . 137

Appendix B: Requirement for SSC Central Computing Staffing . . 139 


\section{Introduction}

The cost of the initial detector complement at recently constructed colliding beam facilities (or at those under construction) has been a significant fraction of the cost of the accelerator complex. Because of the complexity of large modern-day detectors, the time-scale for their design and construction is comparable to the time-scale needed for accelerator design and construction. For these reasons it is appropriate to estimate the cost of the anticipated detector complement in parallel with the cost estimates of the collider itself.

The fundamental difficulty with this procedure is that, whereas a firm conceptual design of the collider does exist, comparable information is unavailable for the detectors. Traditionally, these have been built by the high energy physics user community according to their perception of the key scientific problems that need to be addressed. The role of the accelerator laboratory in that process has involved technical and managerial coordination and the allocation of running time and local facilities among the proposed experiments. It seems proper that the basic spirit of experimentation reflecting the scientific judgment of the community should be preserved at the SSC. Furthermore, the formal process of initiation of detector proposals can only start once the SSC has been approved as a construction project and a formal laboratory administration put in place. Thus an ad hoc mechanism had to be created to estimate the range of potential detector needs, potential detector costs, and associated computing equipment. 
In spite of the limitations mentioned above, reasonable bounds on costs of the SSC detectors can be estimated because those detectors represent a natural evolution of the instrumentation in use today. The basic techniques of fabricating large detectors for use at particle colliders are by now well established and understood. The process of data reduction and extraction of physics from raw data is also well defined by past experience. Even though the SSC will represent a significant jump in both energy and luminosity, various studies and workshops have led to the conclusion that detectors for the SSC can be built with modest extensions of the existing technology. Further development in instrumentation may lead to better technology and lower costs, and thus may reduce the costs from those quoted in the accompanying reports.

This report describes the process that has been generated to provide the cost estimate for the experimental equipment at the SSC, and the results of this process. Equally important, however, is the discussion of the basic limitations of this process and, hence, of the results. The procedural details, as well as the backup material for the numbers obtained, can be found in the reports of the three relevant advisory panels that are submitted as attachments.

\section{Procedure}

Three advisory panels of experts from the world community of particle physicists (membership shown in the attached reports) were appointed to consider SSC detector requirements and to generate the required cost estimates: 
1) The Detector Cost Model Advisory Panel (DCMAP) was convened to put forth a reasonable model of what an initial detector complement might look like. This pane1, chaired by Professor George Trilling of the University of California, Berkeley, provided a report that was then used as input for the work of the other panels.

2) The Detector Cost Evaluation Panel (DCEP), chaired by Professor Roy Schwitters of Harvard University, was asked to estimate the costs for that complement of detectors. In its work the panel used the cost data base obtained from large detectors presently under construction (CDF, DO, L3, SLD) and made some assumptions about the future cost trends for electronics and data acquisition equipment.

3) The off-line Computer Advisory Panel (OCAP), chaired by Dr. Stewart Loken of Lawrence Berkeley Laboratory, was charged with estimating the cost of the off-line computer facility at SSC. The data output of the detector complement generated by DCMAP was used to provide an estimate of the computing load at the SSC.

\section{The Model Adopted}

The initial detector complement model suggested by the first panel consisted of the following detectors:

1) a large 4r al1-purpose magnetic detector;

2) a spectrometer for high energy muons;

3) an upgraded $4 \pi$ detector, existing or in construction at the present time, combined with a precision forward spectrometer; 
4) apparatus to measure total and elastic scattering cross sections. DCMAP also suggested that some funds be held in reserve for other rather modest experiments incorporating new ideas that might be put forth later on.

The significant feature of this model is the distinct character of different detectors. This reflects the nature of physics at the hadron collider and contrasts with $\mathrm{e}^{+} \mathrm{e}^{-}$colliders, where all-purpose $4 \pi$ detectors are the norm and specialized detectors the exception. It illustrates the much greater potential variety of instrumentation at the SSC, and suggests that tradeoffs between relative costs and potential physics impact will be an important input in the future discussions about the actual SSC program.

\section{Cost Estimates}

The calculated range of costs for the DCMAP detector complement extends from $\$ 558 \mathrm{M}$ to $\$ 865 \mathrm{M}$. This range allows for the variation in the detector parameters suggested by DCMAP as well as for uncertainties in cost projections for different components.

The parallel exercise by OCAP results in a figure of $\$ 71 \mathrm{M}$ for total off-line computer facilities (including $\$ 5 \mathrm{M}$ for software). A significant increase in computing power per dollar is obtained by a relatively heavy reliance on microprocessor farms.

While the computing costs form a relatively small fraction of the total equipment cost, and are probably not subject to great uncertainty, the detector costs present quite a different picture. Thus several points need to be emphasized about the inherent limitations of the process adopted. 
The detector complement adopted represents an ambitious possible model for the initial set of detectors. Some parts of it may be more representative of what might be expected after a few years of gradual evolution and upgrading of the detectors. There has been no careful optimization of physics output per detector dollar in either the choice or the design of the detectors. The estimated costs are based mainly on existing cost data, with some allowance for future decrease in the cost of electronics and data acquisition systems due to further technological developments. There may be further savings from new inventions, standardization, pooling of resources and quantity discounts which should be more substantial than in the past. Clearly, these kinds of questions can only be addressed within the framework of an actual design effort.

The costing process used here follows the conventional bookkeeping procedures in use today for costing the detectors. Several recommendations about possible changes are enumerated in the DCEP report.

\section{Estimate of foreign contributions}

The last decade has seen extensive foreign participation in the fabrication of major detectors at U.S. accelerators, and equally large American participation in fabrication of detectors for use abroad. Without any additional formal agreements regarding foreign collaboration on the SSC, we would expect this pattern to continue for SSC detectors. An increase in the level of collaboration might be expected because the SSC would represent a unique facility in the world and would offer the only way to approach some of 
the most interesting problems in high energy physics. In other words, the SSC would be a true world facility, serving the international community of scientists.

The Collider Detector at Fermilab (CDF) can be used as a credible model for the level of foreign participation since it is built for the exploitation of a new facility at a hitherto unachieved energy. The funds contributed by Japan and Italy to the fabrication of CDF amount to $\$ 18 \mathrm{M}$, compared to the total U.S. contribution (DOE and NSF) of $\$ 46 M$.

It is possible that some of the formal international collaboration discussions that are taking place now will result in a more significant foreign participation in the SSC. Such additional foreign contributions could go into larger shares of detector costs and/or participation in other phases of the SSC (RED, construction, or operation).

\section{Comparison with previous cost estimates}

The work described in the attached reports probably represents the most careful cost estimate of experimental equipment for the SSC that has been performed to date. A rough estimate of costs of three different $4 \pi$ detectors performed as part of the 1984 DPF Snowmass workshop yielded a cost range per detector (before contingency) of $\$ 164 M$ to $\$ 210 M$ in FY84 $\$$, to be compared with DCEP cost estimate of a magnetic $4 \pi$ detector of $\$ 290-334 M$ in FY86 \$. The Snowmass cost estimates did not include an allowance for EDIA, taken to be 15-20\% of the total in DCEP work, and were based on a much lower number of electronics channels. 
The 1985 HEPAP summer study estimated an annual expenditure for SSC equipment (full detector complement and computers) of $\$ 130 \mathrm{M}$ during the peak of SSC construction. As far as detectors are concerned, a 4 to 5 year fabrication period (and, hence, spending period at that level) might be anticipated, yielding a total equipment cost of \$520-650M.

Regarding the computer costs, a workshop at Fermilab late in 1985 on triggers, data acquisition and computing produced a very rough preliminary cost estimate of $\$ 45 M$ for off-line computing equipment.

We would interpret all of these cost numbers as reasonably self-consistent, with the spread indicating the level of uncertainty associated with these estimates at the present time. A large fraction of this uncertainty comes about because no specific decisions as to the actual detector design have been made, and thus at present several different possibilities may be entertained.

\section{Conclusions}

Any effort to estimate the cost of experimental equipment for the SSC at this time will suffer from many uncertainties. Thus the figures quoted have less significance than those in the Conceptual Design Report for the accelerator complex itself. On the other hand, it is our belief, based on past experience with other detectors, that a first rate experimental program at the SSC can be generated within the cost estimates given.

The DCEP estimate of the total cost (without contingency) of the initial complement of detectors is $\$ 558 \mathrm{M}$ to $\$ 865 \mathrm{M}$. To this figure one should add an OCAP estimate of off-line computing equipment costs of $\$ 71 \mathrm{M}$. According to 
accepted practice in estimating detector equipment costs, software development and detector R\&D costs have not been included. At this initial stage of planning we have not included any formal contingency because of our belief that it can be absorbed by adjustments in the total scope of the experimental program. Finally, to obtain the cost to the U.S. community one should subtract the anticipated foreign contributions. The range of costs quoted represents potential variation in the scope of the detectors and uncertainties in cost projections.

In summary, we believe that the described detector complement will allow a sound initial experimental program at the SSC to be mounted within the cost range quoted above. The program and the detectors outlined by the DCMAP are quite ambitious, but we believe that the detectors can be built using modest extensions of the existing technology. Some phasing-in may be required, and might be advisable on physics grounds. Thus the detectors at the initial SSC turn-on time may not need to be as extensive as described here. Foreign collaborators can be expected to provide a significant fraction of the total detector cost. 
February 1986 


\section{INTRODUCTION}

In October 1985 SSC-COG Director M. Tigner appointed the Detector-CostModel Advisory Panel and gave it the following charge:

The Initial-Detector-Complement Advisory Panel shall advise the Director of the Central Design Group about possible detectors that might be built, modified or moved for the initial exploitation of the SSC. The purpose of this recommendation is to provide the CDG with a possible scenario that could then be used to make cost estimates of the initial detector complement.

Some of the possibilities that the Panel should consider are: totally new detectors, completely finished; totally new detectors with significant upgrade possibilities; modifications of existing detectors; existing detectors without any significant modifications. Both $4 \pi$ and limited solid angle detectors should be considered. The Panel should attempt to strike an appropriate balance between the need to exploit the SSC and the necessity to keep costs within reasonable bounds.

The Panel should assume that four interaction regions will be developed initially, although comment on this assumption is appropriate. Possibility of special optics in some of them should not be excluded.

The Panel should finalize its recommendation by December 10, 1985.

The membership of the Panel is listed in Appendix 1. The Panel held two meetings: the first at LBL on November 18-19 and the second at Fermilab on December 10-11, 1985. The completion of its report, however, required more time than provided in the charge. The Panel had the benefit of technical advice from $A$. Garren of the CDG Group on issues related to interaction region (IR) design and machine performance, and from $D$. Groom on many issues related to SSC design parameters. 


\section{QUALIFICATIONS AND ASSUMPTIONS}

The choice of a detector complement, evolved over a relatively short time in response to our charge, necessarily has considerable arbitrariness. Reflecting our tastes and judgments, it probably bears little detailed resemblance to whatever program is eventually initiated when the SSC turns on. Its only function is to provide the basis for a cost estimate relevant to a group of detectors credibly matched to physics opportunities at SSC turn-on. We emphasize in the strongest possible terms that our activity bears no connection of any kind to the process through which an actual SSC program will eventually be formulated. We are simply proposing examples to help estimate costs.

We have borne in mind that a broad range of physics issues should be addressed in the SSC program. While the specific choices made here will undoubtedly not be the real ones, the general point is that the initial list of experiments will likely span a large range of physics, size, cost and impact upon the machine parameters.

We have given serious thought to the possibility of upgrades of existing detectors. Here two qualifications must be mentioned:

(1) Upgrade examples given in this report are products of the panel's efforts and have not been discussed nor approved by the collaborations involved in those detectors.

(2) We have not considered LEP or SLC detectors as upgrade candidates, on the grounds that the electron-positron programs for which they are intended will likely continue well beyond the time at which their installation at SSC would have to be initiated. In any case we believe that the hadron-collider detectors considered, CDF, DD and UAI, span a sufficient range of design choices to provide an adequate set of representative upgrade scenarios.

In developing candidate detector designs and parameter lists, we have made much use of the Snowmass-1984 and Lausanne Workshop Proceedings. We have assumed only limited extrapolations of present-day technology.

We believe that the IR configurations for our examples are or can be made compatible with the present SSC lattice plans, but detailed machine designs and calculations will need to be made. Since we foresee that the actual SSC experiments will be at least as demanding on IR parameters as the list given 
below, we urge continuing and close contact between machine physicists and experimenters on this subject.

Finally, as suggested in the charge, we have kept the issue of overall cost in mind to propose what we believe to be a detector complement matched to the physics opportunities in a reasonably cost effective way.

\section{OVERVIEW OF DETECTOR SCENARIOS}

Physics made accessible by an SSC of $40 \mathrm{TeV}$ energy and $10^{33} \mathrm{~cm}^{-2} \mathrm{~s}^{-1}$ luminosity has been discussed in many places, and we do not repeat these considerations here. We do want to note that, unlike $e^{t} e^{-}$colliders, which explore almost exclusively the energy scale corresponding to the machine energy, an SSC can profitably explore a vast range of different energy scales extending from several TeV down to a few GeV. For the highest energy scales detectors which emphasize the central region of rapidity and which are designed to handle the highest luminosity are appropriate. For lower energy scales, which can be studied with lower luminosities, detectors which emphasize the small-angle regions may be more economical and less massive.

Our choice of initial detector scenario has been motivated by the following considerations:

a. At turn-on there should be detectors capable of taking advantage of the full design luminosity and the highest mass scale.

b. There should be a variety of detector capabilities and designs.

c. The detector complement should span a large range of physics interests for SSC.

d. Not all IR space should be tied up with large and costly detectors, initiated many years before turn-on. There should be opportunities for smaller experiments, proposed and constructed over fairly short time scales by collaborations of modest size.

our explicit model for realizing these conditions consists of the following components:

1. A new magnetic $4 \pi$ detector designed to handle the full design luminosity for the study of physics at the highest mass scales made accessible by the SSC.

As discussed in the next section, one model for such a detector is the SCD 
Detector described in the Snowmass-1984 Proceedings. Some aspects such as vertex detection may have difficulties at the highest luminosities. However at the very least they will find application both in the earlier stages when the full design luminosity is not available, and in any specialized runs below full luminosity.

We believe that such a detector should be available at turn-on because it appears to be the instrument best suited for the general study of the TeV scale, the area which provides the major scientific justification for the SSC. It will undoubtedly take significant beam time to debug this detector, and its actual availability for physics will in any case be some time after machine turn-on. Furthermore, there may be substantial cost savings associated with building-in (as in the case of $L 3$ at LEP) such a large detector, rather than requiring that it be movable in and out of the beam. If "building-in" is desirable, the large installation time ( 2 years) makes construction before turn-on almost essential.

We have chosen a magnetic detector because: (1) this allows determination of the signs of high energy electrons (muons are emphasized in the next detector), and (2) this choice, coupled with the vertex detector, provides capabilities for flavor tagging, strange particle decay identification etc. which provide more complete information about events of interest.

2. A spectrometer for high energy muons designed for the highest luminosity operation.

We have considered two different designs, both intended to handle the full $10^{33} \mathrm{~cm}^{-2} \mathrm{~s}^{-1}$ design luminosity and to measure $1 \mathrm{TeV}$ muons with a precision of $5-10 \%$.

The more precise one is similar in spirit to the L3 muon spectrometer at LEP. The other is a large magnetized iron spectrometer. We have considered these somewhat specialized devices as credible candidates for initial operation for the following reasons: (1) accurate measurements of leptons may have high discovery potential, (2) the detectors do exploit the full machine luminosity and energy, (3) either one is quite different from the general $4 \pi$ detector, and (4) cost considerations may require, as in L3, that such a detector be built-in over a period of the order of two years prior to closure of the experimental halls. 
3. Upgraded $4 \pi$ detector with a forward spectrometer.

We believe that the upgrading of an existing detector could provide an experimental facility, reasonably matched to luminosities up to $10^{32} \mathrm{~cm}^{-2} \mathrm{~s}^{-1}$, which could rather rapidly get on the air to explore SSC physics soon after turn-on. There would already exist operating experience and software, and even the new components could perhaps be installed and checked out on the Tevatron or the SpD̄S prior to SSC installation, in the same spirit as the checkout of the upgraded MARK II at PEP. Vertex detectors, which appear marginal at $10^{33} \mathrm{~cm}^{-2} \mathrm{~s}^{-1}$, could probably be optimally used in the lower luminosity environment of the upgraded detector. We have considered upgrades of CDF, DO and UAT, and details are given in the next section. Each of these detectors bring particular strengths (tracking in a magnetic field for CDF, strong calorimetry in 00 , and dipole field configuration with efficient detection and measurement near the forward direction in UAT).

The study of physics in the forward region (rapidities $\geqslant 3$ ) requires an interaction region with considerably longer free space than the $\pm 20 \mathrm{~m}$ allocated to a high luminosity IR. High rates in this kinematic region compensate for the loss of luminosity due to the necessarily larger value of $B^{*}$. A forward spectrometer would permit the study of diffractive phenomena (including $W$ and $Z$ production at small angles). Furthermore the forward rapidity region can be studied with smaller detectors than is the case for the central region. We thus suggest a forward spectrometer covering the angular region from $60 \mathrm{mr}$ to about $1 \mathrm{mr}$, installed on one side of the IR with free space extending to $100 \mathrm{~m}$ from the luminous region. If $\beta^{\star}$ values down to 5 meters are possible, the available maximum luminosity would be about $10^{32} \mathrm{~cm}^{-2} \mathrm{~s}^{-1}$. This matches the luminosity needed for the upgraded $4 \pi$ detector, and a marriage of the two in one IR seems both economical and effective from the physics standpoint.

\section{4. otot, $\sigma_{\mathrm{el}}$, etc.}

The determination of $\sigma_{\text {tot }} \sigma_{e l}$, and the ratio of real to imaginary forward amplitude will almost surely have a strong physics interest early in the SSC operation. Required luminosities are small and the needed experimental equipment is also modest. The non-trivial requirements are (1): an IR with $\beta^{*} \sim 4 \mathrm{~km}$, with the possibility of varying $\beta^{*}$ downward by about a 
factor of 10, and (2) suitable locations for "Roman Pot" detectors, also at $\beta$ $\sim$ a few $\mathrm{km}$ on both sides of the IR. Furthermore protons scattered at the IR within the angular region of interest must be able to reach the detectors without being lost in a limiting aperture. It should be noted that this experiment will want to run at several beam energies between 1 and $20 \mathrm{TeV}$, with $\beta^{*}$ approximately proportional to energy. In the same IR one can also put in other fairly simple equipment to study multiplicities, correlations and other properties of minimum bias events. The experimental equipment costs are relatively small on the scale of the other detectors, but the machine and IR properties require study. This is an appropriate topic for a collaborative effort between interested physicists and machine experts.

\section{Other Experiments.}

We strongly believe that the SSC program should be flexible enough to insta 11, even at a rather late date, modest experiments which are different from the rather conventional devices that have been so far discussed. Of the six experimental IR's, only three have been required for the three major detectors plus the high $\beta^{\star}$ IR for $\sigma_{\text {tot }}$ etc. This leaves room for other experimentation. We recommend that, for purpose of cost estimation, an amount of the order of $10 \%$ of the support needed for the three large detectors be added to cover the costs of presently unpredictable, more modest experiments which smaller experimental groups will perhaps wish to install. This $10 \%$ figure should also amply cover the costs of the detectors needed for the $\sigma_{\text {tot }}$ etc. experiments.

We conclude this overview with some comments about IR properties assumed in our scenario. Fig. 1 shows a diagram of the proposed clustered IR's as presently envisaged, with an indication of where the suggested detectors would go. The two IR's on the left of the figure would be capable of maximum luminosity operation, but should be tunable downward through increase of local $\beta^{*}$ up to about a factor of 10 . One of the IR's on the right would have the $\pm 100 \mathrm{~m}$ free space and operation up to $10^{32} \mathrm{~cm}^{-2} \mathrm{~s}^{-1}$. We are not in a position to specify where the long $\beta^{*}$ IR should best go. Clearly further definition of the IR requirements and capabilities will require close cooperation between particle experimenters and machine experts. 


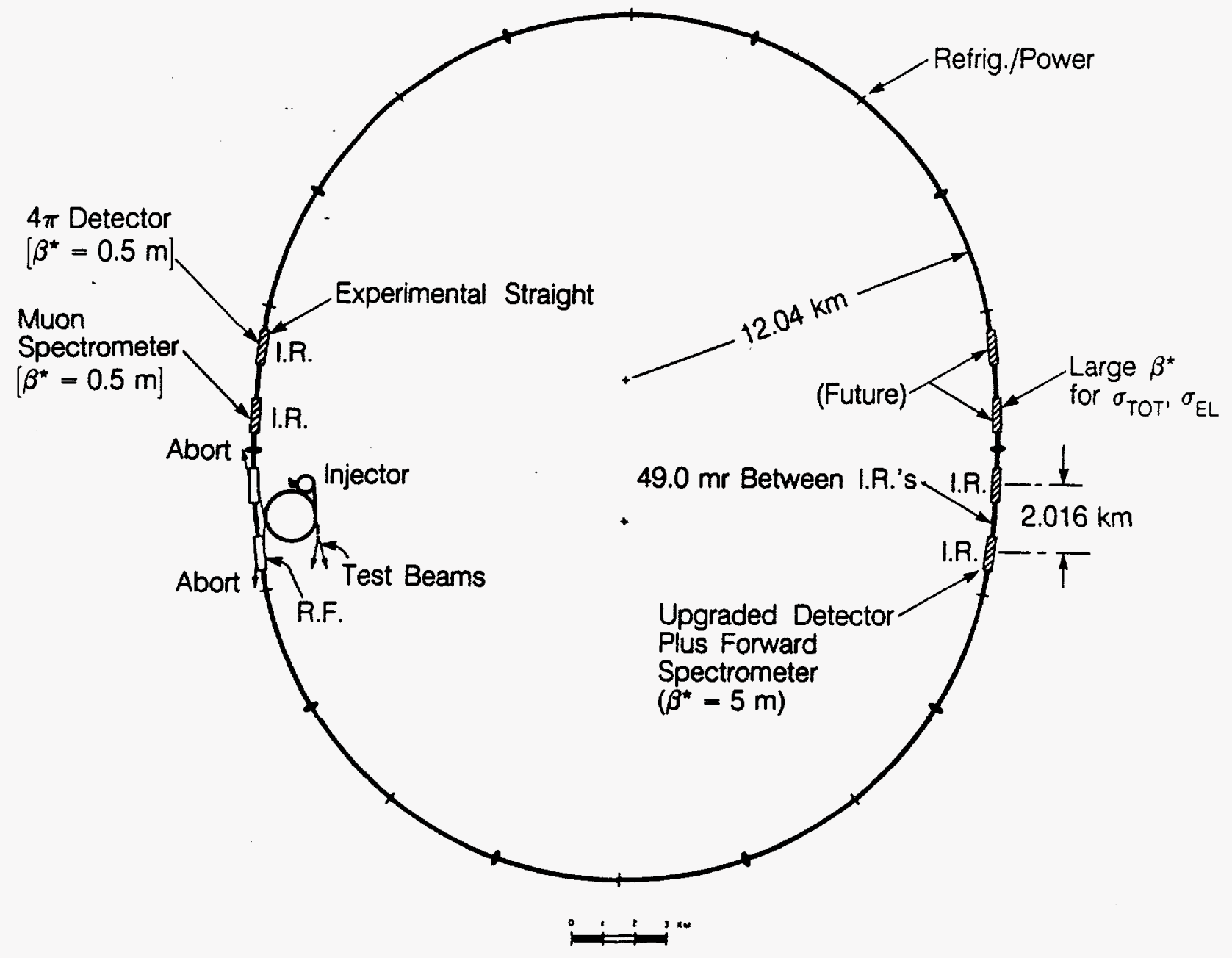

Fig. 1. Detector arrangement in I.R.'s. 


\section{DETAILED DESCRIPTION OF DETECTORS}

\section{A $4 \pi$ Magnetic Detector}

The design goals of a $4 \pi$ detector to explore the high mass range accessible at the SSC, include:

- hermetic calorimetric coverage over the rapidity range of at least

$|y|<5.5$, to permit accurate measurement of missing transverse energy,

- measurement capability of jet energies up to the multi-TeV range with good energy resolution,

- optimized efficiency for multi-jet detection and mass reconstruction of particles decaying into jets or jets and leptons,

- detection of muons over the rapidity range $|y|<5.0$ with the ability to determine at least crudely muon momenta at the trigger level over much of the same rapidity interval,

- optimal detection and identification of electrons via calorimetry in conjunction with tracking devices,

- momentum measurement capability for charged tracks in the central rapidity interval $|y|<2$, i.e., a magnetic detector,

- precision secondary vertex detection in at least the central rapidity interval for flavor tagging and the detection of relatively long-lived new states,

- some sensitivity to exotic new particles beyond a missing energy signature, and

- capability of operation at the SSC design luminosity $\left(10^{33} \mathrm{~cm}^{-2} \mathrm{sec}^{-1}\right)$ and at lower luminosities if desirable.

Implementation of these design goals in hardware and software will require some compromises. For cost estimating purposes we present two alternative designs of a $4 \pi$ solenoidal magnetic detector. One (Model A), essentially identical to that described in Snowmass -84 (p. 626-629, the SCD detector), is shown in Fig. 2. The second (Model B) is based somewhat more on the CDF detector and is shown in Fig. 3.

In the sections below we briefly describe the components of each of these model detectors and the assumptions made to determine the overall parameters. The relevant parameters are summarized in Tables 1 and 2 . There are obviously many uncertainties in the projections of the capabilities of the detector components. In particular, the feasibility of tracking devices at the SSC design 
A CONVENTIONAL MAGNETIC DETECTOR

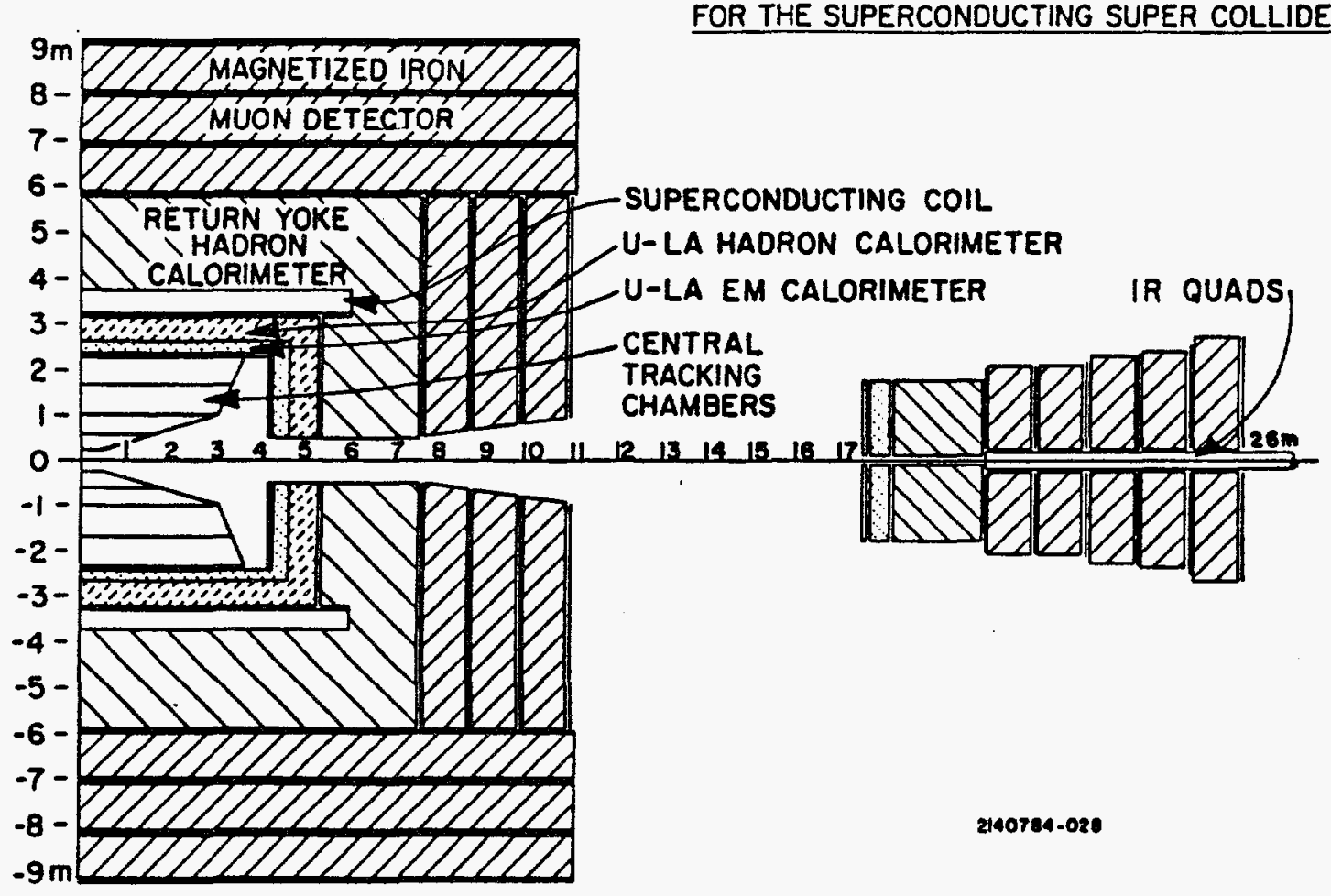

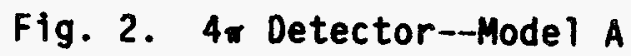




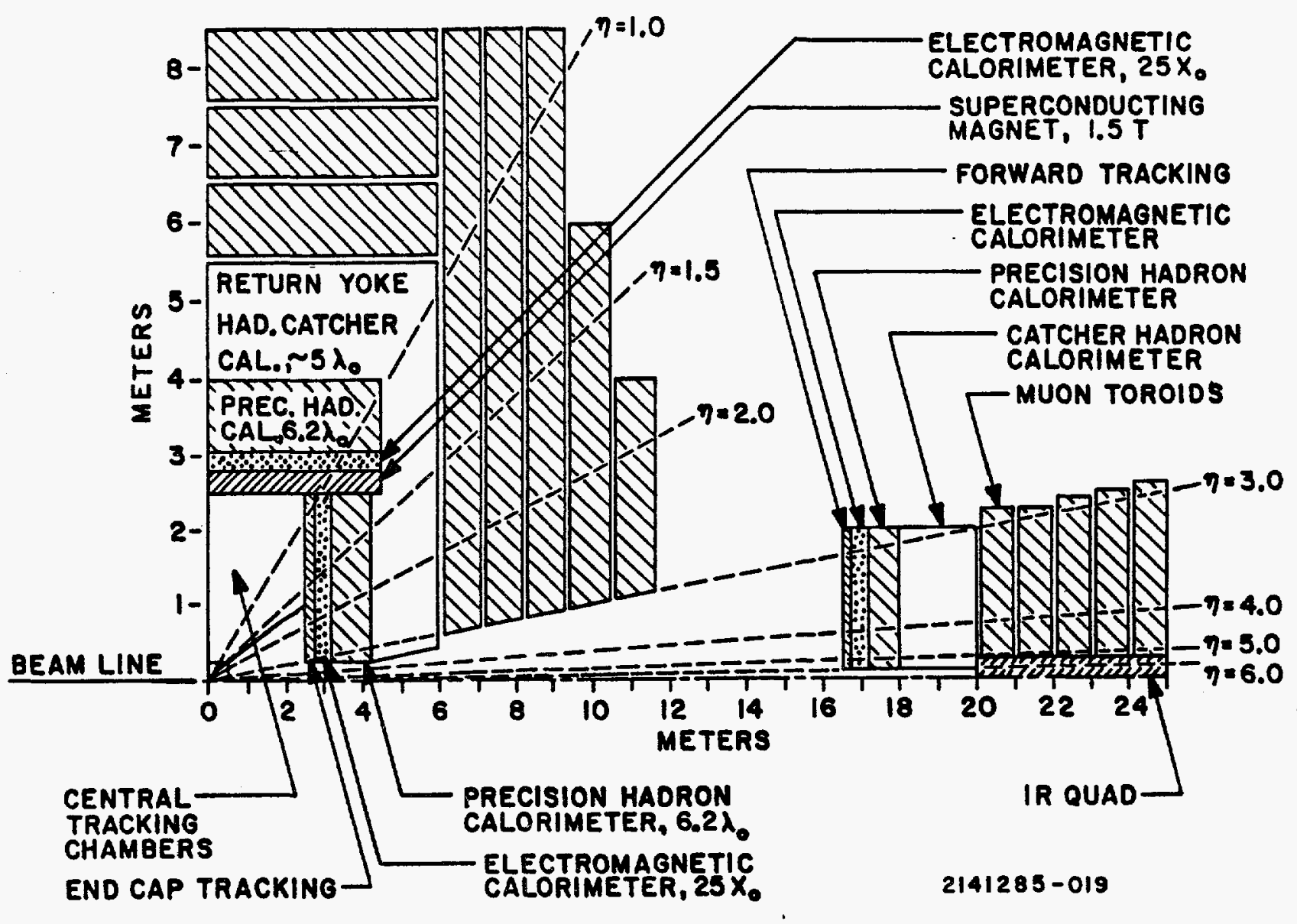

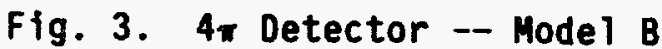


Table 1

$4 \pi$ Detector Dimensions and Parameters

\section{Silicon Vertex Detector}

Rapidity range

Length $(\mathrm{cm})$

No. of layers

Inner radius $(\mathrm{cm})$

Outer radius $(\mathrm{cm})$

No. of strips

Strip width ( $\mu \mathrm{m})$

Resolution ( $\mu \mathrm{m})$

Wire Cham. Vertex Detector

Rapidity range

Length $(\mathrm{cm})$

No. of layers

Inner radius $(\mathrm{cm})$

Outer radius $(\mathrm{cm})$

No. of sense wires

Resolution ( $\mu \mathrm{m}$ )

Central Tracking

Rapidity range

Length $(\mathrm{cm})$

Inner radius

Outer radius

No. of layers

No. of sense wires

No. of strips

Resolution ( $\mu \mathrm{m}$ )

Endcap Tracking

Rapidity range

No. of planes

Inner radius $(\mathrm{cm})$

Outer radius (cm)

No. of sense wires-total

Resolution ( $\mu \mathrm{m}$ )

\section{Forward Tracking}

Rapidity range

No. of planes

Inner radius $(\mathrm{cm})$

Outer radius

No. of sense wires-total

Resolution ( $\mu \mathrm{m}$ )
Model A

-1.5 to 1.5

10 and 20

2

2

5

$18 \mathrm{~K}$

25

10

-1.5 to 1.5

100

25

5

25

$8 \mathrm{~K}$

$\leq 100$

-1.5 to 1.5

100 to 600

25

235

100

$100 \mathrm{~K}$

$32 \mathrm{~K}$

$\leq 175$

1.3 to 3.0

3 each end

50

235

10K

$\leq 200$

3-5

4 each end

20

200

$12 \mathrm{~K}$

$\leq 200$
Mode I B

-1.5 to 1.5

10 and 20

2

2

5

$18 \mathrm{~K}$

25

10

-1.5 to 1.5

100

25

5

25

$8 \mathrm{~K}$

$\leq 100$

-1.2 to 1.2

100 to 500

25

250

100

100K

$32 \mathrm{~K}$

$\leq 175$

1 to 3

3 each end

25

250

10K

$\leq 200$

3-5

4 each end

20

200

$12 K$

$\leq 200$ 
(Table 1 cont'd.)

\section{EM Calorimeter}

Depth in $x_{0}(\lambda)$

Rapidity range

$\Delta y=\Delta \emptyset$

Barre1 $(|y|<1.5-)$

Encap $(|1.5<y<3|)$

Forward $(|3<y<6|)$

Tower size ( $\mathrm{cm} \times \mathrm{cm})$

Barre l (at $y=0$ )

Endcap

Forward

No. of towers

Barrel

Endcap

Forward

Total

No. of strips

No. of long. segs.

Weight (metric tons)

Barre 1

Encap

Forward

Total

\section{Precision Hadron Calorimeter}

U-Liq. Ar

Depth in $\lambda(y=0)$

Rapidity range

$\Delta y=\Delta \phi$

Barrel

Endcap

Forward

Tower size ( $\mathrm{cm} \times \mathrm{cm})$

Barrel $(y=0)$

Encap

Forward

No. of towers

Barre 1

Encap

Forward

Total

No. of long. segments

Weight (metric tons)

Barre?

Endcap

Forward

Total
Model A

$40(1.2)$

-6 to 6

0.02

0.02-0.04

$0.03-0.15$

$5 \times 5$

$2 \times 2$ to $5 \times 5$

$2 \times 2$ to $5 \times 5$

$50 \mathrm{~K}$

$27 \mathrm{~K}$

$14 K$

$85 \mathrm{~K}$

None

3

360

80

35

475

yes

4

-6 to 6

0.04

$0.04-0.08$

$0.06-0.3$

$10 \times 10$

$4 \times 4$ to $10 \times 10$

$4 \times 4$ to $10 \times 10$

$13 K$

$5 K$

$3.5 K$

$21.5 \mathrm{~K}$

1

1700

300

225

2225
Model B

$25(0.8)$

-6 to 6

0.03

$0.03-0.04$

$0.03-0.15$

$8 \times 8$

$2.5 \times 2.5$ to $5.5 \times 5.5$

$2 \times 2$ to $5 \times 5$

$27 K$

$25 K$

$14 K$

$60 K$

$100 \mathrm{~K}$ at shw. max 1

275

65

35

375

yes

6.2

-6 to 6

0.06

$0.06-0.08$

$0.06-0.3$

$18 \times 18$

$3 \times 3$ to $18 \times 18$

$4 \times 4$ to $10 \times 10$

$5 K$

$6 K$

$3.5 K$

$74.5 \mathrm{~K}$

1

3000

600

250

3950 


\section{Catcher Hadron Calerimeter}

Iron-Gas
Depth in $\lambda(y=0)$
Rapidity range
$\Delta y=\Delta \emptyset$
Barrel
Endcap
Forward
Tower Size $(\mathrm{cm} \times \mathrm{cm})$
Barrel ( $\mathrm{y}=0)$
Encap
Forward
No. of towers
Barrel
Endcap
Forward
Total
No. of long. segments
Weight (metric tons)
Barrel
Encap
Forward
Total

Muon System

$\begin{array}{lll}\text { Rapidity coverage } & -5.0 \text { to } 5.0 & -5.0 \text { to } 5.0 \\ \text { Cham. resolution ( } \mu \mathrm{m}) & 200 & 200 \\ \text { Max. drift dist (cm) } & 1 & 1 \\ \text { No. of track layers (3 tube layers per track layer) } & \\ \text { Barrel } & 4 & 4 \\ \text { End } & 4 & 6 \\ \text { Forward } & 6 & 6 \\ \text { No. of wires } & 50 \mathrm{~K} & 50 \mathrm{~K} \\ \text { Barre1 } & 20 \mathrm{~K} & 30 \mathrm{~K} \\ \text { Endcap } & 12 \mathrm{~K} & 12 \mathrm{~K} \\ \text { Forward } & 82 \mathrm{~K} & 87 \mathrm{~K} \\ \text { Total } & 31200 & 16000 \\ \text { Weight (metric tons) } & 5300 & 13500 \\ \text { Barre1 } & 1200 & 1200 \\ \text { End } & 37700 & 30700 \\ \text { Forward } & & \\ \text { Total } & & 1.5 \\ & 1.5 & 900 \\ \text { Fielducting Magnet } & 1200 & 250 \\ \text { Field } & 320 & 1.3 \\ \text { Length (cm) } & 1.7 & 0.3 \\ \text { Radius (cm) } & 0.4 & 30 \\ \text { Thickness } x_{0} & 50 & \\ \text { Thickness } \lambda_{0} & & \end{array}$

\section{Mode 1 A}

yes

8.5

-6 to 6

0.04

$0.04-0.08$

$0.06-0.30$

$15 \times 15$

$6 \times 6$ to $15 \times 15$

$6 \times 6$ to $15 \times 15$

$13 K$

$5 K$

$3.5 K$

$21.5 \mathrm{~K}$

1

7500

1000

300

9700
Model B

yes

5

-6 to 6

0.06

$0.06-0.08$

$0.06-0.3$

$20 \times 20$

$4 \times 4$ to $20 \times 20$

$6 \times 6$ to $15 \times 15$

5K

$6 \mathrm{~K}$

$3.5 \mathrm{~K}$

$14.5 \mathrm{~K}$

1

4000

900

300

5200

\section{Rapidity coverage}

Cham. resolution ( $\mu \mathrm{m})$

Max. drift dist (cm)

No. of track layers Barre 1

End

o. of wires

Barrel

Endcap

Forward

ight (metric tons)

Barre 1

Forward

5300

1200

-5.0 to 5.0

200

-5.0 to 5.0
200

1

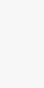


(Table 1 cont'd.)

\author{
No. of Electronics Channels \\ Silicon vertex detector \\ Wire vertex detector \\ Central tracking \\ Endcap tracking \\ Forward tracking \\ EM Calorimeter \\ Prec. Had. Calorimeter \\ Catcher Calorimeter \\ Muon system \\ Tota 1
}

\section{Mode1 A}

integ. readout

16K

min $132 K \max 232 K$

20K

24K

$340-510 K$

$43 K$

$43 K$

$164 K$

$782-1052 K$
Mode I B

integ. readout $16 \mathrm{~K}$

$132-232 K$

20K

$24 K$

220K

29K

29K

$184 K$

654-754K 
Table 2

$4 \pi$ Detector Performance Summary

Parameter

Rapidity coverage

$\Delta p / p$ at $y=0$

$E M \Delta E / E$

$H A D \quad \triangle E / E$

$\Delta p / p$ muons at $1 \mathrm{TeV}$
Model A

$|y|<6$

$0.00023 p(\mathrm{GeV})$

$0.15 / \sqrt{E}$

$0.5 / \sqrt{E}$

0.2
Model B

$|y|<6$

$0.00020 \mathrm{p}$ (GeV)

$0.15 / \sqrt{E}$

$0.5 / \sqrt{E}$

0.2 
luminosity is optimistically assumed in both models. Considerable R\&O effort will probably be required to make this a reality.

\section{Vertex Detection}

The identification of secondary vertices for flavor tagging and sensitivity to new relatively long lived states would be a very useful tool in the $4 \pi$ detector. Three technologies are possible:

(1) high precision wire chambers,

(2) silicon devices,

(3) small diameter glass or plastic fiber devices, or any combination of the three. We have chosen in both models to combine a silicon strip detector with a multi-layer drift chamber. The readout, including filtering, is assumed to be integrated with the detector in each case, vastiy reducing the output data flow. Although there is at present considerable uncertainty in the viability of silicon devices at high luminosities because of radiation damage, such devices have been included in the design in anticipation of a gradual approach to the design luminosity and as yet unexplored radiation hardening techniques. If the technology permits, a pixel device would undoubtedly be even more useful; but, as mentioned earlier, we have considered only small extrapolations of present technology.

The silicon device would be followed by a high resolution wire chamber. Although again radiation damage may limit the operation of such a device, it is sufficiently useful to be included in the designs. Conceptually it might be a rather conventional chamber, but with a high wire density to handle high rates. Maximum drift distances at the inner layer must be $\leq 1 \mathrm{~mm}$. Conservatively the spatial resolution should be $\leq 100 \mu \mathrm{m}$.

\section{Central Tracking}

For both models the central tracking chambers are conventional drift chambers with many wires to handle the high rates and multiplicities, and appropriate cell geometries to handle events overlapping within the lifetime of the chamber. The chambers might be constructed in a few sections although this introduces additional alignment problems. Current division might be implemented on some or all of the wires to provide better "space point" readout. Cathode strips would be interspersed throughout the chamber for more accurate $z$ information. 


\section{Endcap and Forward Tracking}

For both the endcap and forward tracking systems, planar drift chambers would be used. A number of options are possible ranging from radial drift chambers as in COF to straightforward planar chambers, at least for the forward region. The estimates in Table 2 assume a radial geometry.

\section{Electromagnetic Calorimeter}

We have assumed that the electromagnetic calorimetry consists of a uranium (or uranium-copper mix) absorber with liquid argon sampling or noncryogenic liquid sampling if that becomes a reality. The calorimeter is arranged in the usual tower geometry (inside the coil in model $A$ and outside in model B) with the dimensions as indicated in Table 2. In Model A the EM calorimeter is segmented into three longitudinal pieces. In Model $B$ we have chosen to have one longitudinal segment but have inserted $1 \mathrm{~cm}$ strips in orthogonal directions located at shower maximum and spanning many towers to determine more accurately the locations of electromagnetic showers. The optimal configuration needs more study, and we therefore, present a range via Model A and Model B. Typical uranium plate thickness in the EM section might be $3 \mathrm{~mm}$ with a $3 \mathrm{~mm}$ argon gap.

\section{Precision Hadron Calorimeter}

The precision hadron calorimeter inside the coil in Model $A$ and outside in Model B also consists of uranium with liquid argon or noncryogenic liquid readout, and would be in the same physical package as the electromagnetic calorimeter. It has tower sizes which are twice as large as the EM part in each dimension, more suited for hadronic showers.

\section{Catcher Hadron Calorimeter}

Hadron shower energy leaking from the precision hadron calorimeter is measured in an iron-gas sampling calorimeter which also acts as the return yoke of the magnet. The front two-thirds of the calorimeter have $5 \mathrm{~cm}$ iron plates and the last third $10 \mathrm{~cm}$ plates. The tower sizes match the precision hadron calorimeter. The overall thickness in the case of Model $B$ is set by its use as a return yoke.

\section{Muon System}

Muon coverage and independent momentum measurement is provided over $|y|<5.0$ by magnetized iron and drift tube chambers. The tube size is assumed 
to be $\leq 2 \mathrm{~cm}$ (giving a maximum drift distance of $1 \mathrm{~cm}$ ) and there are 3 layers of tubes between each toroid although other arrangements are possible. Model $B$ has more toroids - in the intermediate endcap region than Model $A$.

\section{Electronics}

A summary of the electronics channel count is given in Table 1 . The following assumptions have been made

- Silicon devices have integrated readout on the devices

- The wire vertex detector has current division readout on each wire

- Central track chambers may have current division readout on each wire hence the range in Table 1.

- Endcap and forward tracking chambers have current division on each wire

- Pulse height and timing measurements for the calorimetry are provided by a single readout channel eg. flash ADC's or fast analog shift registers but dynamic range considerations require two such channels per tower element but not for the strips at shower maximum. In the case of Model A the three longitudinal segments in the EM calorimeter might be ganged together for low pulse height readout but separately read out for large pulse heights. This would yield 1.33 channels per segment instead of 2 , giving the range in Table 1 .

A conceptual model of the front end electronics for the calorimetry or tracking would include storage for $\leq 500-1000$ ns until a first level trigger decision was made. Storage could be done by either an analog shift register or by a flash ADC type system. In either case such electronics would no doubt be custom designed. There would of course be differences in speed, precision, etc. between tracking and calorimetry but there are many functional similarities. Much of this electronics would most conveniently be located on the detector.

\section{Triggering and Data Acquisition}

Descriptions of triggering and data acquisition systems for the SSC have been given in the Proceedings of the Snowmass-84 Study, the Large Hadron Collider Workshop and more recently in the Workshop on Triggering, Data Acquisition and Computing for High Energy/High Luminosity Hadron-Hadron Colliders held at Fermilab in November of 1985. It is believed that data acquisition needs may be met by a parallel buss structure using today's typical transfer 
speeds. Substantial filtering via dedicated processors would be required early in the data flow path to reduce event sizes to a reasonable value (0.3-1 megabytes per accepted-event). Permanently recording 1 Mbyte events at a few hert $z$ rate is at or beyond the edge of today's relatively affordable capabilities.

One must assume the development of higher density and/or higher speed recording devices by the mid-1990's to comfortably record data at the few Mbyte/sec rate. Such developments are likely to occur.

The trigger system for either model detector would be the same. One expects to have a multi-level system in which the first level uses analog information from the calorimetry and muon systems to count roughly the numbers of jets, electrons and muons for different transverse energy cuts. The first level trigger would operate in 500-1000 ns. The second level trigger could be analog or digital (or a combination) and would operate within $10 \mu \mathrm{s}$. The final stage of the trigger would be an array of programmable microprocessors, perhaps in a cascade arrangement to give reduction at each step. Estimates indicate that about 1000 VAXI1/780 equivalents might be required. 


\section{Muon Spectrometers Matched to the Full Kinematic Range of the SSC}

\subsection{A High Resolution Muon Spectrometer}

Drawing on the L3 experience, we consider a detector with the following properties:

1. Capability to measure dimuon masses at the level of $1 \%$ for $M=1 \mathrm{TeV}$.

2. Calorimetric capability of measuring over a limited rapidity interval with

$$
\begin{array}{ll}
\Delta E / E \approx \frac{50 \%}{\sqrt{E}} & \text { for hadrons } \\
\Delta E / E \approx \frac{30 \%}{\sqrt{E}} & \text { for electrons and photons }
\end{array}
$$

3. Large magnetic volume for future upgrades.

Figure 4 shows the proposed detector. A large conventional magnet with an inner radius of $8.6 \mathrm{~m}$ and an overall length of $24.9 \mathrm{~m}$ provides a field of 7.5 k6. Three sets of muon chambers provide precision measurements of muon momentum in the bending plane, and standard chambers measure the production angle in the non-bending plane (the $z$-layers). The energy loss of muons through bremsstrahlung and pair production increases quickly with increased muon energy. To correct for this energy loss and to absorb hadrons and track muons, we propose a 284 radiation-length, 9.5 interaction-length active electromagnetic and hadron calorimeter $5 \mathrm{~cm}$ away from the interaction region. This calorimeter consists of the following elements:

1. 20 layers of $3.5 \mathrm{~mm}$ tungsten $+5 \mathrm{~mm}$ proportional tubes

2. 4 layers of $7 \mathrm{~mm}$ tungsten $+5 \mathrm{~mm}$ proportional tubes

3. 128 layers of $7 \mathrm{~mm}$ uranium $+5 \mathrm{~mm}$ proportional tubes

The use of tungsten plates in the first part of the calorimeter system is to shield the uranium from interacting proton beams and backgrounds. The parameters are listed in Table 3.

We discuss these in more detail below.

\section{The Magnet}

We have chosen a design with Al plates which could be produced by industry. 


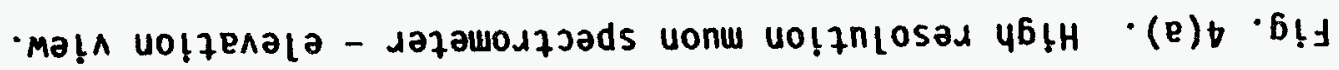

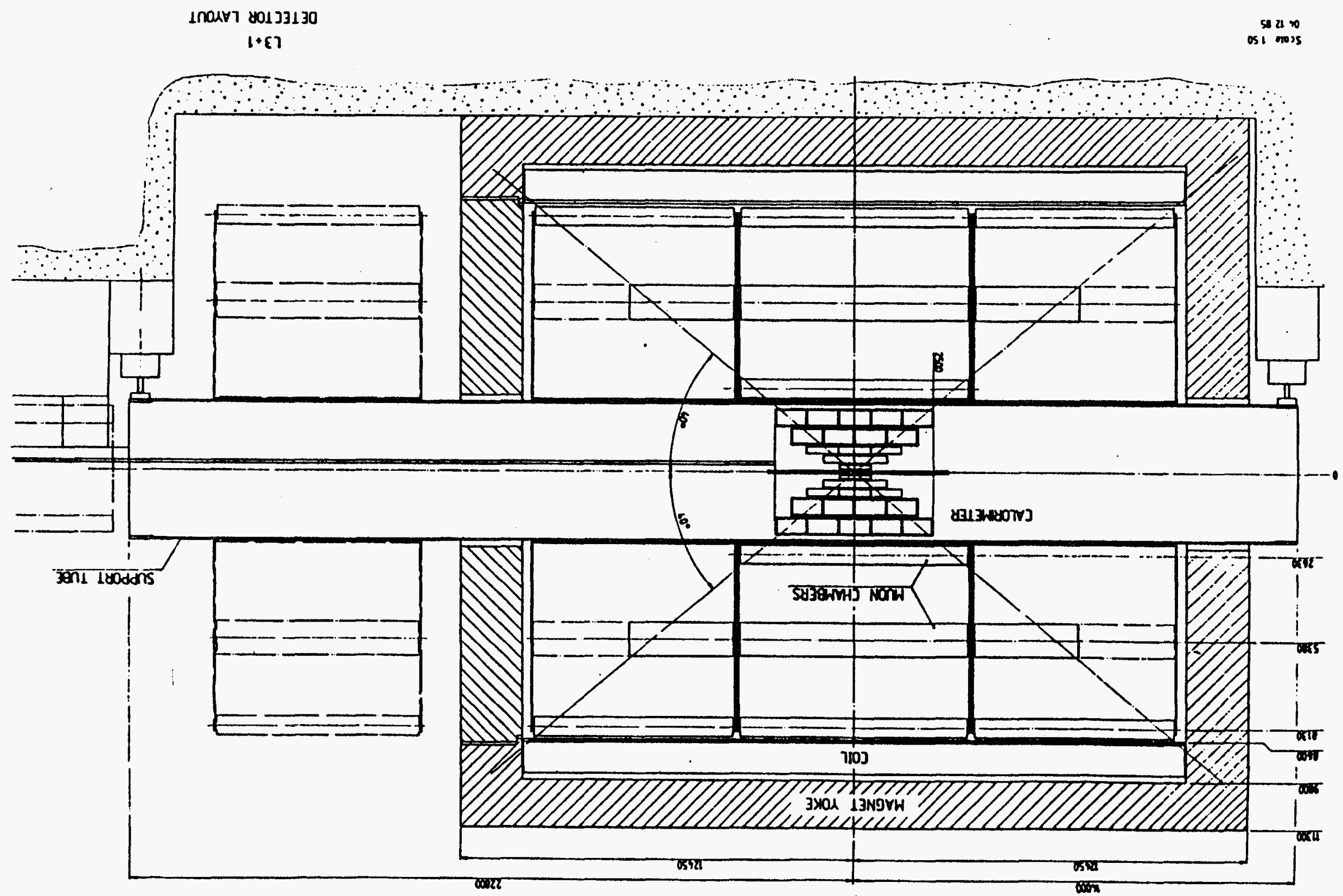




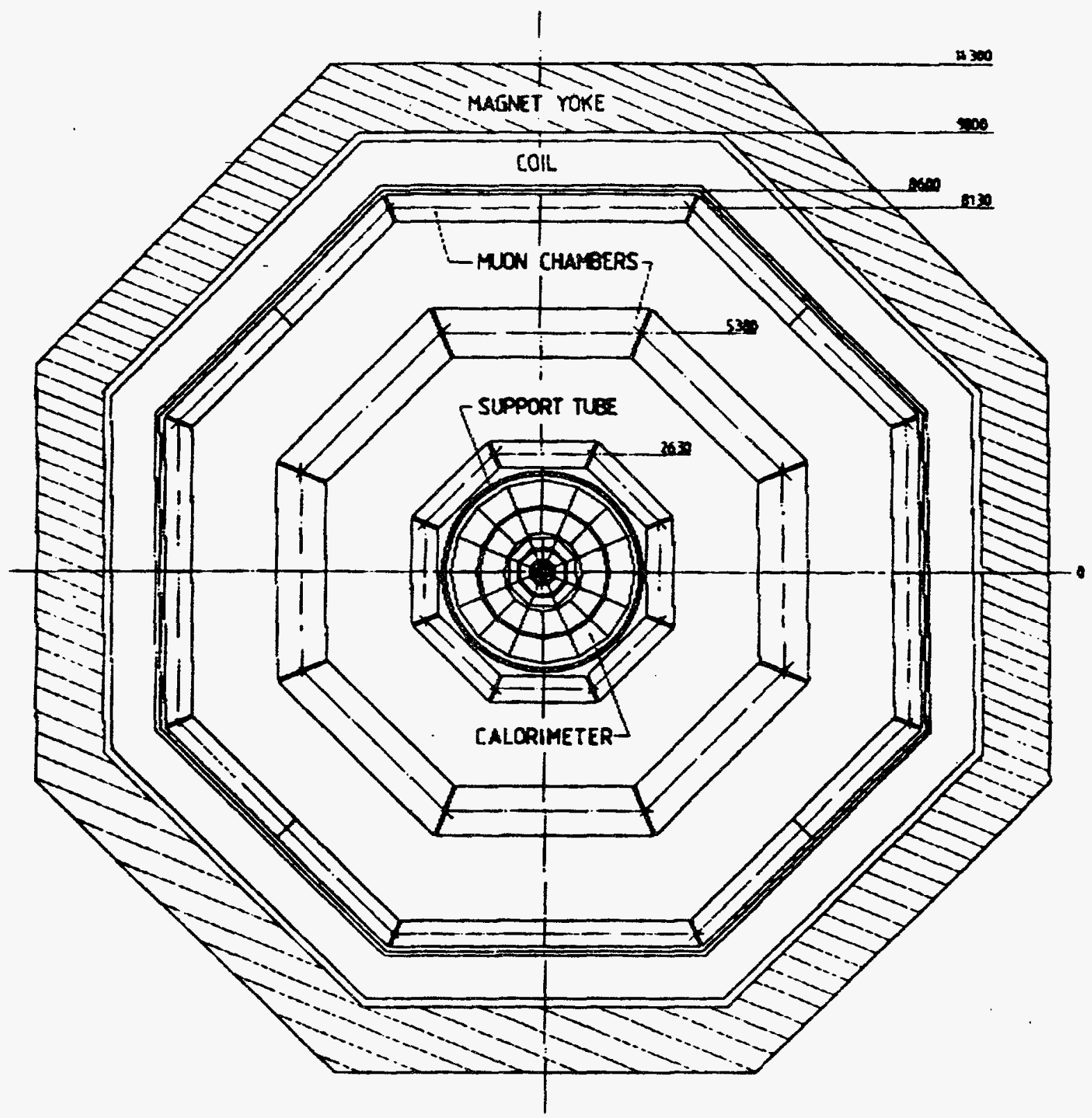

Fig. 4(b). High resolution muon spectrometer end view. 
Table 3

Parameters of Precision Muon Spectrometer

Magnet

Inner radius of $A 1$

$8.6 \mathrm{~m}$

Outer radius of $\mathrm{Al}$

$9.6 \mathrm{~m}$

Inner radius of $\mathrm{Fe}$

$9.8 \mathrm{~m}$

Outer radius of $\mathrm{Fe}$

$11.3 \mathrm{~m}$

Pole thickness

Coil length

$1.95 \mathrm{~m}$

$20.5 \mathrm{~m}$

Al weight

2700 tons

Fe weight

Power consumption

27500 tons

$12 \mathrm{MW}$

EM Calorimeter [Tungsten - Gas]

No. of wires

2000

No. of channels

2000

Radiation lengths

Interaction lengths

20 (20 $1 x_{0}$ layers)

Weight of tungsten

0.7

$450 \mathrm{~kg}$

No. of tungsten plates

160 (3.5 mm thick)

Hadron Calorimeter [Tungsten/Uranium - Gas]

No. of wires

Inner absorber

164000

outer absorber

Interaction lengths

Channels

Weight of tungsten

Weight of uranium

4 layers $-7 \mathrm{~mm}$ tungsten

128 layers - $7 \mathrm{~mm}$ uranium

8.8

10,000

0.9 tons

420 tons

Muon Detector

No. of octants

8

Chamber units

Inner layer - 8

Middle layer - 48

Outer layer - 48

Total area

No. of wires

$1800 \mathrm{~m}^{2}$

Chamber alignment

48000

48 Optical straightness

monitors $(10 \mu \mathrm{m})$

Track calibration

Resolution per wire

16 UV laser systems

$150 \mu \mathrm{m}$ 
The construction is similar to that of L3, i.e., using semi-finished products from industry with a minimum of machining. For these reasons the price of the filling magnetic material should be low.

\section{Muon Detector}

The high resolution detector covers the central region of $80 \%$ of $4 \pi$ solid angle as shown in Fig. 4a. There are three layers of chambers measuring in the bending plane and four double layers of lesser resolution determining the coordinate along the beam. The middle chamber is covered by honeycomb material to minimize multiple scattering. The inner and outer chambers are each enclosed by two double layers of drift chambers measuring the coordinate along the beam. Eight hits in the $z$ chambers are used to detect a track.

For measurements in the bending plane, the three sets of chambers have 32-64-32 sense wire layers respectively. The momentum analys is is done over a length of $5.5 \mathrm{~m}$ and a single layer precision of $150 \mu \mathrm{m}$ is assumed. It is further assumed that a $20 \mu \mathrm{m}$ alignment accuracy can be achieved with appropriate optical monitors.

The sagitta of a $1 \mathrm{TeV}$ muon is $860 \mu \mathrm{m}$, and the measurement precision with $20 \mu \mathrm{m}$ alignment error is about $34 \mu \mathrm{m}$ or $4 \%$. Thus the mass resolution of a 1 TeV dimuon is 1.4\%. The energy losses due to bremsstrahlung and pair production vary from $30 \mathrm{GeV}$ for a $0.5 \mathrm{TeV}$ muon to $286 \mathrm{GeV}$ for a $5 \mathrm{TeV}$ muon, and can be accurately taken into account with the calorimeters.

\section{Hadron Calorimeter}

The calorimeter system consists of two parts:

1. The electromagnetic calorimeter with $I x_{0}$ tungsten plates and

2. the hadronic and electromagnetic parts with $2 x_{0}$ plates of uranium and tungsten.

The outer part of the calorimeter system which measures muon and hadron showers consists of 164,000 proportional $10 \mathrm{~mm} \times 5 \mathrm{~mm}$ tubes interleaved with heavy metal plates. The inner part is $48 \mathrm{~mm}$ thick and uses 4 layers of $7 \mathrm{~mm}$ tungsten plates, whereas the outer part, $1530 \mathrm{~mm}$ thick, requires 128 layers of depleted uranium plates. The electronic readout of the wires is arranged in towers pointing to the vertex in order to measure muons. The structure has an octagonal cross section perpendicular to the beam and its angular coverage starts at $40^{\circ}$ from the beam in both forward and backward directions. A total of 420 tons of uranium and 0.9 tons of tungsten are required. 
The expected total hadron energy resolution is $50 /(E)^{1 / 2} \%$.

\section{Electromagnetic Calorimeter}

This part of the detector consists of 20 layers of tungsten plates $(3.5 \mathrm{~mm}$ thick) interleaved with planes of wire chambers $(5.0 \mathrm{~mm}$ thick) operated in the proportional mode. With this arrangement the calorimeter has 20 radiation lengths and 0.7 interaction lengths.

This calorimeter covers the full azimuthal range and polar angles between $40^{\circ}$ and $140^{\circ}$. The tungsten plates of the calorimeter are mounted concentrically around the beam line. Eight plates of the same dimensions form one octagonally shaped calorimeter layer. The distance between the beam axis and the first tungsten plate is $50 \mathrm{~mm}$. Since the thickness of one layer is $8.5 \mathrm{~mm}$ the entire calorimeter fits within a radial distance between $50 \mathrm{~mm}$ and $220 \mathrm{~mm}$ with respect to the beam line.

With the geometry as described we have 20 different types of tungsten plates with dimensions ranging from $44 \mathrm{~mm}$ by $127 \mathrm{~mm}$ up to $178 \mathrm{~mm}$ by $512 \mathrm{~mm}$.

The expected energy resolution for photons and electrons is

$$
\frac{\Delta E}{E} \approx \frac{30}{\sqrt{E}} \times(E \text { in } \mathrm{GeV}) \text {. }
$$

The Trigger and Data Acquisition System

The high luminosity $\left(10^{33} \mathrm{~cm}^{-2} \mathrm{~s}^{-1}\right)$ and the short time between bunches pose challenging problems for the trigger and data acquisition systems. To minimize the dead time, the system should be configured with several levels of event buffering and several levels of triggers. The event rate should be reduced successively by going through each level of trigger. The null data suppression must be done at the very front end and the event size may preferably be reduced at each level of the triggering by the use of local and distributed intelligence.

The level 1 trigger will perform:

1. Track finding in the muon chamber

2. Calculation of the total energy

3. Calculation of the transverse energy and missing transverse energy

4. Search for the energy cluster pointing back to the vertex

Each of these triggers as well as the combination of them will cover most of the physics objectives of the proposed detector. A complete digital leve1-1 trigger is favored over the analog method due to the merit of the 
reliability and the reproducibility. The level-1 trigger must have a bandwidth of $30 \mathrm{MHz}$. The decision time may be about 300 ns and the pipelining of about 30 events is necessary. The data digitizer of the calorimeter and the muon chamber must have the same size as the event buffer to ensure the deadtime free operation of the level-1 trigger. The calorimeter ADC must have either analog (CCO) or digital (Flash $A D C+$ fast memory) event storage with the clock speed of $30 \mathrm{MHz}$ and 30 events deep.

The pipeline TOC of the muon chamber has to have an additional pipeline length of $300 \mathrm{~ns}$ in addition to its inherent drift time of $1 \mu \mathrm{s}$. A positive decision of the level-1 trigger starts the conversion and/or buffering of data into the next level of the event memory. This may take $300 \mu \mathrm{s}$ for completion. The level-2 trigger may be formed within $100 \mu \mathrm{s}$ and a negative level-2 result will abort this data buffering thus reducing the system deadtime. The leve1-1 trigger will be performed by the hardwired processors with little programability. The level-2 must be more flexible to allow an elaborate correlation between parameters produced by the level-1 triggers.

The leve 1-3 and level-4 triggers will be a bank of programmable processors being deployed in a round robin fashion to supply a necessary throughput. The level-3 processor may be a fast pipelined processor and the leve 1-4 processor may be an IBM emulator or a fast microprocessor embedded in the FASTBUS system. The combination of the level-3 and level-4 triggers has to reduce the event rate down to $5 \mathrm{~Hz}$, which can be conveyed to the host computer and stored in a mass storage. The main event builder to build a complete event and send it to the level-4 trigger must have the highest bandwidth possible. This is to ensure that the most flexible level-4 trigger can be readily expanded to cope with the higher trigger rate. For this reason, it is desirable to have several event builders working in parallel to supply the data for several separate banks of the leve1-4 processors. The preprocessing of the data into the form easily usable by the level-4 processor will also be useful to have a higher throughput of the level-4 trigger. This may be performed in the lower level event builders.

The leve1-1 and level-2 triggers will be working on the dedicated trigger signals which supply a small amount of data in a short time. The level-3 trigger may be working both on the trigger data and a part of the main data. The level-4 trigger will have access to all of the data. 


\subsection{An Iron Muon Spectrometer}

The design goals of this spectrometer are

- detection and measurement of muon momenta over the rapidity range $|y|<5$

- measurement of muon momenta up to the kinematic limit at the SSC (about $5 \mathrm{TeV}$ ) for at least isolated muons

- some detection of muons in coincidence with missing transverse energy or electrons

- operation at the design luminosity of the $\operatorname{SSC}\left(10^{33} \mathrm{~cm}^{-2} \mathrm{sec}^{-1}\right)$

The spectrometer is shown in Fig. 5, and the spectrometer parameters are given in Table 4 .

The detection of muons in the few hundred $\mathrm{GeV}$ to $\mathrm{TeV}$ range is qualitatively different from detection in the few GeV range required in present colliding beam experiments. Pair production, bremsstrahlung, and nuclear interactions dominate muon energy loss above about $300 \mathrm{GeV}$ in iron. Electromagnetic showers from such energy loss will tend to occasionally obscure the muon track in the iron. For this reason more tracking redundancy is needed at high energies, with iron segments of about $50 \mathrm{~cm}$ thickness. The average energy loss is negligible compared to the intrinsic resolution of an iron spectrometer which is limited by multiple scattering to be worse than about $10 \%$ at all energies. While an occasional muon will suffer a catastrophic energy 1oss, the fluctuations in energy loss may almost always be ignored in an iron spectrometer.

\section{Inner Wire Tracking Chambers}

Since the magnetic field in the iron in the barrel region of the detector is such as to give bending in the polar angle direction, the inner tracking chambers must measure this angle precisely. The wires are therefore transverse to the beam in the barrel region. Operation at a luminosity of $10^{33} \mathrm{~cm}^{-2} \mathrm{~s}^{-1}$ will likely require small drift distances to avoid radiation damage and pile-up. The chambers start $50 \mathrm{~cm}$ from the interaction point with $3 \mathrm{~mm}$ wire spacing. The wire spacing grows to $6 \mathrm{~mm}$ at $1 \mathrm{~m}$ radius. In the channel count given in Table 4 , it is assumed that $25 \%$ of the wires will be read out from both ends for current division. 


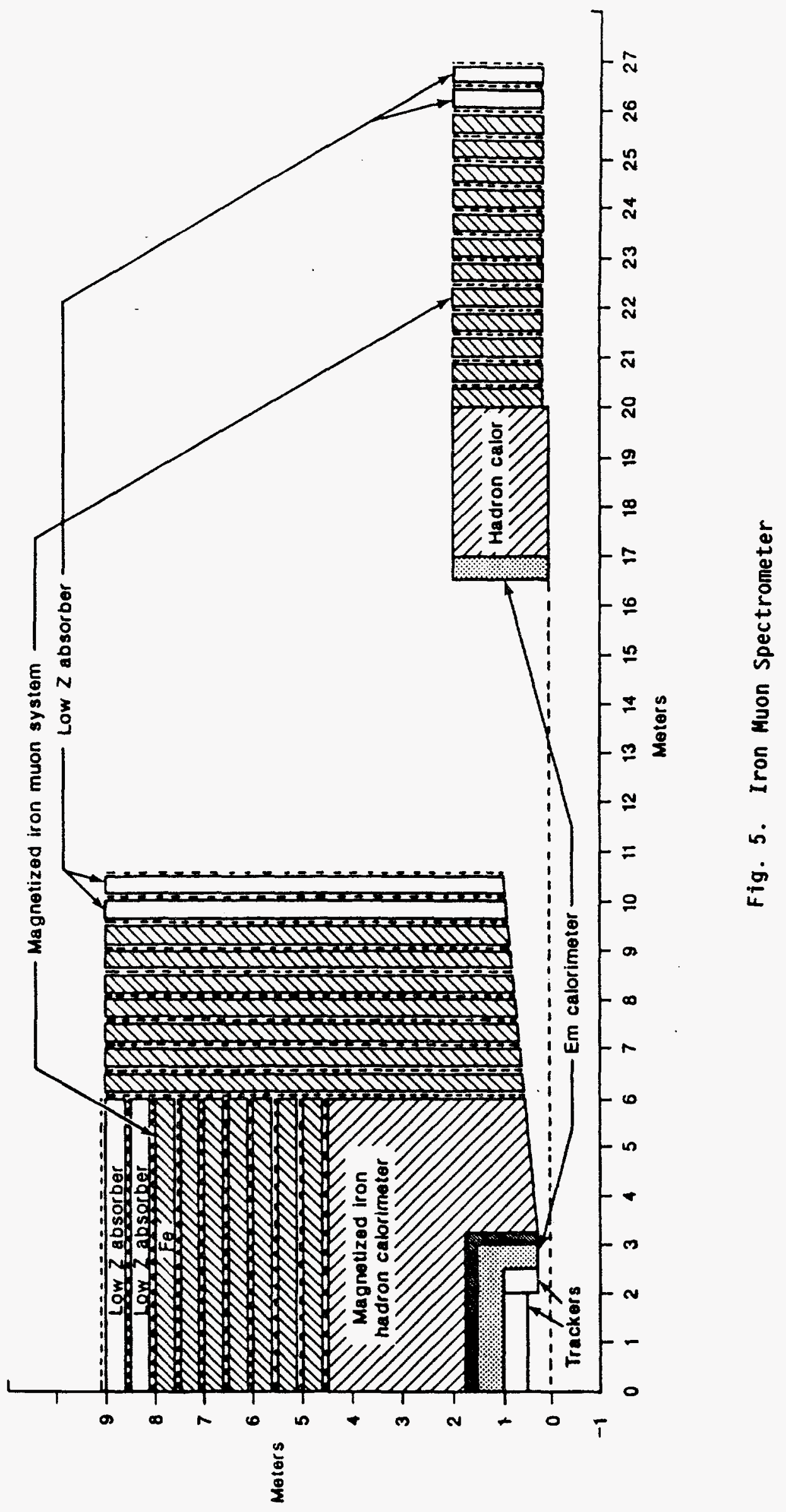


Table 4

Parameters of an Iron Muon Spectrometer

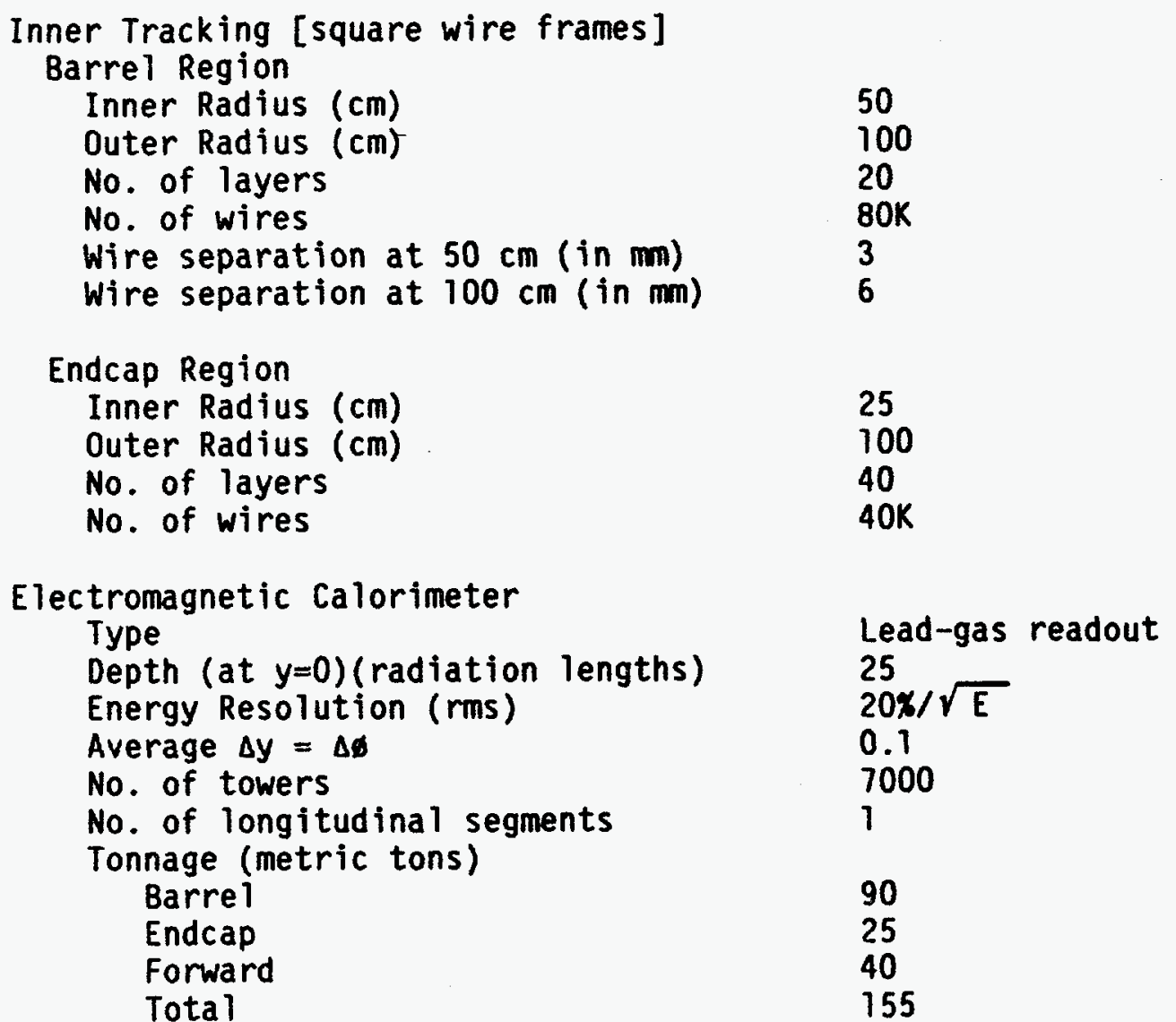

Hadron Calorimeter

Type

Depth (at $y=0$ ) (interaction lengths)

Energy Resolution (rms)

Average $\Delta y=\Delta \sigma$

No. of towers

No. of longitudinal segments

Tonnage (metric tons)

Barrel

Endcap

Forward 475

$\begin{array}{ll}\text { Forward } & 475 \\ \text { Total } & 6275\end{array}$

Muon Iron

Magnetic Field (T)

2

Tonnage (metric tons)

Barre 1

Endcap

Forward

Iron-gas readout

12

$100 \% / \sqrt{E}$

0.2

1700

2

2750

3050

Total

16500

14000

1200

31700 
Muon Tracking in Hadron Calorimeter Chamber Resolution ( $\mathrm{m}$ )

No. of layers

Separation between layers $(\mathrm{cm})$

No. of tube layers/tracking layer Barrel

Endcap

Forward

Areas (sq. meters) $\times$ layers

Barrel

Endcap (both sides)

Forward (both sides)

Total

No. of Tubes

Barre 1

Endcap

Forward

300

6

50

Tota 1

1

2

2

900

2000

400

3300

7500

11000

5000

23500

Muon Tracking in Muon Iron

Chamber Resolution ( $\mu \mathrm{m})$

No. of layers

Separation between layers (cm)

No. of tube layers/track layer

Barrel

Endcap

Forward

Areas (sq. meters) $\times$ layers

Barre 1

Endcap

Forward

Tota 1

No. of Tubes

Barrel

Endcap

Forward

Total

300

10

50

1

2

2

6500

10200

800

17500

54000

36000

12000

102000

Trigger Scintillator

Area (sq. meters)

Barrel

Endcap

7500

500

Forward

-none-

Electronics channels

Inner Tracking

Electromagnetic Calorimeter

Hadron Calorimeter

Muon Tracking

Scintillator

Total

$150 \mathrm{~K}$

$14 K$

$3.4 K$

$251 \mathrm{~K}$

$2 K$

$420 \mathrm{~K}$ 


\section{Electromagnetic and Hadronic Calorimetry}

Since the focus of this detector is primarily on muon detection, the calorimetry has not been optimized. The goals of the calorimetry are to

- provide triggering capability beyond an inclusive muon trigger, e.g., missing energy or jet energy measurements

- provide hermiticity for missing energy measurements in coincidence with a muon or muons

- provide some (isolated) electron identification

- provide some jet energy measurements

The electromagnetic calorimetry is provided by a 25 radiation length deep device with a lead radiator and gas sampling. A simple tower structure is used with no longitudinal segmentation. It may be necessary to operate at low gas gain to avoid radiation damage and pileup of soft photons and charged particles.

The hadron calorimeter is a magnetized iron gas sampling calorimeter. Again a tower structure is used with two longitudinal segments. Muon tracking tubes/chambers are placed every $50 \mathrm{~cm}$ in addition to the tower readout. Plate thickness varies from about $10 \mathrm{~cm}$ in the front of the calorimeter to about $25 \mathrm{~cm}$ at the rear.

\section{Muon Iron and Tracking}

Outside of the hadron calorimeter, muon tubes/chambers are located every $50 \mathrm{~cm}$ between magnetized iron. In the barrel region there is only one layer of tubes every $50 \mathrm{~cm}$. In this region the iron is magnetized in a toroid fashion around the beam so that the magnetic deflection is in the "theta direction." The tubes/chambers would then be oriented such that the drift distance measured the deflection. The other coordinate would come from current division. In calculating the number of muon tracking channels, we assume that simple tube chambers with a $1 \mathrm{~cm}$ maximum drift are used. At the outside of the iron the chambers are located to provide a lever arm of about 1 $m$. It may be useful to place low $Z$ absorber between these chambers, as shown in Fig. 5, to absorb soft showers produced in the last section of iron.

In the endcap and forward region circular toroids would be used. In this region there are two-crossed-tube/chamber layers every $50 \mathrm{~cm}$ to measure the deflection. At least two (more would be better) such layers are needed to measure the deflection adequately. 
Also located in the muon iron is one layer of scintillator for fast triggering and timing information.

\section{Electronics}

The following assumptions have been made about the electronics in order to obtain the numbers given in Table 4.

- the inner tracking chambers have current division on $25 \%$ of the wires

- only time information is required for the other $75 \%$

- the muon tubes all have current division and would also require at least double hit if not full waveform sampling to reduce electromagnetic shower background.

- each tower element is assumed to require two channels for dynamic range considerations. This may not be necessary if the gain is reduced.

- each scintillation counter is assumed to have an area of 1 sq. meter 


\section{Upgrade 4\% Detector with a Forward Spectrometer}

\subsection{Forward Spectrometer}

It will be desirable at the SSC to explore physics at values of Feynman-x greater than those accessible to a large central detector but smaller than the "Roman pot" style detectors can observe. We therefore propose a forward spectrometer on one side of the IR.

The spectrometer, described here and shown in Fig. 6, consists of a forward detector 100 meters downstream of the collision point and an intermediate detector at 35 meters. It provides good segmentation in tracking and calori-metry for rapidities from 3.5 to 8.0. A central detector derived from the upgrade of an existing detector can be a suitable match to the forward spec-trometer. The two detectors would be able to supplement each other's capa-bilities, especially in the determination of missing energy.

With such a spectrometer, the production dynamics and properties of "low mass" states -- in the range of 100 to $500 \mathrm{GeV}$-- can be effectively studied. This range includes the $W$ and $Z$ bosons, of which there should be copious forward production. The detector would have a special advantage for any new states which are diffractively produced, including intrinsic heavy quarks. In addition, particle identification may be practical in the forward direction, and vertex detection may be accomplished.

Mass resolutions for objects like new Z's which decay into electrons will, with modest segmentation, be limited by systematic uncertainty at the level of about 1\%. The di-jet invariant mass resolution should be at least as good as, and perhaps somewhat better than, that achieved by a large central detector. The detector should also do well at studying multi-jet topologies.

Because of the high rate of events expected in this mass region at $\sqrt{s}=40 \mathrm{TeV}$, the detector could explore much of the relevant physics at a luminosity of about $10^{32} \mathrm{~cm}^{-2} \mathrm{sec}^{-1}$, or about $10^{7}$ interactions per second. The long distance to the first quadrupoles limits the luminosity to about a factor of ten below the luminosity at the low-beta interaction regions. The modest luminosity would be compatible with an upgrade central detector using current technology, equipped with state-of-the-art vertex detectors close to the beam. 


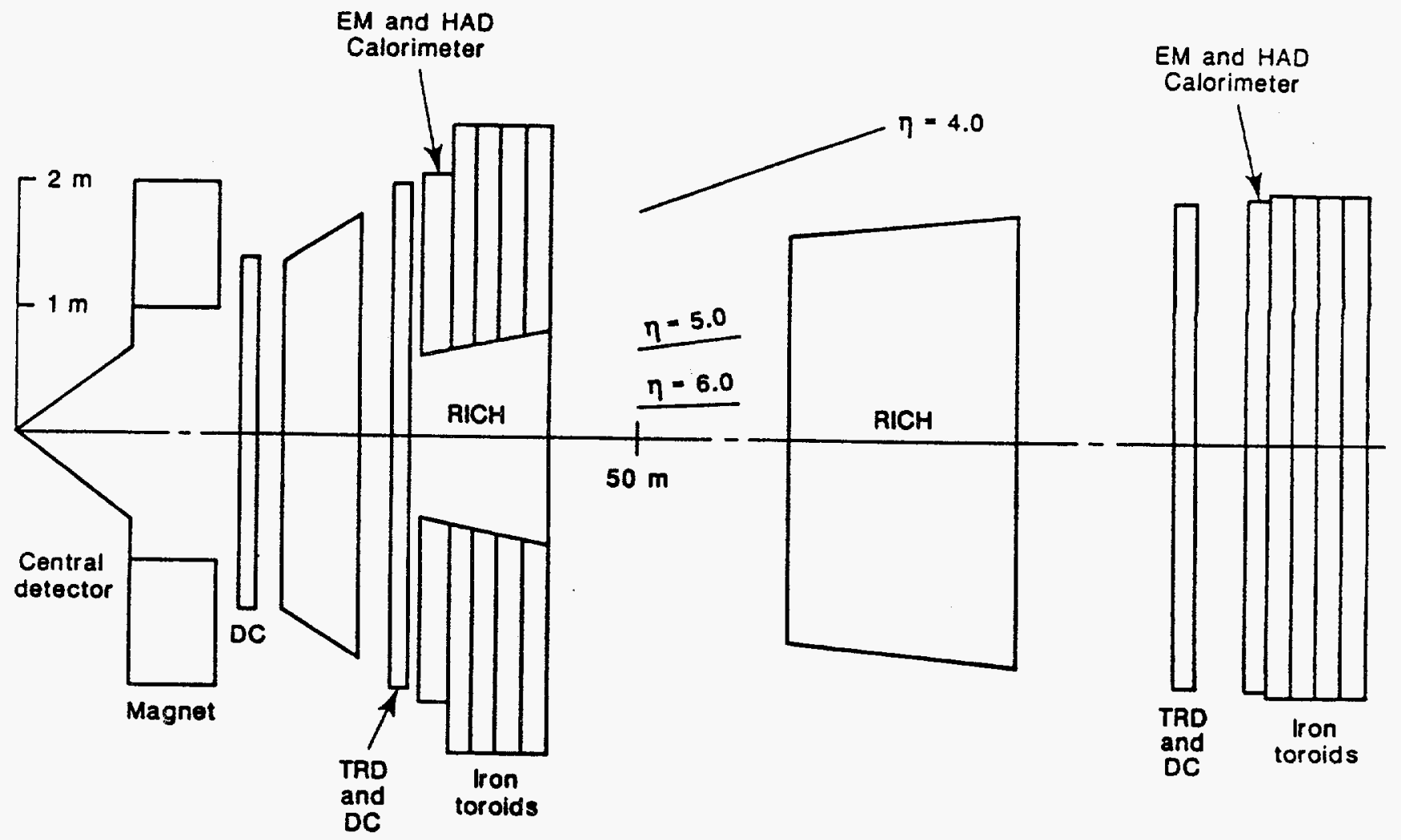

Fig. 6. Forward Spectrometer Elements 
The detailed parameters of the forward spectrometer are given in Table 5 . A description of various parts of the device is given below.

The forward detector, located 100 meters downstream, covers angles below 20 mrad, with a sufficiently large hole to allow the beams to pass. Good resolution and segmentation extend down to about $20 \mathrm{~cm}$ from the beam, although coarser coverage continues down to $5 \mathrm{~cm}$. The electromagnetic section of the calorimeter consists of 35 radiation lengths of lead-liquid argon, followed by a hadronic section of 6 absorption lengths and finally a magnetized iron catcher and muon toroid. The electromagnetic section has towers $1.5 \mathrm{~cm}$ square out to a distance of $75 \mathrm{~cm}$ from the beam, with $4.0 \mathrm{~cm}$ squares from 75 to 200 $\mathrm{cm}$. This gives lateral segmentation ( $\Delta n$ by $\Delta \emptyset$ ) of 0.02 to 0.05 from $20 \mathrm{mrad}$ down to $3 \mathrm{mrad}$, degrading to 0.10 at $1.5 \mathrm{mrad}$. There are a total of 20,000 towers, each with three layers of longitudinal segmentation. The hadronic towers are twice as large in each dimension, for a total to 5,000 towers in the hadronic section of the liquid argon detector, and another 5,000 in the iron catcher. Thus, there are a total of 70,000 channels of calorimetry.

In front of the calorimeter is a tracking section with 4 modules, each with 5 layers of drift chambers followed by a transition radiation detector (TRO). The purpose of the TRD's is to improve the electron/hadron separation in the momentum range $2-200 \mathrm{GeV} / \mathrm{c}$. The segmentation of the TRD is matched to that of the hadronic calorimeter, with 5,000 pads in each module for a total of 20,000 channels. The charged multiplicity in the angle range 2-20 mrad is expected to be about 20 , and the occupancy rate is acceptable except very close to the beam to the beam at angles below the nominal acceptance of the spectrometer.

The iron toroid system consists of 8 meters of magnetized iron downstream of the liquid argon calorimetry. The first section of $1.5 \mathrm{~m}$ is the catcher for the hadron calorimeter. The remaining iron has 4 modules of chambers, each capable of measuring the muon angle. The muon system could be identical to that described for the forward region in the 01 detector of the 1984 Snowmass study. Below a momentum of about $1 \mathrm{TeV} / \mathrm{c}$, the momentum resolution is limited by multiple scattering to about $15 \%$.

In the angular acceptance of this detector, most of the hadrons from heavy quark decays are in the momentum range 20-500 GeV/C. There is sufficient space along the beam for a ring-imaging Cherenkov counter (RICH) which identifies hadrons over most of this range. Good $\pi / K / p$ separation offers a large 
Table 5

Parameters for Forward Detector

Forward Detector

General

Maximum distance from interaction

$110 \mathrm{~m}$

Transverse size

$4 \mathrm{~m} \times 4 \mathrm{~m}$

Accepted angle

$0.5-20.0 \mathrm{mrad}$

Rapidity

4.6-8.0

Electromagnetic Calorimeter

Depth

$35 \mathrm{X}_{0}$ of $\mathrm{Pb}-\mathrm{LA}$

$\Delta \eta=\Delta \phi$

Cell size

$0.02-0.05$ ( $\theta>3 \mathrm{mrad})$

$1.5 \mathrm{~cm}(\theta<7.5 \mathrm{mrad})$

$4.0 \mathrm{~cm}(\theta>7.5 \mathrm{mrad})$

No. of towers

20,000

No. of longitudinal segments

3

Mass

35 tons

Hadron Calorimeter

Depth

$\Delta n=\Delta \emptyset$

Cell size

$6 \lambda$ of $\mathrm{Pb}-\mathrm{LA}$

$0.04-0.10$

$3.0 \mathrm{~cm}(\theta<7.5 \mathrm{mrad})$

No. of towers

No. of longitudinal segments

$8.0 \mathrm{~cm}(\theta>7.5 \mathrm{mrad})$

5,000

Mass

1

Depth of hadron catcher

No. of towers in catcher

200 tons

$8 \lambda$ of $\mathrm{Fe}$

5,000

Muon Toroids

Depth of magnetized iron

No. of measuring planes

$\Delta p / p$

No. of wires (with charge division)

Mass (including catcher section)

$8 \mathrm{~m}$ (including catcher)

20

$15 \%$ of $p<1 \mathrm{TeV} / \mathrm{c}$

20,000

1,000 tons

Transition Radiation Detectors - Drift Chambers

No. of TRD measuring layers

4

No. of TRD pads per layers

5,000

No. of DC measuring planes

20

Resolution

$200 \mu \mathrm{m}$

No. of DC sense wires

40,000

Ring Imaging Cherenkov Counter

Gas

Index of refraction

Length of Radiator

$\pi / K$ separation

$K / p$ separation

No. of photon detectors

No. of channels

$1 \mathrm{~atm} \mathrm{He}$

$1+3.5 \times 10^{-5}$

$18 \mathrm{~m}$

$20-200 \mathrm{GeV} / \mathrm{C}$

$70-400 \mathrm{GeV} / \mathrm{c}$

32

25,000 
Magnet

Aperture

Length

Field
$2 m \times 2 m$

$7 \mathrm{~m}$

0.7 Tesla

Total No. of Channels (forward detector)

Calorimeters

TRD's

Drift Chambers

RICH

Muon Chambers

70,000

20,000

40,000

25,000

20,000

\section{Intermediate Detector}

\section{General}

Transverse size

Accepted angle

Rapidity acceptance of calorimeter

Electromagnetic Calorimeter

Depth

$\Delta \eta=\Delta \emptyset$

Cell size

No. of towers

No. of longitudinal segments

Mass

Hadron Calorimeter

Depth

$\Delta \eta=\Delta \emptyset$

Cell size

No. of towers

No. of longitudinal segments

Mass

Depth of hadron catcher

No. of towers in catcher

Muon Toroids

Depth of magnetized iron

No. of measuring plane

$\Delta p / p$

No. of wires (with charge division)

Mass (including catcher section)

Transition Radiation Detectors

No. of TRD measuring layers

No. of pads/layer
$4 m \times 4 m$

20-60 mrad

$3.5-4.6$

$35 \mathrm{X}_{0}$ of $\mathrm{Pb}-\mathrm{LA}$

$0.02-0.06$

$4.0 \mathrm{~cm}$

10,000

3

30 tons

$6 \lambda$ of $\mathrm{Pb}-\mathrm{LA}$

$0.04-0.10$

$8.0 \mathrm{~cm}$

2500

1

175 tons

$8 \lambda$ of $\mathrm{Fe}$

2500

8m (including catcher)

20

$15 \%$

10,000

800 tons

4

2500 
Ring Imaging Cherenkov Counter

Gas

Index of Refraction

Length of Radiator

$\pi / K$ separation

$K / p$ separation

No. of photon detectors

No. of channels

Orift Chamber Planes

No. of DC planes

No. of sense wires

Silicon Vertex Detector

No. of planes

Transverse size

Strip width

Multiple scattering contribution

to error on impact parameter

Total No. of Channels (intermediate detector)

Calorimeter

TRD's

Drift Chambers

RICH

Muon Chambers

Vertex Detector
He-N2 mixture

$1+1.0 \times 10^{-4}$

7 meters

10-100 GeV

35-200 GeV

32

25,000

10 at 20 meters

10 at 30 meters

40,000

4 each at $z=1.5,3,6,10 \mathrm{~m}$

$10 \mathrm{~cm}$ full width

$20 \mu \mathrm{m}$

$40 \mu \mathrm{m} / \mathrm{P}_{\mathrm{T}}$

35,000

10,000

40,000

25,000

10,000

64,000 
advantage over the central detectors for studying $D, B$, and $T$ decays. The active area of the RICH, extending from about 20 to $200 \mathrm{~cm}$ from the beam pipe, is covered by 32 mirrors each focusing to a $25 \mathrm{~cm} \times 25 \mathrm{~cm}$ photon detector. Each detector sees about one Cherenkov ring. A photon detector suitable for high rates is the multistep avalanche chamber used by Fermilab E-605. Anode strips with $1 \mathrm{~mm}$ spacing give adequate resolution, although pads could be used in one plane.

To obtain Cherenkov identification and good momentum resolution, a magnetic field is required. There is space between the central detector and the intermediate-angle detector for a substantial dipole. The aperture of the magnet must be about 2 meters in each dimension. The effect of the magnet on the proton beam is a serious problem. Possible solutions are to exclude the field from the beam pipe, to use a quadrupole field, or to use a dipole field which compensates a dipole field in the central detector. Although designing such a magnet would take further effort, it seems likely that a reasonable solution could be found.

The momentum resolution of this detector is remarkable good. As an example, a dipole field situated at $14 \mathrm{~m}$ from the I.P. providing a $\mathrm{p}_{t} \mathrm{kick}$ of $2 \mathrm{GeV}$ and chamber stations at $20 \mathrm{~m}, 30 \mathrm{~m}$ and $100 \mathrm{~m}$ each with $150 \mu \mathrm{m}$ resolution should yield a $\Delta p / p$ better than $1 \%$ up to about $1 \mathrm{TeV}$. Below about 200 $\mathrm{GeV}$, the resolution is dominated by multiple scattering in the spectrometer itself which must employ large helium bags.

This performance is based upon the assumption that the primary vertex is given accurately by the central detector. For objects (like B's and D's) which decay away from th primary vertex, the silicon strip detectors (discussed below) in the forward direction allow the recognition of displaced vertices at the expense, however, of somewhat degraded mass resolution due to multiple scattering. Nevertheless, over most of the kinematic range of this device, $\triangle M / M$ should be less than $1 \%$ even for objects as light as the $0^{0}$.

The intermediate detector is very similar to the forward detector, but located only $35 \mathrm{~m}$ from the interaction point, and with a $1.5 \mathrm{~m}$ square hole in the middle. This section covers angles from 20 to $60 \mathrm{mrad}$, or 3.5-4.5 units of rapidity. The segmentations of the calorimetry and TRO's are identical to those in the outer region of the forward detector, with each $4 \mathrm{~cm}$ electromagnetic tower covering $\Delta \eta$ and $\Delta$ of 0.02-0.06 units. The numbers of channels 
are about a factor of two less than for the forward detector. It is important to minimize the sharing of showers at the inner edge of the calorimeter. Thus, a small part of the calorimeter might be made of the densest possible construction. The tracking chambers would be active inside the hole in the calorimeters, providing a first stage of tracking for the forward detector. The RICH is similar to that for the forward detector, but operating at a threshold which is lower by about a factor of 2 . It will not be active in the central region, where the tracks are very dense and the momenta are too high.

Another feature which allows the study of heavy quark physics is high resolution vertex detection. Silicon strip detectors extending from 2-5 cm radius can be placed at a few points along the beam axis. The full vertex resolution of about 10 microns is achieved for $p_{T}>3 \mathrm{GeV} / \mathrm{c}$ independent of angle. The lower luminosity required by the high beta interaction region allows operation of strip detectors at this distance. The practical problems of mounting and alignment of the detectors are very similar to those faced in fixed target experiments, and are somewhat less than for the barrel vertex detector. The multiplicity seen by each module of strip detectors is rather modest, as they subtend only about one unit of rapidity each.

\subsection{Detector Upgrades}

Our model for costing the initial complement of SSC detectors includes an upgraded, existing detector intended to operate at about $\mathscr{L}=10^{32} \mathrm{~cm}^{-2} \mathrm{~s}^{-1}$. Although less ambitious than a new large $(\sim 4 \pi)$ detector optimized for the SSC physics, the upgraded detector should provide a complementary view of a broad range of new physics topics. This upgraded detector in our model will also supplement the special-purpose spectrometer described in 3.1. It may, in addition, provide specific detection capabilities which are not represented in the new $4 \pi$ detector--either because of the rigors of the $\mathscr{L}=10^{33} \mathrm{~cm}^{-2} \mathrm{~s}-1$ environment or because of the particular optimization choices made in that detector. Examples of such specific features might include high precision, close-in microvertex detectors, electron-identifying transition radiation detectors, or a dipole field shape which makes the forward angles more accessible to momentum measurements. Finally one might hope that in the spirit of the upgrade the detector would be ready for physics very quickly after SSC turn-on. 
As discussed in section III, we have considered upgrades of CDF, $D \varnothing$, and UA1, and discuss all three in this report. The organization of effort within the Panel has been-such that the details of the upgrades, as well as those for the forward spectrometer have been largely done by independent-subgroups. We have not taken the time to make a careful match of the upgraded detector to the forward spectrometer with respect to solid angle coverage. We believe that the cost differentials involved in these fine adjustments are too small in relation to overall uncertainties to warrant more effort in that direction.

\subsubsection{Upgraded CDF Detector}

The COF detector is a "general purpose" instrument with good capabilities for identification of electrons, muons, missing transverse momentum, and jets over a large solid angle.

It was designed to cover \pm 4 units of rapidity and will provide the same coverage at $20 \mathrm{TeV}$. The additional features which need to be provided in an upgrade are (1) much greater segmentation in both the tracking and the calorimetry due to the higher multiplicities, (2) thicker calorimetry to allow containment of particles of nearly ten times higher energies, and (3) the capability to operate at interaction rates approximately 100 times higher than those for which the detector was initially intended to operate. Figure 7 shows the existing and added components. The improvements proposed are discussed below. The corresponding parameters are listed in Table 6 . Performance characteristics are listed in Table 7.

\section{Calorimetry}

It is relatively simple to conclude that nearly all of the present sampling media in the calorimeters will be discarded. Even if the characteristics of the events were not to change, it is unlikely that either the scintillator or the gas chambers are sufficiently robust that one would think of using them in a new experimental program beginning ten years from now. The requirements of better segmentation and much higher event rates make this conclusion inescapable. It is desirable, however, to salvage as much of the mechanical construction as is possible. We assume, at a minimum, that the yoke and coil are kept largely intact. Similarly, much of the steel work in the existing calorimeter can probably be used.

Mechanical Construction. It appears feasible to thicken the end wall, end plug, and forward hadron calorimeters by adding to the downstream end of 


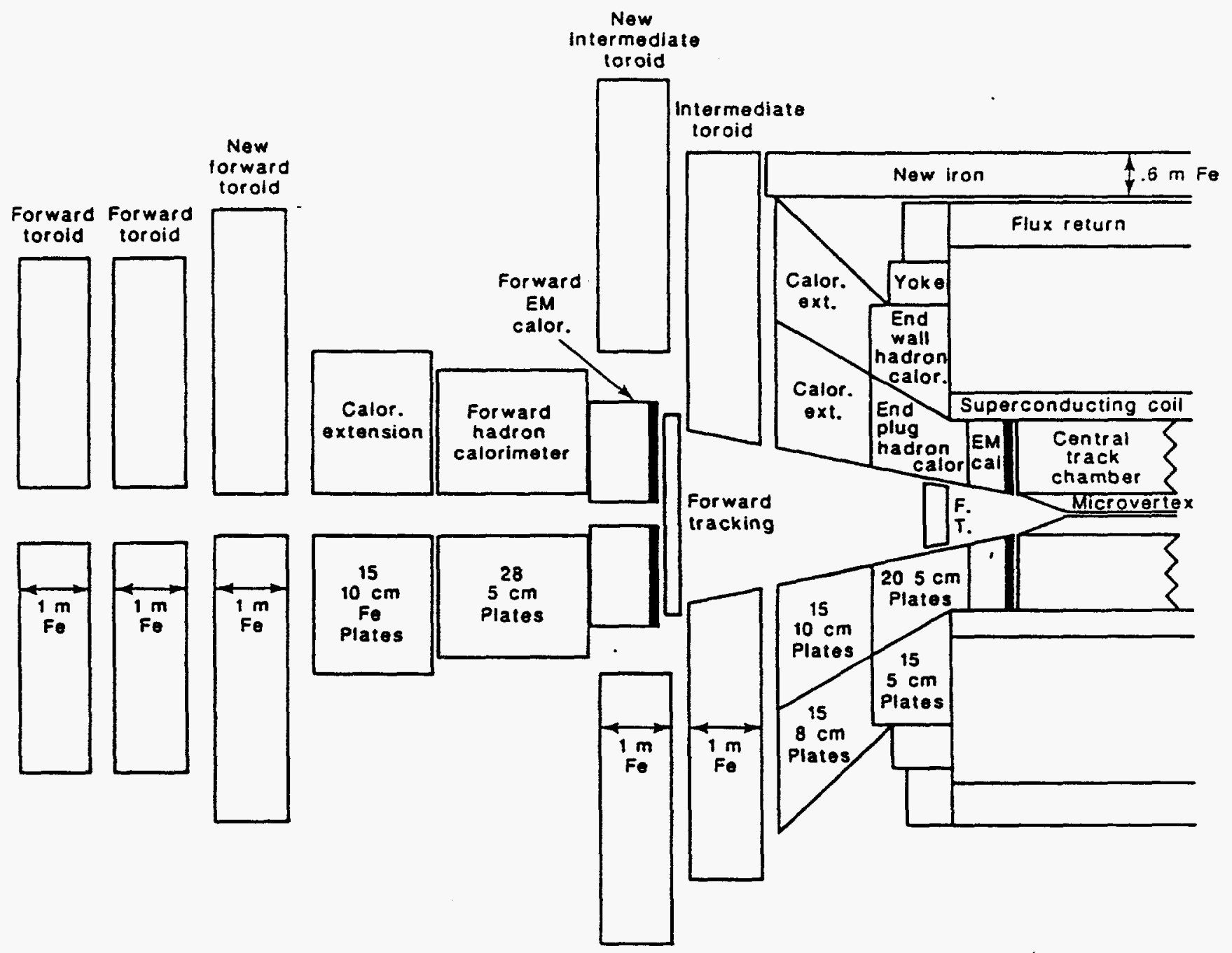

Fig. 7. Upgraded CDF Detector 
Table 6

Parameters of Upgraded COF Detector

1. Electromagnetic Calorimeters

\begin{tabular}{|c|c|c|c|c|}
\hline & $\begin{array}{c}\text { Central } \\
\text { (new) }\end{array}$ & $\begin{array}{l}\text { End Plug } \\
\text { (new) }\end{array}$ & $\begin{array}{l}\text { Forward } \\
\text { (existing) }\end{array}$ & $\begin{array}{l}\text { Forward } \\
\text { (new) }\end{array}$ \\
\hline$\left|y_{\min }\right|$ & 0.0 & 1.0 & 2.4 & 2.4 \\
\hline$\left|y_{\max }\right|$ & 1.1 & 2.4 & 4.5 & 4.5 \\
\hline$\theta_{\min }(\mathrm{rad})$ & 0.64 & 0.175 & 0.0221 & 0.0221 \\
\hline$\theta_{\max }(\mathrm{rad})$ & 1.57 & 0.68 & 0.175 & 0.175 \\
\hline$\Delta y(r>50 \mathrm{~cm})$ & 0.03 & 0.03 & 0.03 & 0.03 \\
\hline$\Delta \emptyset(r>50 \mathrm{~cm})$ & 0.03 & 0.03 & 0.03 & 0.03 \\
\hline Tower size $(\mathrm{cm})$ & $5 \times 5-5 \times 8$ & $5 \times 5-1.1 \times 1.1$ & $4 \times 4-1.5 \times 1.5$ & $4 \times 4-1.5 \times 1.5$ \\
\hline No. of Towers & $15.4 K$ & $19.5 K$ & $19.7 K$ & $19.7 K$ \\
\hline Longitudinal Segments & 2 & 2 & 1 & 1 \\
\hline Absorb. Type & uranium & uranium & lead & lead \\
\hline Absorb. Thick (cm) & 0.3 & 0.3 & 0.48 & 0.56 \\
\hline Sampling & $0.4 \mathrm{~cm} \mathrm{liq.}$ & $0.4 \mathrm{~cm} \mathrm{liq.}$ & gas & gas \\
\hline Gap Thick. $(\mathrm{cm})$ & 0.5 & 0.5 & 2.06 & 1 \\
\hline No. of Samples & 27 & 42 & 30 & 10 \\
\hline$R_{\min }(\mathrm{cm})$ & 170.0 & 33.6 & 16 & 17.5 \\
\hline$R_{\max }(\mathrm{cm})$ & 191.6 & 153.6 & 126 & 139.9 \\
\hline$z_{\min }(\mathrm{cm})$ & 0 & 190 & 715 & 791 \\
\hline$z_{\max }(\mathrm{cm})$ & 250 & 223.6 & 791 & 807 \\
\hline Length $(\mathrm{cm})$ & 250 & 33.6 & 76 & 15.6 \\
\hline Depth (abs. Ten.) & 0.8 & 1.3 & 0.8 & 0.3 \\
\hline Depth (rad. Ten.) & 25.3 & 39.4 & 26 & 10 \\
\hline Weight (tons) & 87 & 36 & 29 & 11 \\
\hline
\end{tabular}


(Table 6 cont'd.)

2. Hadronic Calorimeters

\begin{tabular}{|c|c|c|c|}
\hline $\begin{array}{l}\text { Central } \\
\text { Hadron } 1\end{array}$ & $\begin{array}{l}\text { Central } \\
\text { Hadron } 2\end{array}$ & $\begin{array}{l}\text { End wall } \\
\text { (existing) }\end{array}$ & $\begin{array}{l}\text { End wall } \\
\text { (new) }\end{array}$ \\
\hline 0 & 0 & 0.7 & 0.93 \\
\hline \multirow[t]{3}{*}{0.9} & 0.8 & 1.3 & 1.32 \\
\hline & & 0.52 & 0.52 \\
\hline & & 0.92 & 0.75 \\
\hline 0.06 & 0.06 & 0.06 & 0.06 \\
\hline 0.06 & 0.06 & 0.06 & 0.06 \\
\hline $13 \times 13$ & $15 \times 15$ & $9 \times 9$ & $13 \times 13$ \\
\hline $3.1 \mathrm{~K}$ & $2.8 K$ & $2.2 \mathrm{~K}$ & $1.4 K$ \\
\hline 1 & 1 & 1 & 1 \\
\hline uranium & iron & iron & iron \\
\hline 0.6 & 6 & 5 & 8 \\
\hline $0.4 \mathrm{~cm} \mathrm{liq.}$ & scint or gas & gas & gas \\
\hline 0.5 & 2 & 2 & 2 \\
\hline 50 & 20 & 15 & 15 \\
\hline 195 & 250 & 148 & 208.4 \\
\hline 250 & 410 & & 339.1 \\
\hline 0 & 0 & 259 & 364 \\
\hline 250 & 250 & 364 & 514 \\
\hline 250 & 250 & 105 & 150 \\
\hline 3 & 7.1 & 4.4 & 7.1 \\
\hline 664 & 995 & 290 & 690 \\
\hline
\end{tabular}


(Table 6 cont'd.)

2. Hadronic Calorimeters (cont'd.)

\begin{tabular}{|c|c|c|c|}
\hline $\begin{array}{l}\text { End Plug } \\
\text { (existing) }\end{array}$ & $\begin{array}{l}\text { Eng Plug } \\
\text { (new) }\end{array}$ & $\begin{array}{c}\text { Forward } \\
\text { (existing) }\end{array}$ & $\begin{array}{l}\text { Forward } \\
\text { (new) }\end{array}$ \\
\hline 1.3 & 1.3 & 2.4 & 2.4 \\
\hline 2.4 & 2.4 & 4.2 & 5.5 \\
\hline 0.175 & 0.175 & 0.0309 & 0.0082 \\
\hline 0.52 & 0.52 & 0.175 & 0.175 \\
\hline 0.06 & 0.06 & 0.06 & 0.06 \\
\hline 0.06 & 0.06 & 0.06 & 0.06 \\
\hline $10 \times 12-2.2 \times 2.2$ & $14 \times 14-3 \times 3$ & $8 \times 8-3 \times 3$ & $8 \times 8-3 \times 3$ \\
\hline $3.9 \mathrm{~K}$ & $3.9 \mathrm{~K}$ & $4.9 \mathrm{~K}$ & $5.3 K$ \\
\hline 1 & 1 & 1 & 1 \\
\hline iron & iron & iron & iron \\
\hline 5 & 10 & 5 & 10 \\
\hline gas & gas & gas & gas \\
\hline 2 & 2 & 3.18 & 2 \\
\hline 20 & 15 & 28 & 15 \\
\hline 40 & 65 & 25 & 8.5 \\
\hline 129 & 210 & 143 & 184 \\
\hline 226 & 366 & 809 & 1038 \\
\hline 366 & 546 & 1038 & 1218 \\
\hline 140 & 180 & 229 & 180 \\
\hline 5.9 & 8.8 & 8.2 & 8.8 \\
\hline 150 & 480 & 448 & 550 \\
\hline
\end{tabular}


3. Tracking Devices

\begin{tabular}{ll} 
Silicon Vertex Detector & \\
\hline Rapidity range & -3.0 to 3.0 (1st layer) \\
Length (cm) & -2.3 to 2.3 (3rd layer) \\
No. of Layers & 67 \\
Radii (cm) & 4 \\
Strip pitch & $2.94 .4 .87,6.79,8.72$ \\
Readout pitch & $25 \mu \mathrm{m}$ \\
No. of readout channels & $50 \mathrm{and} 100 \mu \mathrm{m}$ \\
Double track separation & $\approx 40 \mathrm{~K}$ \\
Resolution & $200-400 \mu \mathrm{m}$ \\
& $10-25 \mu \mathrm{m}$ \\
Wire Chamber Vertex Detector & \\
Rapidity range & $-2.4 \mathrm{to} 2.4$ (all layers) \\
Length & $120 \mathrm{to} 340 \mathrm{~cm}$ \\
No. of layers & 25 \\
Inner radius & $10 \mathrm{~cm}$ \\
Outer radius & $30 \mathrm{~cm}$ \\
No. of wires & $9 \mathrm{~K}$ \\
No. of strips or charge div. & $9 \mathrm{~K}$ \\
Max. drift distance & $1.5 \mathrm{~mm}$ \\
Double track separation & $350-500 \mu \mathrm{m}$ \\
Position resolution & $60-100 \mu \mathrm{m}$
\end{tabular}

\begin{tabular}{lll} 
Central Drift Chamber & Region I & Region II \\
\hline Rapidity range & -2.3 to 2.3 inner layer & -1.6 to 1.6 inner layer \\
& -1.6 to 1.6 outer layer & $-1.0 \mathrm{co} 1.0$ outer layer \\
Length & $340 \mathrm{~cm}$ & $340 \mathrm{~cm}$ \\
Inner radius & $35 \mathrm{~cm}$ & $70 \mathrm{~cm}$ \\
Outer radius & $70 \mathrm{~cm}$ & $140 \mathrm{~cm}$ \\
No. of layers & 35 & 40 \\
No. of sense wires & $19 \mathrm{~K}$ & $26 \mathrm{~K}$ \\
No. of strips or charge div. & $9 \mathrm{~K}$ & $13 \mathrm{~K}$ \\
Max drift distance & $3 \mathrm{~mm}$ & $5 \mathrm{~mm}$ \\
Double track separation & $1 \mathrm{~mm}$ & $2-3 \mathrm{~mm}$ \\
Position resolution & $<150 \mu \mathrm{m}$ & $<150 \mu \mathrm{m}$ \\
& & \\
Forward Tracking & $\frac{\text { Radial Chamber }}{\mathrm{Ealor}}$ & $\mathrm{Chamber}$ \\
Rapidity range & $2.4 \mathrm{to} 4.0$ & $350 \mathrm{~cm}$ \\
Length (wire) & $42 \mathrm{~cm}$ & $30 \mathrm{~cm}$ \\
Inner radius & $11 \mathrm{~cm}$ & 175 \\
Outer radius & $53 \mathrm{~cm}$ & 20 \\
No. of layers (both sides) & 20 & $6 \mathrm{~K}$ \\
No. of sense wires & $7 \mathrm{~K}$ & $3 \mathrm{~K}$ \\
No. of strips or charge div. & $3 \mathrm{~K}$ & $5 \mathrm{~mm}$ \\
Max. drift distance & $5 \mathrm{~mm}$ & $3 \mathrm{~mm}$ \\
Double track separation & $3 \mathrm{~mm}$ & $<200 \mu \mathrm{m}$ \\
Position resolution & $<200 \mu \mathrm{m}$ &
\end{tabular}


4. Muon System

Rapidity coverage

Chamber resolution

No. of track layers

central

intermediate

forward

Chamber area $\left(\mathrm{m}^{2}\right)$

central

intermediate

forward

Total

No. of wires

central

intermediate

forward

Total

Toroids (tons)

forward (exist)

forward

intermediate

Total

Additional steel (tons) central
-4.0 to 4.0

200-300 um

4

6

8

1936

1328

960

4224

$24 K$

$77 K$

$72 K$

$53 K$

1520

1120

3364

6004

2300

Electronics Channel count silicon vertex detector wire vertex detector central drift chamber forward radial chamber forward calor. chamber

$40 \mathrm{~K}$

$18 \mathrm{~K}$

$67 K$

10K

9K

EM calorimeter

Hadron Calorimeter

109K

Muon System

$28 \mathrm{~K}$

160K ( 2 pulse heights plus 1 time)

Total

$401 k$ (excl. silicon) 
Table 7

Summary of Upgraded CDF Detector Performance

Central Tracking

EM Calorimetry

Central

End Plug

Forward

Hadron Calorimetry

Central

End Wall

End Plug

Forward

Muon System

Central

Intermediate

Forward
$\Delta \mathrm{P}_{\mathrm{t}} \approx 5 \times 10^{-4} \mathrm{p}_{\mathrm{t}}{ }^{2}$

Shower position

$2-3 \mathrm{~mm}$

$1-3 \mathrm{~mm}$

$1-3 \mathrm{~mm}$

Energy Resolution

$15 \% / \sqrt{\mathrm{E}}$

$15 \% / \sqrt{\mathrm{E}}$

$25 \% / \sqrt{E}$

Energy Resolution

$50 \% / \sqrt{\mathrm{E}}$

$50-100 \% / \sqrt{E}$

$100 \% / \sqrt{E}$

$110 \% / \sqrt{E}$

Momentum Resolution

(1) $100 \mathrm{GeV}$

a large momentum

$5 \%$

$16-17 \%$

$16-17 \%$
$\Delta P_{t} \approx 5 \times 10^{-4} P_{t}^{2}$

$\Delta P_{t} \approx 2.5 \times 10^{-4} P_{t}^{2}$

$\Delta P_{t} \approx 2.5 \times 10^{-4} P_{t}^{2}$ 
each, fifteen $8 \mathrm{~cm}$ plates, fifteen $10 \mathrm{~cm}$ plates, and fifteen $10 \mathrm{~cm}$ plates, respectively. In the case of the first two, it might be possible to weld the additional plates to the existing units, though it would require some reworking of the units. For the forward calorimeter, and very likely for the others, one would add an additional back-up unit which is supported independently, but meshes well with the existing unit. A slight displacement or rotation of the new units would ensure that the small cracks that invariably exist due to chamber edges and readout cables would not line up with those of the existing units.

The electromagnetic calorimeters are 19 and 26 radiation lengths thick for the end plug and forward calorimeters respectively, and it would be desirable to add an additional 5 and 10 radiation lengths to the front of each of these. Because space is limited, and because the end plug EM calorimeter is rather small compared to the other modifications, we in fact assume that it is replaced entirely with a construction similar to that to be used for the central EM calorimeter.

In the case of the central calorimeters, which are currently only equivalent to $110 \mathrm{~cm}$ of iron, there exists little additional space. It therefore seems necessary to discard these completely and replace them with new units of similar size but much higher density. Uranium is probably the material of choice for at least the first four or five absorption lengths. The minimum space available, from the coil to the yoke, is $2.4 \mathrm{~m}$. We suggest twenty-seven $3 \mathrm{~mm}$ uranium plates for the electromagnetic compartment, fifty $6 \mathrm{~mm}$ uranium plates for the "precision" hadron calorimeter, and twenty $6 \mathrm{~cm}$ iron plates for the rest of the hadron calorimeter. This yields a total of 11 absorption lengths at $90^{\circ}$ in a space of $2.4 \mathrm{~m}$.

A "very forward" calorimeter must be utilized to cover the rapidity region 4.0-5.5. It is likely that this calorimeter will already be added in the course of CDF upgrades at the Tevatron. On one side, this region is covered by the forward spectrometer.

Segmentation. As far as segmentation of the calorimeter is concerned, finer is better. There is, however, little to be gained in tower sizes smaller than the electromagnetic or hadronic shower size. The two primary criteria for the electromagnetic calorimeter are that one be able to determine the position of an electron shower to an accuracy on the order of 1-2 mm and that one be able to identify electrons with reasonable efficiency. On the 
basis of Snowmass 1984 studies we choose a segmentation of $0.03 \times 0.03$ ( $\Delta y \times$ $\Delta$ ) for the electromagnetic calorimeter. A reasonable segmentation for the hadronic calorimeter would be one half that of the electromagnetic, namely $0.06 \times 0.06$. With two longitudinal segments in the electromagnetic calorimeter and two in the hadronic there are a total of $137 \mathrm{~K}$ channels.

Sampling. For the readout, gas calorimetry would be employed for the end plug and forward regions, and possibly also for the end walls. There is certainly some concern as to whether it can survive the high rates and radiation. Recent measurements indicating that chambers can survive total charge collection rates of 1 Coulomb per $\mathrm{mm}$ imply that if the chamber is built cleanly and the proper gas mixture is used, it should perform stably at luminosities up to $10^{32} \mathrm{~cm}^{-2} \mathrm{~s}^{-1}$. The particle rate incident on a given tower of size $0.06 \times 0.06$ would be $10^{5}$ per second and the current per wire can be kept below 0.1 uA.

For the central region (and possibly also the end walls), the readout is less certain at this time. Scintillator techniques could probably be used for segmentations as small as $0.06 \times 0.06$ (adequate for the hadron calorimeter) if fibers can be used for the light guides or if avalanche photodiodes improve substantially. For the segmentation desired for the electromagnetic calorimeter and the precision hadron calorimeter, readout with silicon, a warm sampling liquid, or gas chambers would be required. Neither of the first two options is yet proven, though it is planned to use warm liquid readout for the UA1 upgrade; it therefore seems reasonable to assume that this will be a realistic possibility by the 1990's, at least for relatively small calorimeters. Gas sampling should be fine for the coarse hadron calorimeter, and must be considered the fallback solution for the others.

\section{Track Chambers}

Central Track Chamber. The region available for the central track chamber should be sufficient if the wire spacing is decreased to keep the maximum drift time below $100 \mathrm{nsec}$, and if the two-track separation is improved.

Assuming that an interaction occurs on average every $100 \mathrm{nsec}$, we choose a maximum drift distance of $5 \mathrm{~mm}$ near the outer radii of the drift chamber and 3 $\mathrm{mm}$ near the inner radii. We somewhat arbitrarily assume 35 layers of wires with $3 \mathrm{~mm}$ drift distance between radii of 35 and $70 \mathrm{~cm}$ (region I) and 40 layers of wires with $5 \mathrm{~mm}$ drift distance between radii of 70 and $140 \mathrm{~cm}$ (region II). 
It may be desirable to isolate the gas flow in the two regions so that a slower gas could be used in region I if desired. With appropriate shaping, it has been possible to obtain pulse widths (near the base) of $20 \mathrm{nsec}$, and we therefore assume a two track resolution of $1 \mathrm{~mm}$ for the inner wires. Such a two track separation has been achieved in current microvertex detectors, and is approximately 2-4 times better than what has so far been achieved in large detectors. This yields 2000 position bins in the first chamber layer. Since the innermost layer of the chamber covers about 2.3 units rapidity, the average track multiplicity from minimum bias events will be 27 , an occupancy rate of slightly more than $1 \%$ which is approximately one third of the average occupancy rate in the inner layer of COF operating at the Tevatron. For jets of very large transverse momentum, it will of course be impossible to reconstruct tracks near the core; but this segmentation should be adequate for the identification of electrons and muons produced in the decays of $W$ or $Z$ bosons, in the decays of $b$ quarks up to transverse momenta of several tens of $\mathrm{GeV}$, and in the decays of the top or heavier quarks up to transverse momenta of several TeV. With the above configuration, the central track chamber would include approximately $45 \mathrm{~K}$ wires. We assume that an additional $22 \mathrm{~K}$ channels are devoted to either charge division or to strips or a mixture of the two.

Microvertex Chamber. The region inside the Central Track Chamber would be filled by a wire chamber microvertex detector extending from a minimum radius of $10 \mathrm{~cm}$ to $30 \mathrm{~cm}$. There are many different techniques currently being used to build such chambers and we do not pretend to pick the best one here. We assume that the maximum drift distance will be $1.5 \mathrm{~mm}$ and that there are approximately 25 layers thus yielding a total of $9 \mathrm{~K}$ sense wires. Usage of a gas with drift velocity on the order of $60 \mathrm{nsec} / \mathrm{mm}$ should enable a position resolution of order 60-80 $\mu \mathrm{m}$, a two-track separation of 350-500 $\mu \mathrm{m}$, and a maximum drift time of $90 \mathrm{nsec}$. We allow for an additional $9 \mathrm{~K}$ electronics channels for charge division or strip readout.

silicon Vertex Detector. Inside the microvertex chamber will be a silicon vertex detector, which we take to be the one to be built for CDF at the Tevatron. It will include four layers, each in the shape of a dodecagon, at radii of $2.94 \mathrm{~cm}, 4.87 \mathrm{~cm}, 6.79 \mathrm{~cm}$, and $8.72 \mathrm{~cm}$. Each layer is planned to be $67 \mathrm{~cm}$ long thus yielding rapidity coverages of \pm 3.0 units for the innermost layer and \pm 2.3 units for the third layer. The strip spacing will be $25 \mu \mathrm{m}$ 
while the readout pitch will vary from $50 \mu \mathrm{m}$ for the inner layer to $100 \mu \mathrm{m}$ for the outer layer. Readouts on both ends of the strips require a total of approximately 40K channels. The electronics must, of course, be in integrated circuit form with a high degree of multiplexing.

Forward Tracking chambers. We assume that the forward tracking will be similar to that planned for CDF at the Tevatron, i.e., a radial wire chamber mounted in, or just at the end of, the end plug, plus chambers just in front of the forward electromagnetic calorimeter. For the former we assume a minimum radius of $11 \mathrm{~cm}$, a maximum radius of $53 \mathrm{~cm}$ and a distance from the intersection point of $300 \mathrm{~cm}$. This covers the rapidity interval of 2.4 to 4.0. The maximum drift distance would vary from $5 \mathrm{~mm}$ at the outer radius to $1 \mathrm{~mm}$ at the inner radius. The counting rate nearest the beam pipe would be $2.5 \times 10^{4} / \mathrm{sec} / \mathrm{cm}$ of wire length. Twenty planes of 350 sense wires/plane yield a total of $7 K$ wires for the two sides.

For the chamber just in front of the calorimeter, one can imagine a relatively conventional construction of planar drift chambers, with either charge division or cathode strips to provide space points. Assuming a maximum drift distance of $5 \mathrm{~mm}$, one would have 290 wires/plane. Again assuming twenty layers, one estimates a total of $6 \mathrm{~K}$ wires. The maximum counting rate/cm of wire length, which occurs at a rapidity of 4.0 , would be $1.5 \times 10^{4} / \mathrm{sec}$.

\section{Muon Detection}

If the presently planned muon upgrades for COF are implemented, the total steel-equivalent thickness of absorber, including the calorimetry improvements discussed above, would range from $240 \mathrm{~cm}$ at $90^{\circ}$ to $490 \mathrm{~cm}$ in the forward direction. The same side walls and toroids could be utilized, though additional ones would need to be added if it were desired to extend the sign determination in the forward direction to momenta above $500 \mathrm{GeV}$. We propose adding an additional forward toroid and an additional toroid at intermediate angles, each of $1 \mathrm{~m}$ thickness. We also propose adding an additional $60 \mathrm{~cm}$ of iron on the sides and top of the yoke. Then the minimum total thickness in the central region would be $3 \mathrm{~m}$ of steel equivalent, and in the forward region there would exist $5.9 \mathrm{~m}$ of absorber with $3 \mathrm{~m}$ magnetized steel for the momentum measurement. The toroids would then cover the region $2^{\circ}$ to $38^{\circ}$ and would provide a momentum resolution of order 17-20\% for momenta up to $900 \mathrm{GeV}$ and sign determination to nearly $2 \mathrm{TeV}$. The existing chambers could probably be 
utilized if it were only desired to identify muons offline. New chambers, or at least additional ones, would be required to trigger on muons. We assume two planes of chambers before and after each toroid, and a maximum drift distance of $1 \mathrm{~cm}$. For muons away from the core of jets in the central rapidity region, the central track chamber should be able to provide a momentum resolution of $5 \%$ at $100 \mathrm{GeV}$ and sign determination to nearly $1 \mathrm{TeV}$.

\section{Triggering}

We do not discuss the triggering in any detail but refer one to the discussions in the 1984 Snowmass Summer Study, and the recent Triggering Workshop at Fermilab. A few brief comments concerning the philosophy relative to muon triggers are perhaps useful, however. The total rate of muons outside $3 \mathrm{~m}$ of steel is estimated to be $10^{4} / \mathrm{sec}$ for a luminosity of $10^{32} \mathrm{~cm}^{2} \mathrm{~s}^{-1}$. A simple level 1 trigger, using the angle of the track segment outside the steel in the central region and the cells hit in the forward and intermediate toroids, can select only those muons consistent with a transverse momentum greater than 5-6 GeV. This reduces the trigger rate to less than $1 \mathrm{kHz}$. A more sophisticated level 2 trigger, which uses the drift time information in the central track chamber and the forward muon chambers, should be able to make a transverse momentum cut of order $15-20 \mathrm{GeV}$. This cut, taken together with information in jets, missing transverse momentum, and other leptons, should be sufficient to allow efficient triggering on most topics of interest. The trigger rate for single, isolated muons, with no other distinguishing characteristic in the event, might have to be prescaled.

\section{Electronics}

We do not attempt to discuss the electronics in any detail other than to note a few of the general features. To achieve charge collection times of a few hundred nanoseconds, it will almost certainly be necessary to distribute the preamplifiers throughout the calorimetry; the actual number of preamplifiers may in fact be larger than the number of channels indicated to reduce the source capacitance. It is assumed that the cost of the preamplifiers will be relatively small since it is very likely that they will be fully integrated. While the average time between interactions will be of order 100 nsec, the actual time will vary statistically (though always quantized to the bunch crossing times). Since the charge collection time and the level 1 trigger time will be longer than the time between interactions, it will be neces- 
sary to pipeline the information over a period of several hundred nanoseconds to a microsecond. It is assumed that either flash ADC's with local data compaction or an analog sampler similar to that being developed for SLD will be used. Given the number of channels required, nearly all of the electronics will be integrated circuits.

The basic data acquisition system and trigger of CDF would still be utilized, though individual modules (such as scanners, track processors, level 2 trigger modules, and level 3 processors) would be replaced.

\subsubsection{Upgraded DO Detector}

The DO detector is a non-magnetic general detector whose primary foci are the detection of electrons, muons, jets and missing transverse energy. Particular attention has been paid to the design of good uranium-copper/ liquid argon calorimetry, without projective cracks.

The main areas of deficiency with respect to SSC usage are the following: (1) insufficient granularity of tracking cells for the high rate environment, (2) calorimeter depth too small for SSC energies, (3) too little iron toroid depth to give clear muon signatures and muon sign determination in the multiTeV range and (4) front-end electronics and trigger system too slow by SSC standards. Figure 8 shows a schematic plan or elevation view of of after upgrade. The improvements proposed are listed and discussed below. The corresponding parameters are listed in Table 8 . The performance characteristics are summarized in Table 9 .

\section{Calorimetry}

To improve the hadronic calorimetry we propose adding a coarse Fe-sampling calorimeter section behind the present of liquid argon devices. This steel would be the first section of the muon momentum system (i.e., magnetized). Since the transition from liquid argon to Fe occurs well past shower maximum $\left(t_{\max } \simeq 3.8 \lambda_{0}\right.$ at $\left.1000 \mathrm{GeV}\right)$, sampling can be relatively coarse in the added section. Interspersing 15 layers of chambers in the Fe-catcher section would give about $6 \mathrm{~cm}$ sampling $\left(1 / 3 \lambda_{0}\right)$. The space occupied and iron weight involved would be almost identical to the present o muon toroid system. One would probably make new iron stacks from rolled steel plate and set aside the current $0 \phi$ toroid steel for use in the new muon toroids. The existing $0 \phi$ coils should be usable for the upgraded catcher calorimeters. 
Upgraded DO
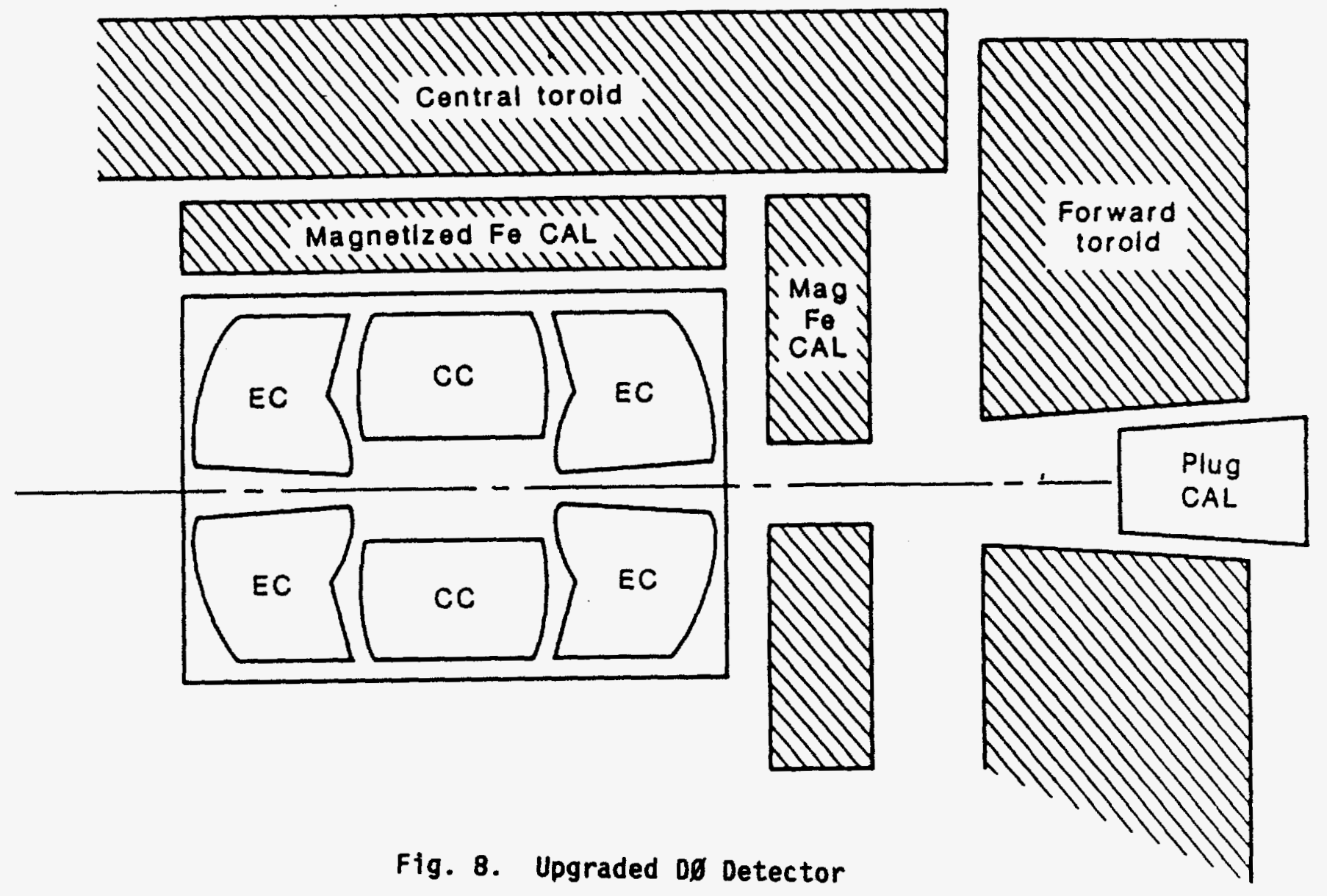
Inner Vertex Chamber (upgrade)

$\begin{array}{ll}r \text { inner } & 5 \mathrm{~cm} \\ r \text { outer } & 17 \mathrm{~cm} \\ \text { length } & 100 \mathrm{~cm} \\ \text { No. of layers } & 20 \\ \text { No. of wires } & 7000 \\ \text { sense wire spacing } & 2 \mathrm{~mm} \\ \text { resolution } & <100 \mathrm{~mm} \\ \text { No. of signals (with chg. div.) } & 14,000\end{array}$

\section{Transition Radiation Detector (upgrade)}

$\begin{array}{ll}r \text { inner } & 17 \mathrm{~cm} \\ r \text { outer } & 50 \mathrm{~cm} \\ \text { length } & 200 \mathrm{~cm} \\ \text { No. of X-ray detectors } & 3 \\ \text { No. of wires } & 1470 \\ \text { wire spacing } & 5 \mathrm{~mm} \\ \text { No. of cathode pads } & 15,000 \\ \text { pad area } & 10 \mathrm{~cm}^{2}\end{array}$

\section{Central Drift Chamber (upgrade)}

$\begin{array}{ll}r \text { inner } & 50 \mathrm{~cm} \\ r \text { outer } & 75 \mathrm{~cm} \\ \text { length } & 270 \mathrm{~cm} \\ \text { No. of layers } & 20 \\ \text { No. of wires } & 20,000 \\ \text { sense wire spacing } & 4 \mathrm{~mm} \\ \text { resolution } & <200 \mu \mathrm{m} \\ \text { No. of signals (with chg. div.) } & 40,000\end{array}$

Forward Orift Chambers (upgrade)

(both ends)

$r$ inner

$7 \mathrm{~cm}$

$r$ outer

$40 \mathrm{~cm}$

21

22

No. of layers (each end)

No. of radial sense wires

$115 \mathrm{~cm}$

$140 \mathrm{~cm}$

28 [14 radial, 14 azimuth]

No. of azimuthal sense wires

inner radial sense wire spacing

6160

17920 [along octagon]

azimuthal sense wire spacing

resolution

No. of signals (with chg. div.

on azimuth. wires)

$2 \mathrm{~mm}$

$4 \mathrm{~mm}$

$<200 \mu \mathrm{m}$

42,000 
Central Liquid Argon Calorimeter (existing)

(Table 8 cont'd.)

$r$ inner
$r$ outer
length
gap
absorber

depth

depth segments

transverse segmentation

tower size

No. of channels

weight
$75 \mathrm{~cm}$

$260 \mathrm{~cm}$

$261 \mathrm{~cm}$

$2 \times 2.3 \mathrm{~mm} \mathrm{Ar}+1 \mathrm{~mm} \mathrm{G10}$

$3 \mathrm{~mm} U(E M)(0.94 \mathrm{rad}$. len.)

$6 \mathrm{~mm} U$ (fine Had) $(0.06 \lambda)$

$43 \mathrm{~mm}$ Cu (coarse Had) $(0.3 \lambda)$

20 rad. lengths (EM)

7.5 abs. lengths (Total)

4 (EM)

3 (fine Had)

1 (coarse Had)

$0.1 \times 0.1(y, \infty)$

$0.05 \times 0.05$ (third EM section)

$8 \times 8 \mathrm{~cm}^{2}$

$16 \mathrm{~K}$

350 metric tons

Endcap Liquid Argon Calorimeter (existing) (both ends)

$r$ inner

$r$ outer

z1

$\mathrm{z2}$

gap

absorber

depth

depth segments

transverse segmentation

tower size (minimum)

No. of channels

weight

\section{Central Catcher Calorimeter (upgrade)}

$r$ inner

$r$ outer

length

No. of samples

sampling

segmentation

depth

No. of channels

weight

B-field
$300 \mathrm{~cm}$

$410 \mathrm{~cm}$

$730 \mathrm{~cm}$

15

gas

$0.2 \times 0.2(y, 0)$

5 abs. lengths

330

1300 metric tons

$1.8 \mathrm{~T}$ 
Endcap Catcher Calorimeter (upgrade)

(both ends)

21

$z 2$

$r$ inner

$r$ outer

No. of samples

sampling

segmentation

depth

No. of channels

weight

B-field
$435 \mathrm{~cm}$

$590 \mathrm{~cm}$

$40 \mathrm{~cm}$

$400 \mathrm{~cm}$

20

gas

$0.2 \times 0.2(y, \infty)$

5 abs. lengths

680

110 metric tons

1.8 T

Central Muon Toroid (partial upgrade)

$r$ inner
$r$ outer
length
weight
B-field
internal gaps
thickness

End Toroids (partial upgrade)

21

22

$r$ inner

$r$ outer

weight

B-field

internal gaps

thickness
$420 \mathrm{~cm}$

$620 \mathrm{~cm}$

$1200 \mathrm{~cm}$

7860 metric tons

$1.8 \mathrm{~T}$

1

12 abs. lengths
$600 \mathrm{~cm}$

$900 \mathrm{~cm}$

$42 \mathrm{~cm}$

$620 \mathrm{~cm}$

7000 metric tons

$1.8 \mathrm{~T}$

2

18 abs. lengths

Muon chambers (partial upgrade)

No. of layers (central)

No. of layers (ends)

total area

No. of wires (central and ends)

No. of cathode pads (2 per wire)

Total No. of readouts (2 time signals/wire

plus cathode pads)

9

12 each end $12000 \mathrm{~m}^{2}$

$25 K$

$50 K$

$100 \mathrm{~K}$ 
(Table 8 cont'd.)

SUMMARY CHANNEL COUNT

Vertex Chamber 14K

Transition Radiation Detectors 15K

Central Drift Chamber 40K

Forward Drift Chambers 42K

Central LA Calor. 16K

Endcap LA Calor. 24K

Catcher Calorimeters IK

Muon Chambers 100K

TOTAL

$\frac{100 K}{252 K}$ 
Table 9

Summary of Upgraded DØ Detector Performance

Tracking:

$\begin{array}{lr}\text { Inner Vertex Chamber resolution } & 60 \mu \mathrm{m} \\ \text { Central Drift Chamber resolution } & 175 \mu \mathrm{m} \\ \text { Forward Drift Chamber resolution } & 175 \mu \mathrm{m}\end{array}$

TRD:

e/r discrimination

$\geq 50: 1$

Calorimetry: $\quad \Delta E / E(E M)$

$0.15 / \sqrt{E}(\mathrm{GeV})$

$\triangle E / E$ (Hadron)

$0.5 / \sqrt{E}(\mathrm{GeV})$

e/ $/$ discrimination

$>1000: 1$

position resolution

$8 \mathrm{~mm} / \sqrt{E}(\mathrm{GeV})$

Muon System: $\quad \Delta p / p$ in multiple scattering limit

$15 \%$

$p_{t}$ limit for sign determination

$1.5 \mathrm{TeV} / \mathrm{c}$ 
The performance of the upgraded calorimetry should be excellent. With the catcher, we may expect EM energy resolutions of about $0.15 / \sqrt{E}(\mathrm{GeV})$ and hadron energy resolutions of about $0.45 / \sqrt{E}$; electron/hadron discrimination should be very good $\left(>10^{3}\right)$. Radiation hardness and ease of calibration are assured.

\section{Muon System}

A new muon toroid system should be built, together with the associated chambers for measurement of muon trajectories outside the steel. The first meter of magnetized steel resides in the catcher section of calorimetry. The added steel thickness is set primarily by the requirement that we have $\Delta p / p<$ $20 \%$ out to $p_{t}=1 \mathrm{TeV} / \mathrm{c}$. At $90^{\circ}$, this implies the addition of $2 \mathrm{~m}$ additional steel. For detection of $p_{t}=1 \mathrm{TeV} / \mathrm{c}$ muons at $20^{\circ}$, one needs to add to the $1.4 \mathrm{~m}$ of catcher steel a total of about $3 \mathrm{~m}$. We propose the addition of central and (two) end iron toroids with a geometry simply expanded from the present $D \emptyset$ design. The muon iron should be separated into $1 \mathrm{~m}$ thick shells to give 1 (central) or 2 (end) intermediate fixes on muon trajectories. The muon sampling layers outside the steel are split into two "decks" with a $1 \mathrm{~m}$ separation to give exit angle information. Each deck consists of three proportional drift tube layers with drift-time measurement of the bend coordinate. To obtain information on the non-bend coordinate, drift time is measured on both ends of each wire for a coarse measurement, and vernier cathode pad readout with two outputs per wire provides the more precise result. Each gap in the toroids is instrumented by similar 3-layer decks. The existing $D \emptyset$ toroid steel would be usable as a part of the new muon toroids. Newly required tube layers would consist of 2 decks at the exits and one interleaved deck for each meter of additional thickness. Tube layers at the entries to the magnetized iron catcher toroids are assumed to be taken from the present $D \emptyset$.

\section{Liquid Argon Electronics}

The liquid argon calorimeter electronics need to be upgraded. The sampling time for the $0 \emptyset$ electronics is about $3 \mu \mathrm{s}$. At the SSC $\left(\mathscr{L}=10^{32}\right.$ $\mathrm{cm}^{-2} \mathrm{sec}^{-1}$, this would mean that the calorimeter mixes the signals of some 30 interactions. Although the average energy per tower from extraneous events is only $300 \mathrm{MeV}$, this energy is in fact clustered and leads to significantly degraded calorimeter performance. 
Two changes thus need to be made. First, the sampling time should be reduced to optimize the balance between electronics noise and the overlap event background. Second, tagging of the time of energy deposit within each tower should be incorporated. A simple-minded timing scheme would record the last time of receiving charge from the calorimeter over a preset threshold. This scheme leads to considerable ambiguity since only one such time is kept. A preferable scheme would be the recording of a several microsecond history of charge receipt in each channel using low-precision flash ADC's. The increased data load imposed by such a scheme is at most 50K bytes, and considerable compaction could be made if this information is kept only for towers showing large energy deposits.

\section{Tracking}

The $D \emptyset$ tracking chambers have too long drift times and should be replaced by close-spaced wire chambers. We follow the Snowmass Dl tracking chamber in specifying 20 layers of sense wires (2 $\mathrm{mm}$ spacing) for the inner vertex chamber and 20 (28) layers (4 mm spacing) in outer (forward) chambers. Charge division is used for orthogonal coordinate readout.

The TRD system probably needs new chambers as we11; wire spacings need to be reduced to the minimum to control multinits and fine cathode pad readouts should be incorporated to give space-point measurement.

The tracking chamber system will be required to help reduce backgrounds to electron signatures in the non-magnetic environment. Particularly important are the tagging of conversion $e^{+} e^{-}$pairs through $d E / d x$ measurement in repeated layers. The chamber operation and electronics must have the capability of charge measurement and should be optimized for good two-track separation. The single track resolution is not at a high premium since momenta are not measured.

\section{Trigger System}

The $D$ trigger system needs some upgrading--primarily through the inclusion of a fast pretrigger system operating in the few hundred nanosecond range. In this time interval, simple sums of total $E_{T}$ or vector $P_{T}$ in channels whose charge exceeds a preset threshold can be formed. Quick decisions to proceed with triggering based on this information can be made which 
should reduce the rate by a factor of 100 . This trigger system which needs to be physically close to the detector to minimize delays, will necessarily operate at rather poor resolution owing to the large electronic noise contributions inherent in short time interval sampling. The output rate for this trigger level seems appropriate for the existing of trigger system, and no further modification should be necessary.

We have assumed that the upgraded $D \emptyset$ is to be combined with a new forward spectrometer covering the angular range $1<\theta<60 \mathrm{mr}$. The $0 \emptyset$ calorimetry gives hermetic coverage at larger angles. The small angle region opposite to the forward spectrometer will need to be instrumented, and we assume here that one of the existing $O \varnothing$ plug calorimeters will satisfy the need. It may need to be thickened, or another small angle calorimeter, far less ambitious than the forward spectrometer, but taking advantage of the long IR may have to be built.

\subsubsection{Upgraded UAT Detector}

The UAI detector is characterized by a transverse magnetic field of 0.7 Tesla, which allows momentum measurements down to very small angles and thus provides a very good match to a forward spectrometer which would cover rapidities larger than 3.0. The good performance in the e-coverage of a transversefield detector comes at the price of an azimuthal coverage which is much less uniform than that of a solenoidal field.

In looking into the upgrade problem, we have assumed that the calorimeter presently under construction by the UAI Collaboration will be used at the SSC. This calorimeter is based on uranium absorber with TMP warm liquid as active sampling medium. The TMP is contained in stainless steel boxes. Each readout pad has an area of $\sim 13 \times 12 \mathrm{~cm}^{2}$ in the barrel and $\sim 9.5 \times 9.2 \mathrm{~cm}^{2}$ in the end cap, so that at $90^{\circ}$ in the barrel at a distance of $\sim 130 \mathrm{~cm}$ from the interaction point, $\Delta \eta=\Delta \simeq 0.1$. The barrel modules and the forward modules have sixfold longitudinal segmentation.

The electromagnetic part of the calorimeter contains 40 depleted uranium plates $2 \mathrm{~mm}$ thick and is subdivided into 4 longitudinal readouts $\left(25 x_{0}\right)$. The hadronic part has $315 \mathrm{~mm} U$-plates, so that the total number of interaction lengths is $\sim 2(\lambda=120 \mathrm{~mm})$. 
To use UAT at much larger energies and at luminosities of $\leq 10^{32} \mathrm{~cm}^{-2} \mathrm{~s}^{-1}$ one must change the central tracking detector, which at present occupies a cylindrical volume $580 \mathrm{~cm}$ long and $130 \mathrm{~cm}$ in radius. Moreover, the depth of the calorimeter must be increased and the muon absorber improved by adding toroids to the existing ones. Finally the present scintillators, which are the active elements of the hadron catcher in the return yoke, must be replaced by gas tubes. We discuss these points in turn, and present the upgrade parameters in Table 10.

\section{The Central Detector}

With a minimum bunch spacing of $5 \mathrm{~m}$ and a luminosity of $10^{32} \mathrm{~cm}^{-2} \mathrm{~s}^{-1}$

one expects 0.2 events/bunch. Clearly the maximum drift distance for those wires which form an angle of about $100 \mathrm{mrad}$ with the forward direction must be very sma11. We propose a track detector which satisfies the requirement of a maximum drift distance of $\sim 1 \mathrm{~mm}$ based on the multidrift tube detector recently tested by Bouclier et al. (Appendix 2). Most of the tubes are placed parallel to the magnetic field, i.e., horizontally. A possible layout is shown in Fig. 9; it is formed by $30 \mathrm{~mm}$ wide hexagonal tubes (see Fig. 1 of Appendix 2) combined in $x$-layers, with tubes along the $x$-axis above and below the pipe, and a 2 -layer with tubes parallel to the beam at the periphery of the tracking detector volume. The $z$-layer is used to measure accurately the coordinates of the outgoing particles. All tubes have a 5-6 bit TDC for the drift measurement and $A D C$ 's at both ends for measuring the coordinate along the wire with an accuracy of $\sim 3 \times 10^{-3}$ of the tube length. In the Z-layer, there are two tubes for each value, meeting at the center as shown in Fig. $9 c$.

The lengths of the $X$-tubes vary with the $Z$ and $Y$ coordinates so that they are contained in a cylinder $\pm 260 \mathrm{~cm}$ long with a radius of $120 \mathrm{~cm}$. A cross section at $Z=0$ is shown in Fig. $9 b$.

Since the occupancy changes greatly with the angle $\theta$, the number of wires per tube can become smaller with increasing $\theta$. The dashed lines in Fig. 9a give a possible separation in angular regions: we assume 128 wires/tube (64 and 32 ) in the regions $A+B,\langle C$ and $D)$ respectively.

In each layer the tubes touch along one side so that their centers are at a distance of $30 / 1.15 \cong 26 \mathrm{~mm}$. A minimum ionizing particle crossing a layer of tubes with 128 wires gives on average 12 hits with a resolution per hit of 
Table 10

Added Components for Upgraded UAT Detector

\section{Central Detector}

Rapidity range

No. of layers

Total length of sensitive volume

Radius of sensitive volume

No. of tubes with 128 wires

No. of tubes with 64 wires

No. of tubes with 32 wires

Total number of drift wires (5-6 bit TDC)

Total number of $A D C$ for charge division

Barrel Uranium Calorimeter

Rapidity range

No. of added layers of $2 \mathrm{~mm} \mathrm{U}$

Readout granularity transversely

No. of added readouts longitudinally

Total number of added readout channels $\left(5 \times 5 \mathrm{~cm}^{2}\right)$

Total number of added readout channels $\left(10 \times 10 \mathrm{~cm}^{2}\right)$

\section{Endcap Uranium Calorimeter}

Rapidity range

No. of added layers of $2 \mathrm{~mm} U$

Readout granularity transversely $R \leq 100 \mathrm{~cm}$

Readout granularity transversely $(100 \leq R \leq 150 \mathrm{~cm})$

No. of added readout longitudinally

Total no. of added readout channels $\left(2.5 \times 2.5 \mathrm{~cm}^{2}\right)$

Total no. of added readout channels $\left(5 \times 5 \mathrm{~cm}^{2}\right)$

\section{Iron Calorimeter}

Iron thickness (existing)

Total Area of detectors in the Central Part (CP) No. of $30 \times 30 \mathrm{~cm}^{2}$ towers (CP)

Total Area of detectors in the end caps (EC)

No. of $15 \times 15 \mathrm{~cm}^{2}$ towers (EC)

\author{
-3.0 to 3.0 \\ 5 to 7 \\ $2 \times 260 \mathrm{~cm}$ \\ $120 \mathrm{~cm}$ \\ 554 \\ 554 \\ 988 \\ 140,544 \\ $2 \times 140,544$
}

-1.5 to 1.5

20

$5 \times 5 \mathrm{~cm}^{2}$ and $10 \times 10 \mathrm{~cm}^{2}$

2

$2 \times 7400$

$2 \times 2250$

3 to 1.5

20

$2.5 \times 2.5 \mathrm{~cm}^{2}$

$5 \times 5 \mathrm{~cm}^{2}$

2

$2 \times 9200$

$2 \times 3200$

$16 \times 5 \mathrm{~cm}=80 \mathrm{~cm}$ central

$25 \times 5 \mathrm{~cm}=125 \mathrm{~cm}$ end cap

$\sim 2000 \mathrm{~m}^{2}$

$2 \times 1100$

$\sim 7000 \mathrm{~m}^{2}$

$2 \times 1700$ 
Central Muon Toroids

Top toroid: $y_{1} / y_{2}$

Length of top toroid: $\Delta z$

Side toroids: $x_{1} / x_{2}$

Length of side toroid: $\Delta z$

Bottom toroid: $y_{1} / y_{2}$

Length of bottom toroid: $\Delta z$

Total Weight

Field

Internal gap

Decks of chambers ( 3 layers/each)

$420 / 620 \mathrm{~cm}$

$1100 \mathrm{~cm}$

$420 / 620$

$1100 \mathrm{~cm}$

$350 / 620 \mathrm{~cm}$

1100

7470 tons

$1.8 \mathrm{~T}$

1

3

End Toroids

Longitudinal dimensions: $z_{1} / z_{2}$

Angle of the hole

Transverse dimensions: $\Delta x$ and $\Delta y$

Total weight

Field

Internal gaps

Decks of chambers ( 3 layers each deck)

$570 / 820 \mathrm{~cm}$

$100 \mathrm{mrad}$

1100

$2 \times 2400$ tons

$1.8 \mathrm{~T}$

2

4

Muon Chambers

No. of layers (central)

No. of layers (end)

No. of wires (central and ends)

No. of cathode pads (2/wire)

Total No. of readouts (2 time signals/wire plus pads)

12 each end $25 \mathrm{~K}$

$50 \mathrm{~K}$

$100 \mathrm{~K}$ 


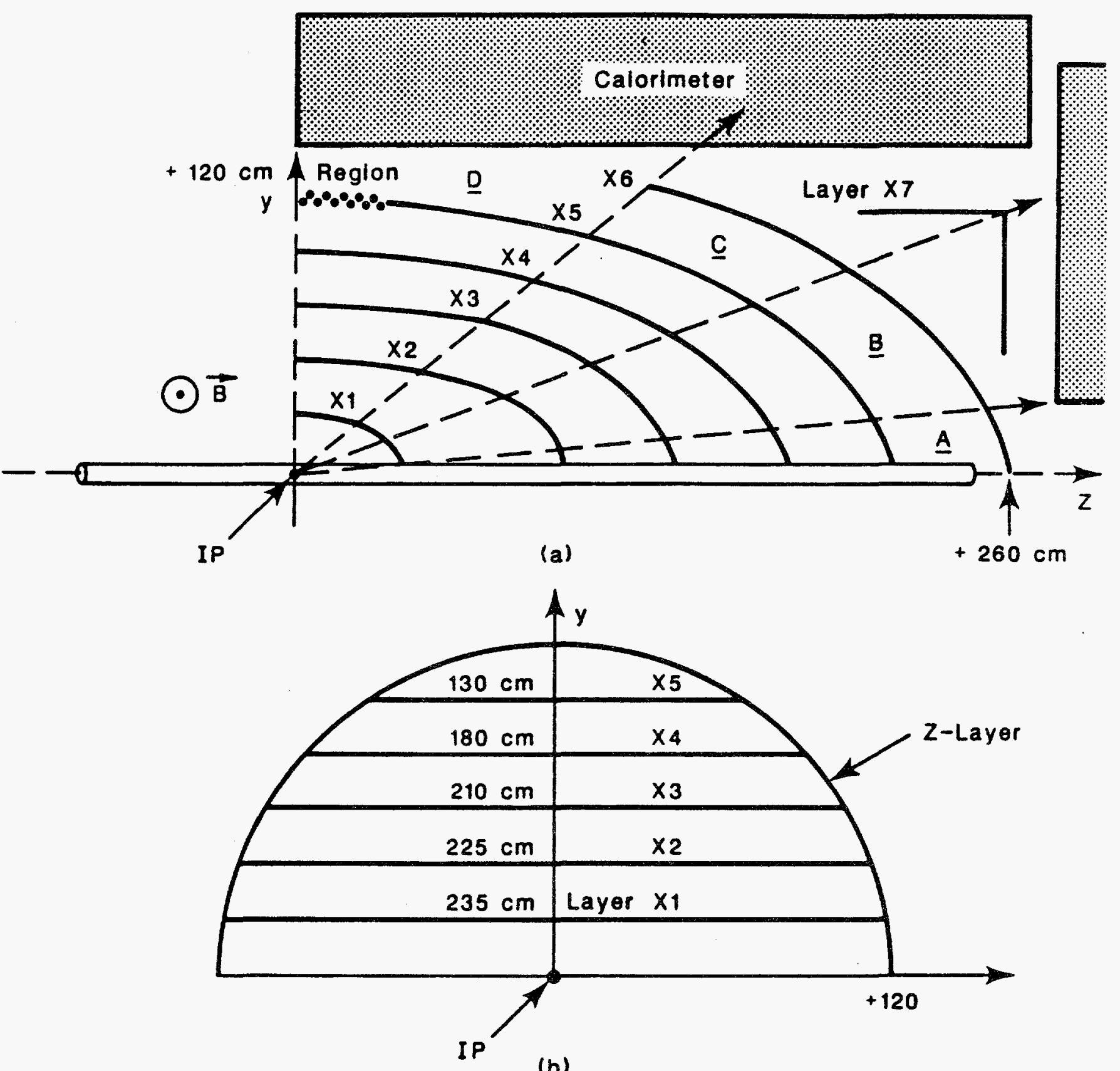

(b)

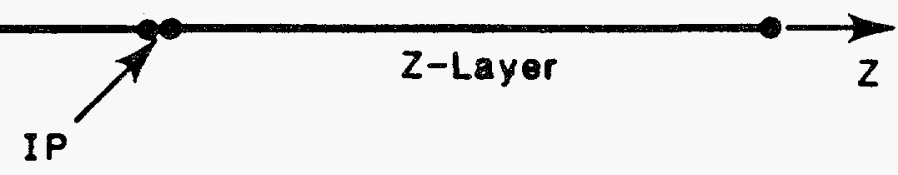

(c)

Fig. 9. Upgraded UA1 Detector Tracking System

(a) Vertical cross section parallel to the beam showing the various tube layers

(b) Vertical cross section parallel to the field

(c) Sketch of two tubes in the 2-layer 
about $100 \mu \mathrm{m}$. The two track resolution, for the same tube, is $\sim 300 \mu \mathrm{m}$ (Appendix 2). For the tubes with 64 (32) wires the numbers of hits per layer will be $9(6)$. The estimated precision $K=\Delta P_{T} / P_{T}^{2}$ in the various angular regions is given in Table 11.

The momentum resolution is reasonably good in the forward direction and becomes 10 times worse at $90^{\circ}$. The transverse field geometry, of course, implies that acceptance and resolution for particles forming a small angle with the magnetic field is greatly reduced.

The maximum thickness of the tube system of Fig. 9 is of the order of 0.03 radiation lengths, giving negligible scattering at these energies.

At present, for the production of few tubes the needed mechanical pieces are $300 \mathrm{SF}$ per tube, and the placement of wires in a 128 wire tube requires 10 days of technician time. Automatized methods are being developed for 2000 tubes. The electronics for each end has to be integrated and probabiy multiplexed to reduce costs for the readout. One needs a 5-6 bit TOC and $2 \times 8-9$ bit ADC's per wire.

\section{Uranium Calorimeter}

We propose to improve the granularity of the electromagnetic calorimeter, which at present is about $10 \times 10 \mathrm{~cm}^{2}$. For this reason the central detector will extend only to a radius of $\sim 120 \mathrm{~cm}$, leaving the possibility of inserting $10 \mathrm{~cm}$ of calorimeter with better spatial resolution. The present design foresees an uranium total thickness $40 \times 2 \mathrm{~mm}+31 \times 5 \mathrm{~mm}=235 \mathrm{~mm}$, i.e., about 2 interactions lengths when using $\lambda=12 \mathrm{~cm}$. We propose to add at the front 20 layers of $2 \mathrm{~mm}$ thick U-plates interleaved with the same type of stainless steel boxes containing TMP. The readout granularity would be $5 \times 5 \mathrm{~cm}^{2}$ in the barrel (supergondolas) for $0.9 \leq y \leq 1.5$ and $10 \times 10 \mathrm{~cm}^{2}$ for $0 \leq y \leq 0.9$, which would decrease to $2.5 \times 2.5 \mathrm{~cm}^{2}$ in the end caps for $y>1.7$ (superbouchons). The 20 layers would be divided into two longitudinal readouts. The electromagnetic calorimeter at $90^{\circ}$ would thus become $120 \mathrm{~mm}$ thick, corresponding to $37 x_{0}$. The total thickness of the U-calorimeter would be 2.3 $\lambda$. The required additions are given in the Summary Table 10, which shows that the total numbers of added towers are 9,650 for the central part and 12,400 for the two end caps. Since there is a twofold longitudinal segmentation, the number of added electronics channels is twice this. 
Table 11

Momentum Precision of Upgraded UAI Detector

\begin{tabular}{|c|c|}
\hline y Region & $\Delta P_{T} / P_{T}{ }^{2} \quad\left(G e V^{-1}\right)$ \\
\hline$\geq 3$ & $1.8 \times 10^{-4}$ \\
\hline $1.75-3$ & $1.3 \times 10^{-4}$ \\
\hline $1.2-1.75$ & $2.1 \times 10^{-4}$ \\
\hline $0.9-1.2$ & $3.6 \times 10^{-4}$ \\
\hline $0-0.9$ & $1.4 \times 10^{-3}$ \\
\hline
\end{tabular}




\section{Iron Calorimeter}

The scintillators are at present located in slots of the $80 \mathrm{~cm}$ thick iron of the central part and $120 \mathrm{~cm}$ of the end caps, which is longitudinally subdivided in $5 \mathrm{~cm}$ plates. There are about $2000 \mathrm{~m}^{2}$ of scintillators in the central part and $1000 \mathrm{~m}^{2}$ in the two end caps. Thus $3000 \mathrm{~m}^{2}$ of gas tubes with readout pads of $30 \times 30 \mathrm{~cm}^{2}$ for the central part and $15 \times 15 \mathrm{~cm}^{2}$ for the end caps have to be added. We foresee a two-fold longitudinal segmentation.

The total number of interaction lengths for a particle at $90^{\circ}$ would eventually be $2.3 \lambda(U)+4.7 \lambda(\mathrm{Fe}) \cong 7 \lambda$ in the central part $(y \leq 1.5)$ and $2.3 \lambda(U)$ $+7.3 \lambda(\mathrm{Fe})=9.6 \lambda$ in the end caps.

The existing gaps in the iron are $17 \mathrm{~mm}$ thick, so that standard Iarocci tubes, as they are now made in Frascati, would fit.

\section{Muon Toroids}

It is not clear whether the addition of muon toroids which would increase substantially the cost of the UAl upgrade is sensible. However, to make the UAl detector more comparable to COF and 00 , we have considered external toroids, as shown in Fig. 10, in an arrangement very similar to that proposed for the $D$ Of upgrade. In our channel count in the table, we assume similar 10 $\mathrm{cm}$ tubes just as in $D \varnothing$. We further assume that the tubes are arranged in 3-layer decks with one deck before the toroid, one (two) decks internally for the central (end) toroids and one deck outside the iron. With this arrangement the numbers of muon tube channels are the same as for the upgraded Dø. 


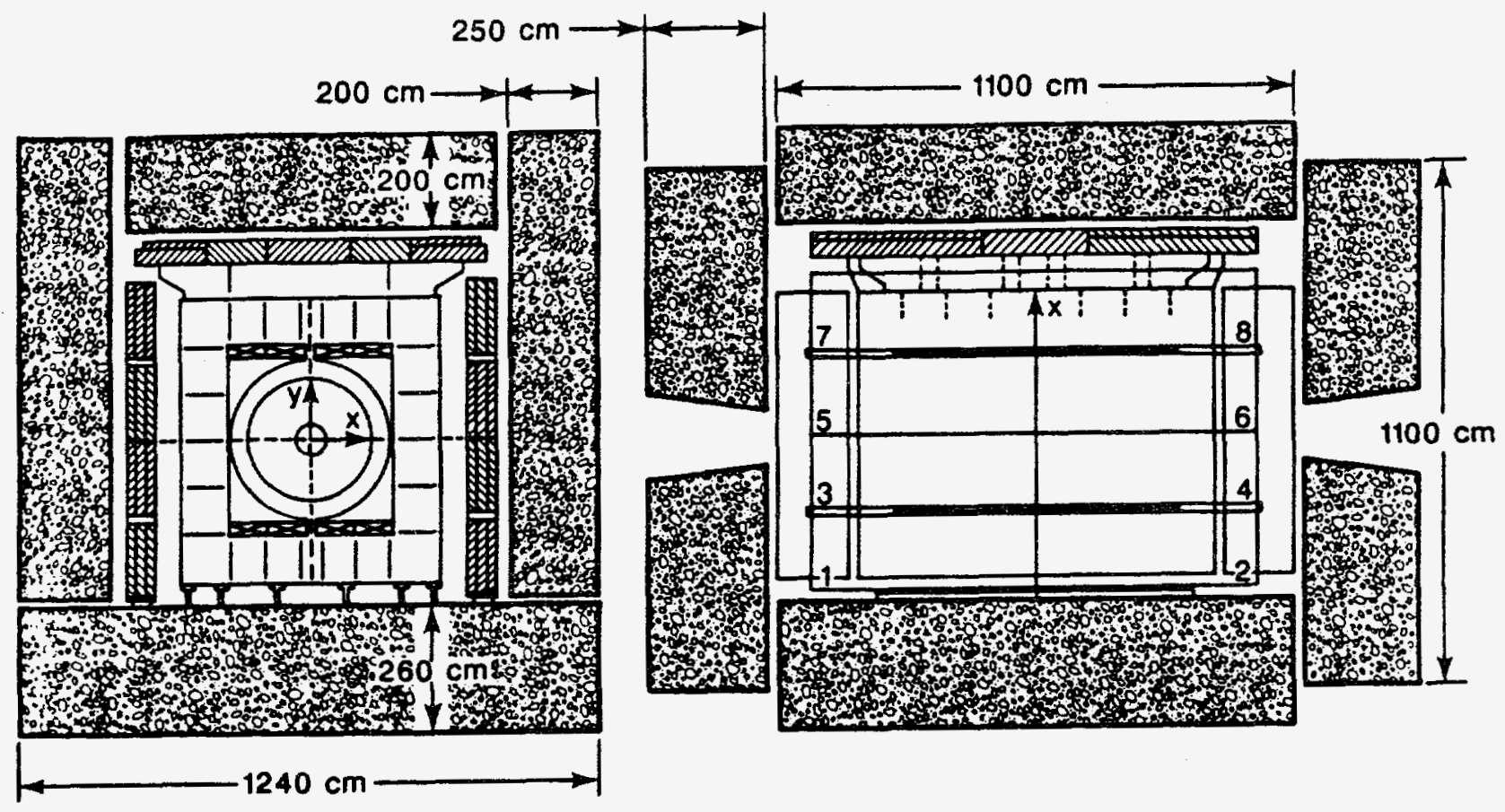

Fig. 10. Cross-section and elevation views of the proposed toroids. 

Appendix 1

Initial Detector Complement Advisory Panel

Dr. U. Amaldi

Professor C. Baltay

Professor M. Gilchriese

Professor F. Gilman

Professor P. Grannis

Professor $H$. Hofer

Professor M. Koshiba

Professor S. Mori

Professor G. Trilling, Chairman

Professor $H$. Williams

Professor $B$. Winstein

Professor M. Witherell
CERN, Switzerland and University of Milan, Italy

Columbia University

Cornell University

SLAC

State University of New York, Stony Brook

Eidg. Technische Hochschule, Switzerland

University of Tokyo, Japan

University of Tsukuba, J apan

Lawrence Berkeley Laboratory

University of Pennsylvania

University of Chicago

University of California, Santa Barbara 


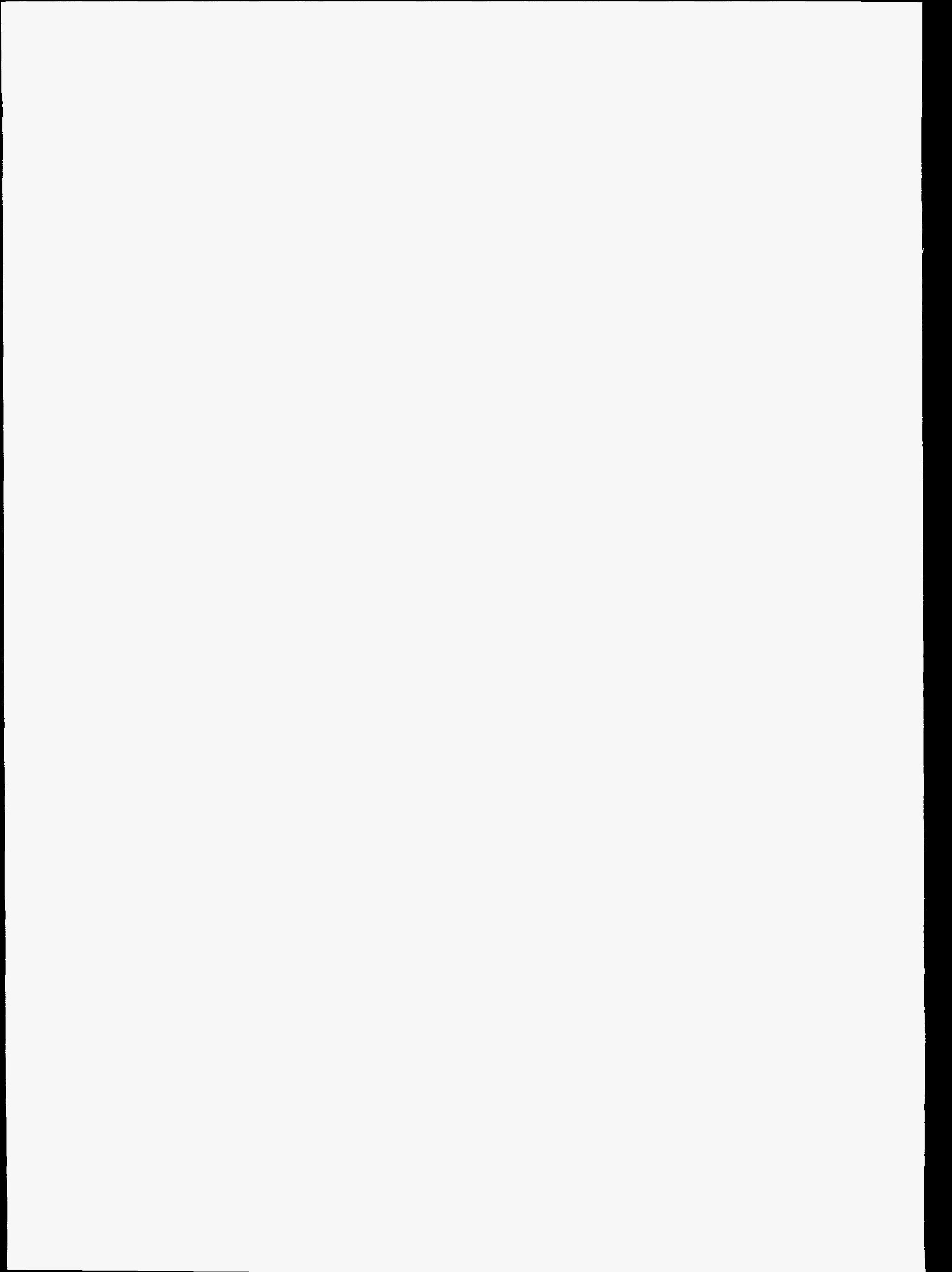




\section{ABSTRACT}

We have built an $80 \mathrm{~cm}$ long multiwire drift tube with 128 ndependent anodes, mounted within a light hexagonal carbon-fibre nvelope $3 \mathrm{~cm}$ in diameter. Several modules can be assembled together to puild a vertex detector suitable for collider experiments. Measurements on prototypes and simulations have provided a single-hit accuracy below $00 \mu \mathrm{m}$ and a two-track resolution around $300 \mu \mathrm{m}$.

An obvious way to improve the rate capability and two-track esolution of a multiwire proportional chamber, given its intrinsic imitations, is to increase the density of sense wires in order to reduce the lux of radiation on each element $[1,2]$. We are building a multidrift ertex detector composed of many independent modules each with the ollowing characteristics (see Fig. 1): 128 independent drift cells are packed within a carbon-fibre supporting tube, also of hexagonal shape, hat constitutes the gas envelope. Each sensitive cell consists of an anode vire, $30 \mu \mathrm{m}$ in diameter, centred in a hexagonal structure $1 \mathrm{~mm}$ in radius vith $70 \mu \mathrm{m}$ cathode wires at the corners. The wires are strung between wo fibre-glass hexagonal end plates with an outer dimension of $30 \mathrm{~mm}$, ind perforated on a numeric drilling machine. Each wire is soldered in he groove of a high-precision metal pin inserted in the holes of the upporting plate (Fig. 2); all cathode wires are connected together, and perate at a negative high voltage, while the anodes are individually vired to the amplifier and discriminator circuits. Although for the first prototype we are using conventional, thick-film hybrid electronics lements, a large-scale integration is envisaged to reduce the volume and ost of the hardwate. We intend to profit from the effort already avested for the needs of the microstrip silicon detectors, where similar problems of packaging density are met. A simplified delay line readout erializing large groups of wires is also being tried.

The prototype drift tube is $80 \mathrm{~cm}$ long, adapied to use as a vertex etector around a collider; many cubes can indeed be closely packed in a

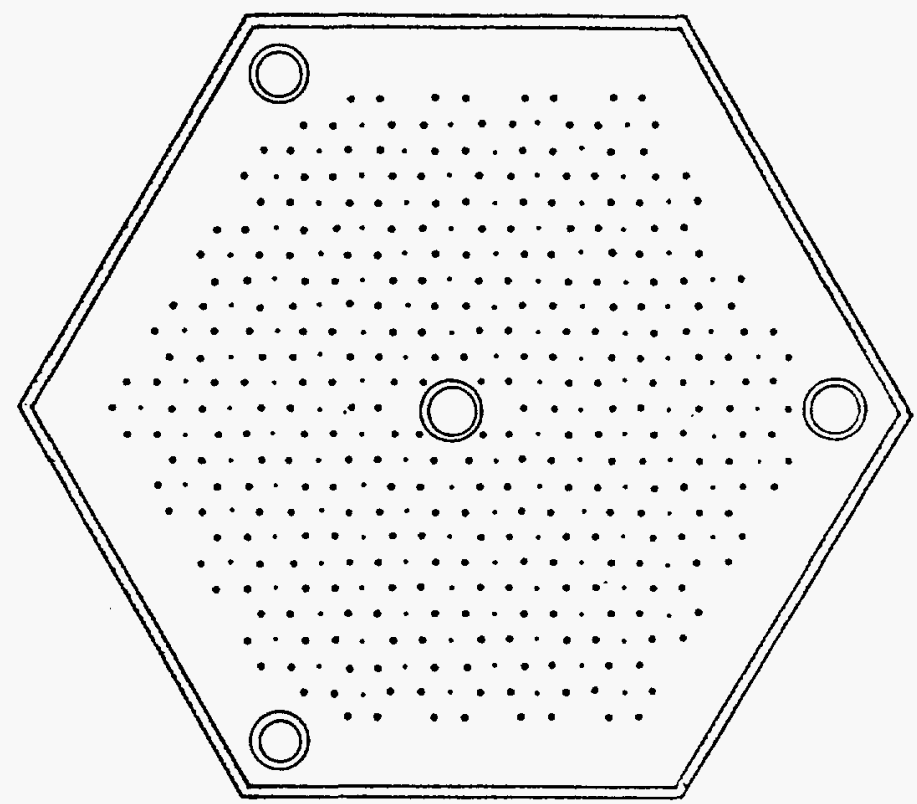

Fig. I Schematic of the multidrift detector module. 123 independent drift cells. each realized with an anode wire centred on a hexagonal pattern of cathodes, are packed in a carbon-fibre hexagonal envelope, $30 \mathrm{~mm}$ in width.

cylindrical geometry. A contact with the anode wires is available at both ends of the detector, to allow a longitudinal coordinate determination through current division; in this case the wires should be of the resistive type. We have also developed a cheap multi-connector using soft conducting pads inset in a printed-circuit board drilled with a hole matrix that repeats the layout of the anode pins. Figures 3 and 4 show the wiring and assembly of the multidrift module. Wires are first pulled through the

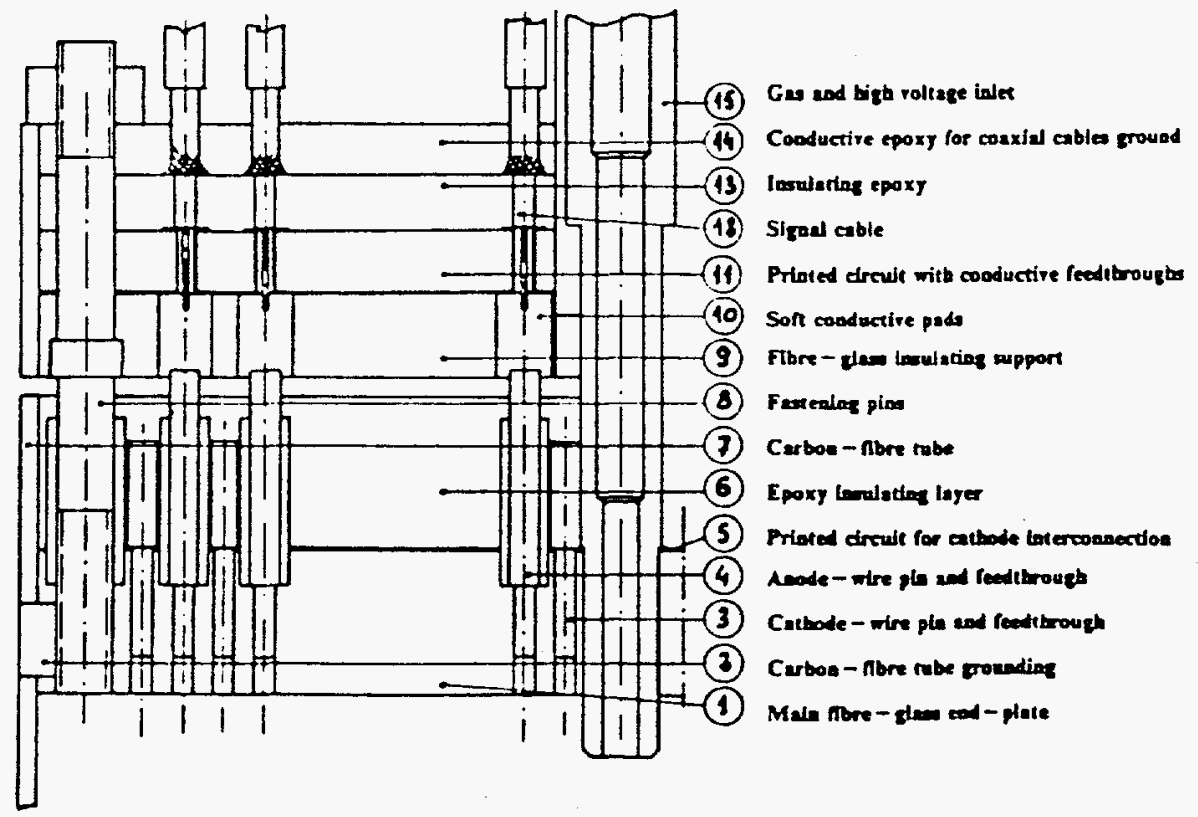

Mg. 2 Detailed cross-section of the end plate and connector assembly. 


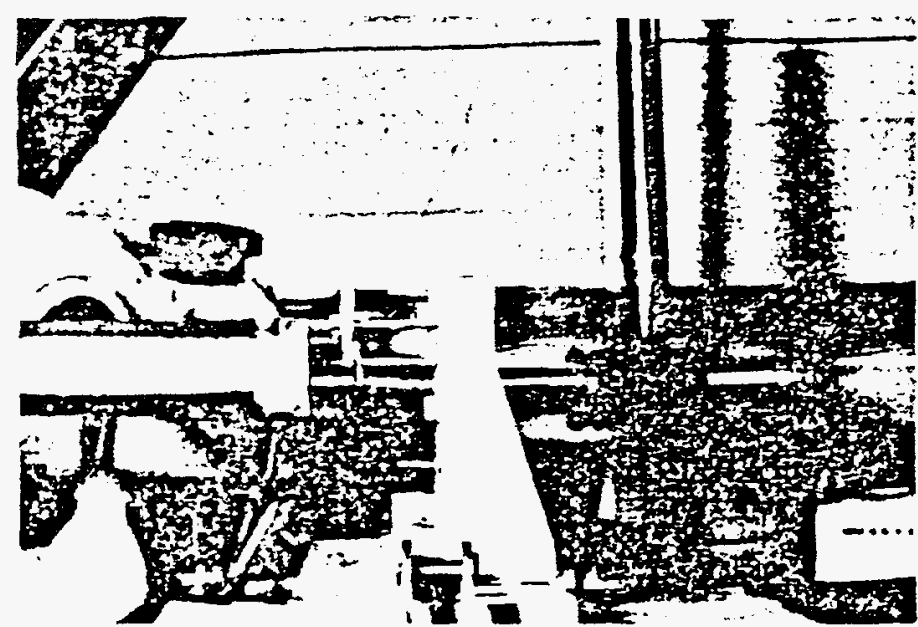

Flg. 3 Final phase of assembly of a multidrift moduie. All wires are stretched and fastened to the end plates: the carbon-fibre tube is ready to be slid over the wires, after removal of the plate's support.

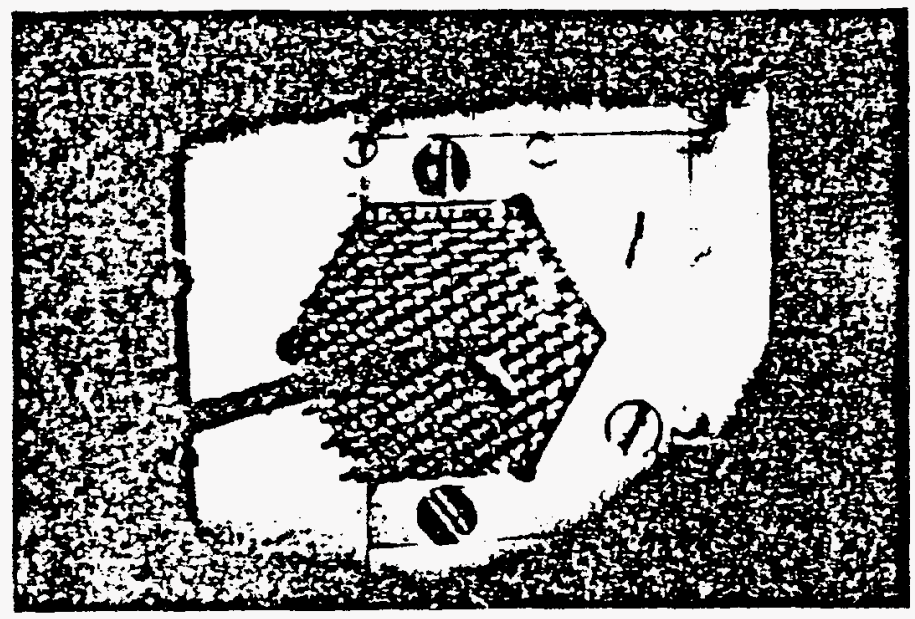

Elg. 4 Detal of the temporary support of the end plate during wiring.

end plates. which are fixed to the bench; the supporting cylinders are inserted and the wires soldered under tension. Once all wires have been strung, the end plates are fixed to central rods, one of which holds the hexagonlal carbon-fibre supporting tube, and freed from their supports; the tube is then slid in and epoxied at the two ends on the plates. The gas inlet and high-voltage connection are provided through a central pin on each side.

The first measurements realized with a 32 -wire prototype in a minimum ioming test beam confirm the relative ease of operating the counter in many gas mixtures, although to avoid edge discharge we prefer to use small fractions of quenchers in argon so as to limit the absolute working voltage. Detection efficiencies close to $100 \%$ and localization accuracies of $100 \mu \mathrm{m}$ r.m.s. on each wire have been recorded.

A typical reconstructed event is represented in Fig. 5; the display shows also the coordinate of the track measured with a conventional high-accuracy drift chamber used as reference. The space-time relationship of a single wire for a uniformiy spread beam, measured by comparison with the drift chamber, is shown in Fig. 6. The pattern exhibits the typical right-left symmetry of a drift cell around the anode wire; the linearity of the response is rather good, and the width of the distribution of the scattered points provides the localization accuracy of the cell, having an $\mathrm{r} . \mathrm{m}$.s. of around $100 \mu \mathrm{m}$ (the maximum drift length is $1 \mathrm{~mm}$ on each side).

One interesting way of operating the counter is to use an electronegative gas mixture, such as a large proportion of freon in a

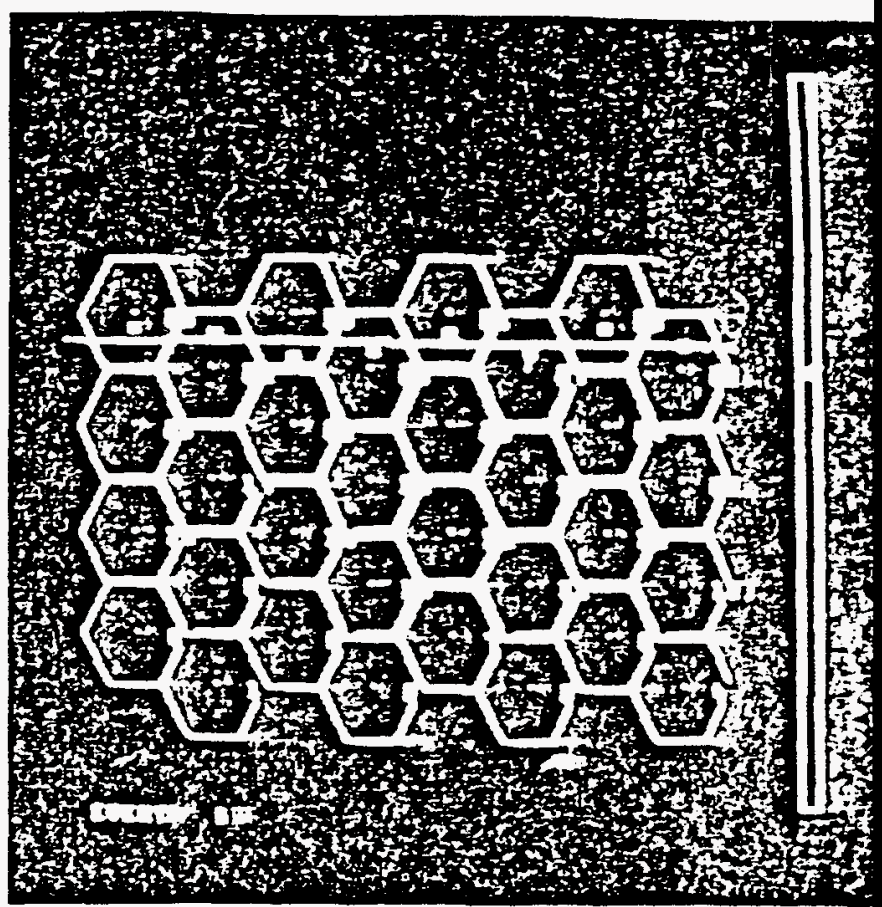

Flg. 5 Computer display on one track, recorded with a 32 wire prototyp multidrift detector. The coordinate of the track provided by a referenc high-accuracy drift chamber is also shown.

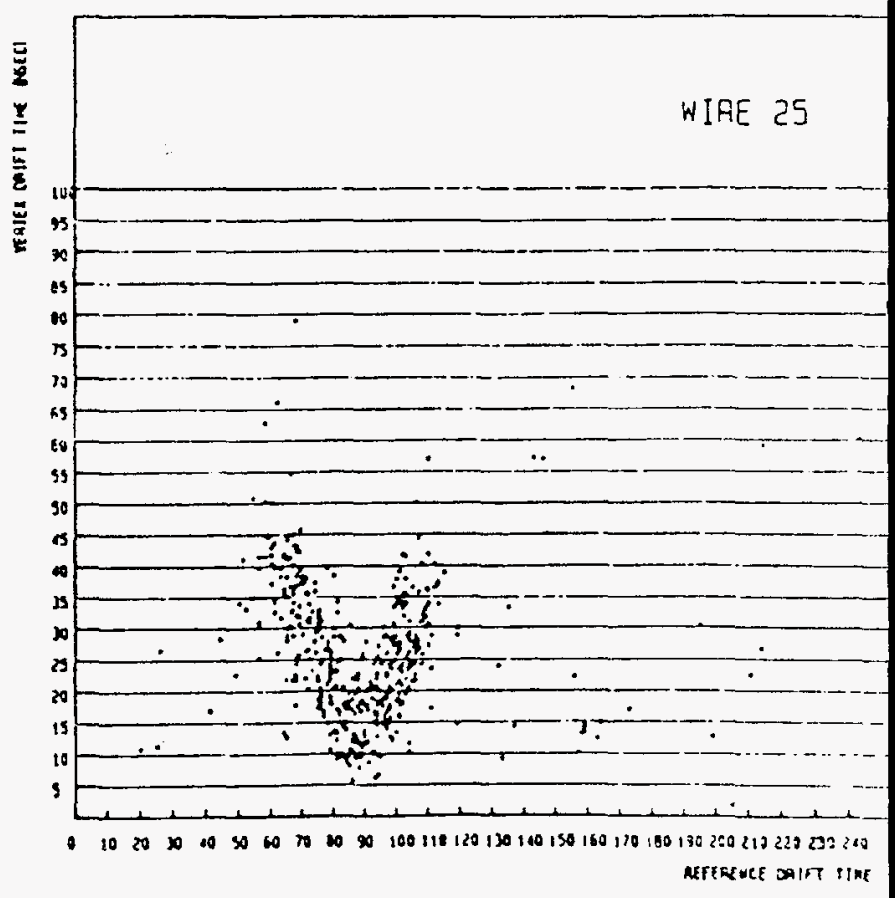

F1g. 6 Space-time relationship in a single wire of the multidrift detecto obtained in comparison with the coordinate provided by the reference drit chamber (horizontal coordinate). The good linearity of response and it localization accuracy (about $100 \mathrm{~mm}$ r.m.s.) are apparent. Gas filling: argon isobutane-methylal (70-25-5).

normal gas. In this case the efficient region of detection can be restricted to a narrow cylinder around each anode; for $20 \%$ freon in argonisobutane the sensitive region has about $200 \mu \mathrm{m}$ FWHM. A space-tim. correlation measured on a wire in these conditions, always with referenc. to an external drift chamber, is shown in Fig. $7:$ all points cluster withit a small region around the anode wire, as expected. The counser can the be used as a purely digital one, each wire providing a signal only fo 


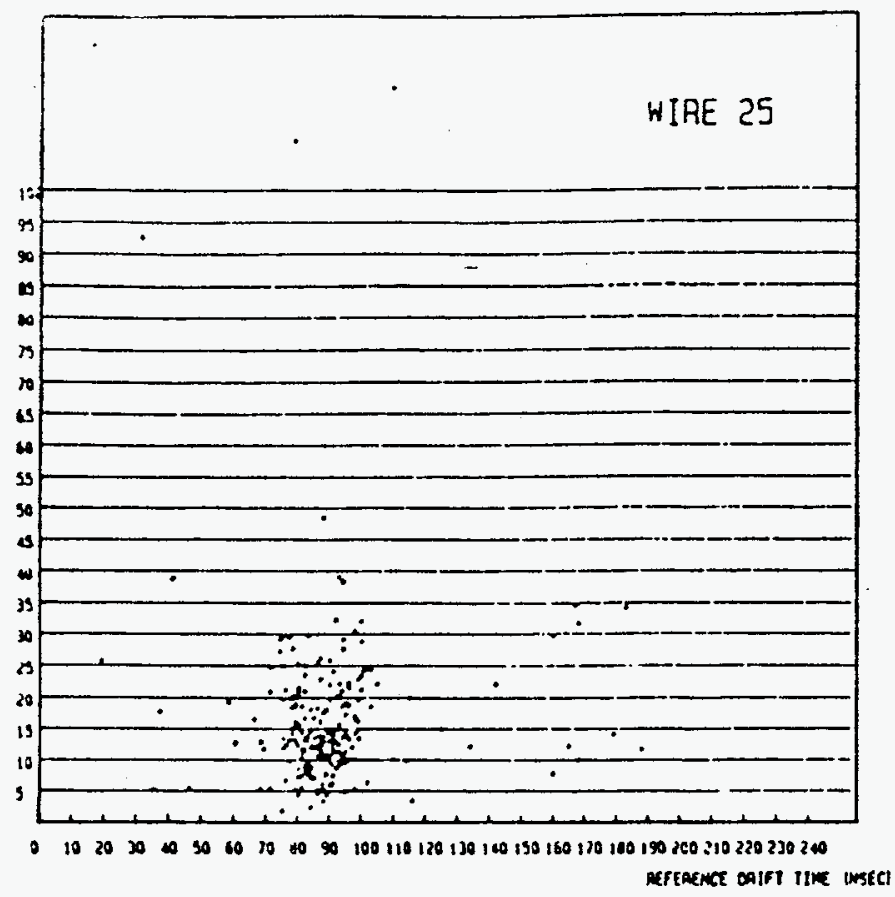

Fig. 7 Space-time relationship for a single wire of the detector filled with an electronegative gas mixture, argon-isobutane-freon (70-10-20). The FWHM of the efficient region around the wire is $200 \mu \mathrm{m}$.

tracks within the quoted region. This mode of operation allows the highest flux capability (because of the reduced efficiency) and the use of a simpler pattern electronics; the number of measured points on each track is of course reduced.

To anaiyse the resolution properties of the device and optimize the pattern recognition, we have developed a rather detailed generation program for Monte Carlo events that takes into account the primary and total ionization statistics and the drift and diffusion of the electrons in the cells of the detector. The simulation indicates that an average reconstruction accuracy of $70 \mu \mathrm{m}$ r.m.s. should be achievable on each cell: in the complete tube, a single track is measured on an average a dozen times with the quoted accuracy. The time resolution of the counter is about $30 \mathrm{~ns}$. corresponding to the maximum drift time.

For two near tracks of course only the coordinate closest to each wire is measured; the geometry is such, however, that on an average each Irack will still be recorded on alternate wires and can be reconstructed, although on a reduced number of wires (see the example in Fig. 8 generated and reconstructed with the described program). We expect indeed 3 two-track resolution of two or three hundred microns, somewhat depending on the sophistication of the software reconstruction

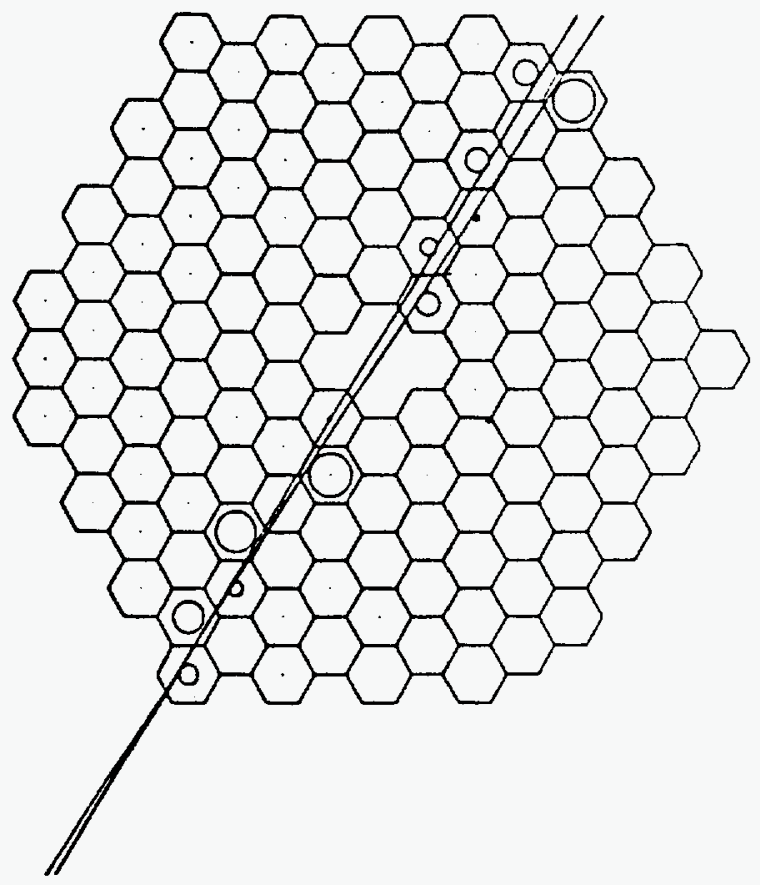

Fig. 8 Monte Carlo simulation of two tracks detected by a multidrifi module Circles indicate the measured dtift times, and the straight lines the reconstructed iracks.

algorithm. For dense concentrations of tracks, as in jets or for calorimetry applications, the envelope of the cluster of tracks should stit! be well determined.

The success of this approach to the realization of a high-rate, high-resolution detector will depend, of course, on the finding of cheap and reliable construction techniques, as well as on the development of a high-density electronics.

\section{References}

(II R. Bouclier, G. Charpak and F. Sauli, CERN EP Internal Report 84-03 (1984).

[2] F.Sauli, New detectors for high-intensity beams, preprint CERNEP/85-134 (presented at the Meeting on the Future of Medium- and High-Energy Physics in Switzerland, Les Rasses, Switzerland, 17-13 May (985). 


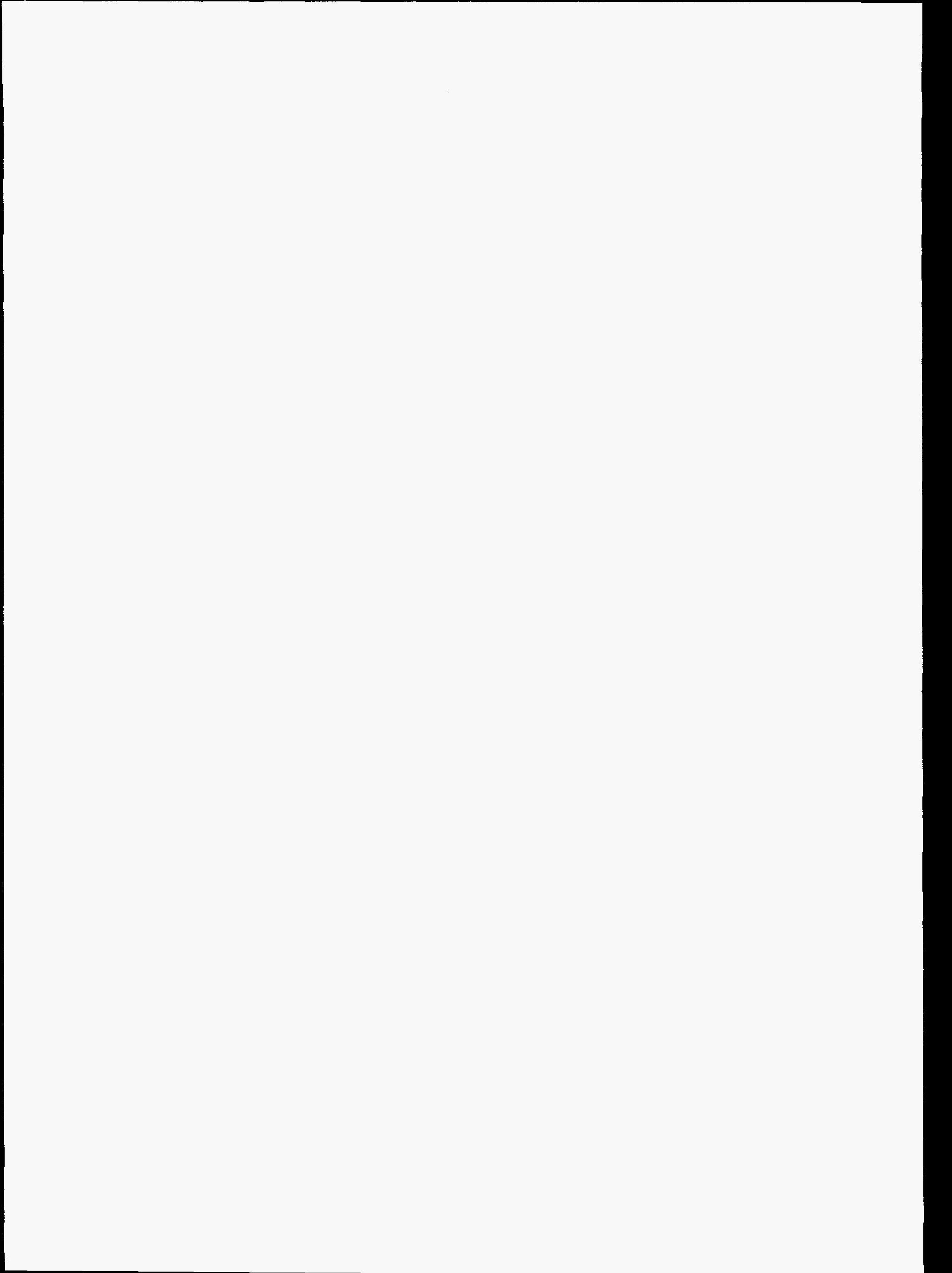




\section{Report of the Detector Cost Evaluation Panel}
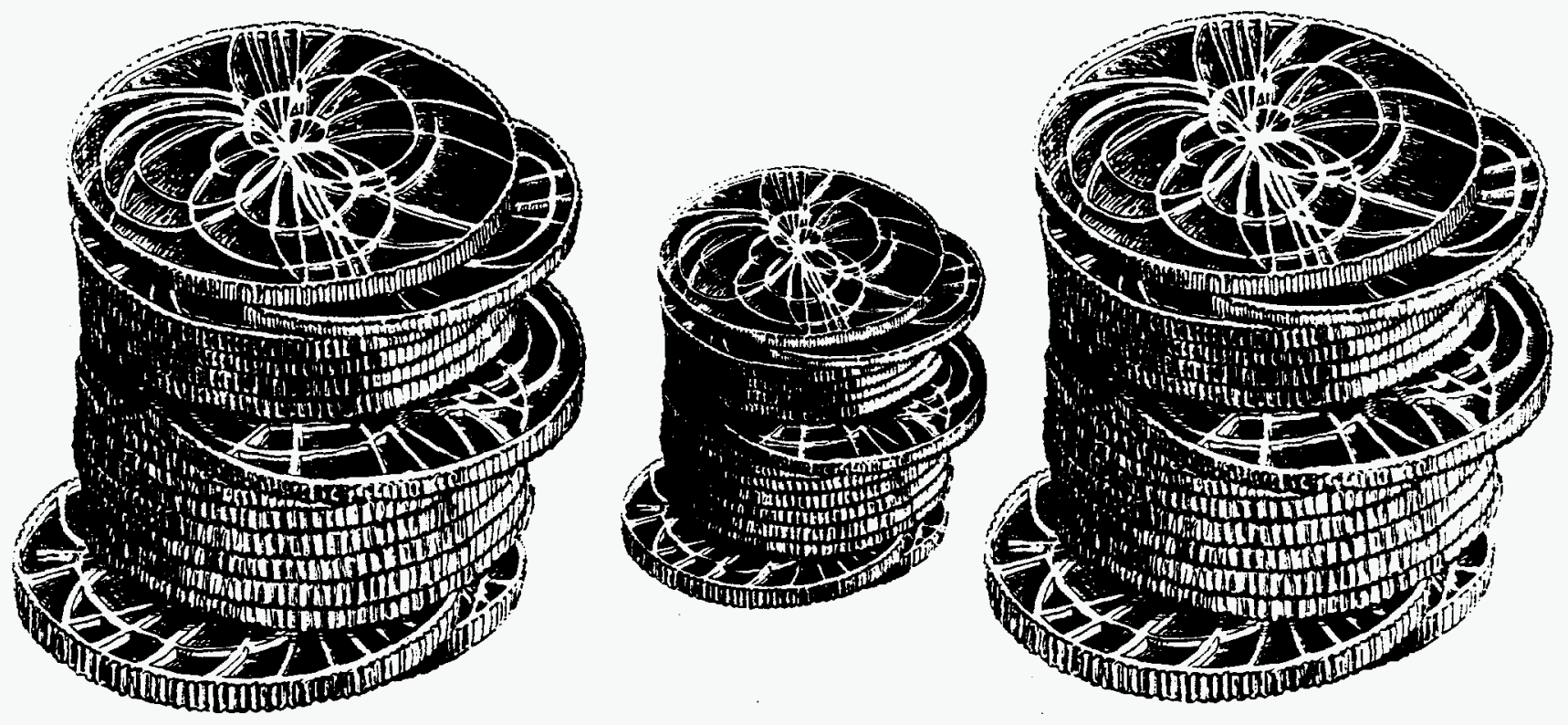

May 1986 


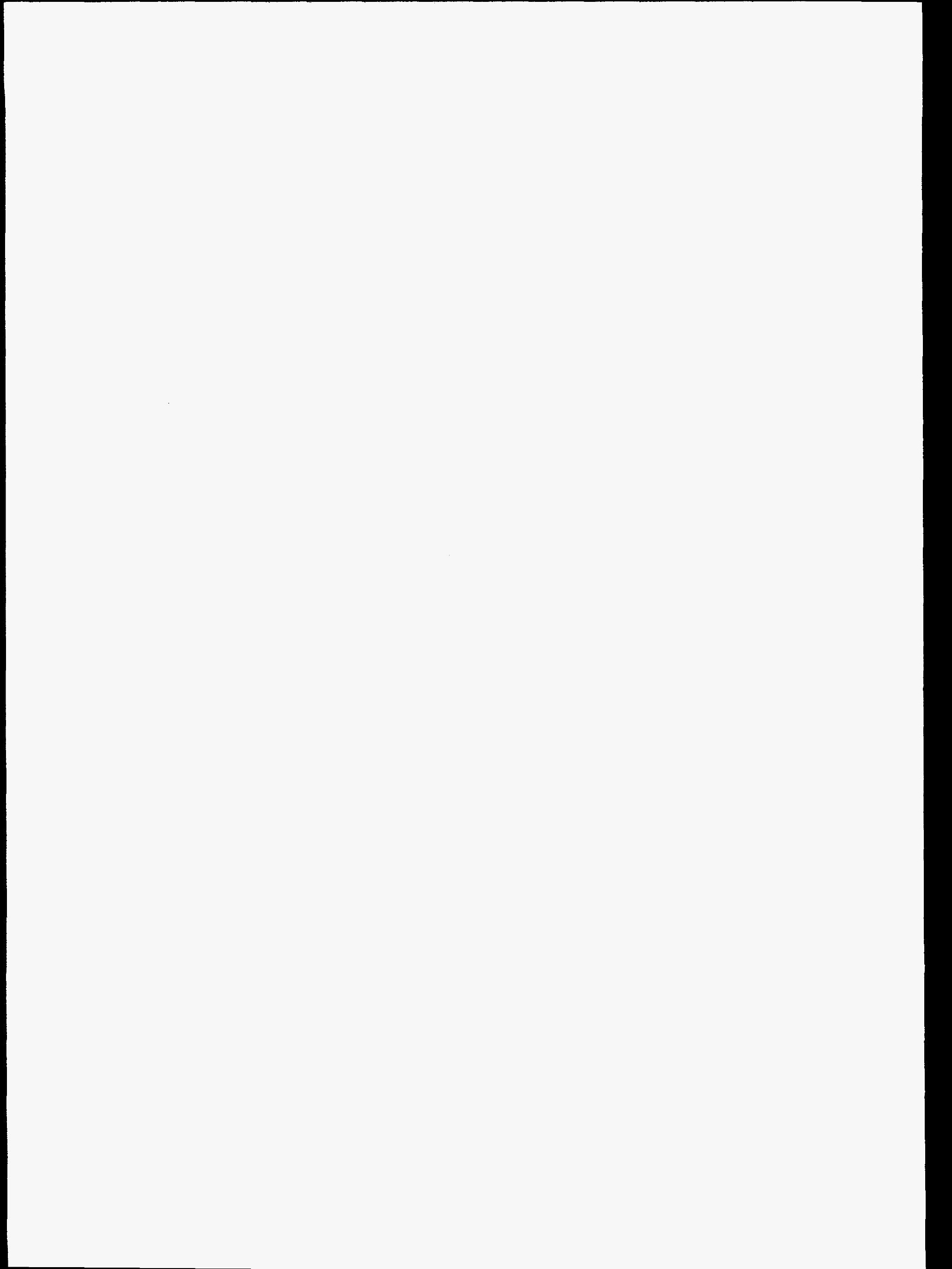




\section{INTRODUCTION}

The Detector Cost Evaluation Panel was charged by Maury Tigner to provide the SSC Central Design Group with an estimate of the probable cost of the initial detector complement at the SSC. The membership of this panel is given in Appendix $A$. The specific charge to the panel was:

"Using the recommendations of the Detector Cost Model Advisory Panel as the picture of the initial detector set at the SSC, the Detector Cost Evaluation Panel shall estimate the cost of constructing the first SSC detectors. The panel should use the cost data accumulated in the course of construction of CDF, SLD, DO, L3, and other recent big detectors as the basis for its cost estimates. The panel should use its best judgement in estimating possible future decrease (or increase) in the costs of various components and sub-systems. The cost estimates should allow for all the electronics and on-line computers necessary for each detector, but not the off-line computing facilities that may be necessary for subsequent processing of the data.

"The panel should assume that the construction of the detectors should be timed so that they could be in place for the first pp collisions in 1994. The costs should be expressed in FY86 \$."

As discussed below, there is considerable uncertainty in projecting costs of the potential SSC detectors described in the report of the Detector Cost Model Advisory Panel (DCMAP) because of uncertainties in scaling costs of current detectors, unknown advances in detector components resulting from future R\&D, and because the detectors considered represent typical desired parameters rather than consistent engineering designs. There is significant elasticity between cost and performance that comes into play when actual designs are carried out. Nevertheless, we believe that these cost estimates are useful in setting the scale of support needed for SSC detectors.

We considered the engineering, design, inspection, and administration (EDIA) needs of the detectors studied. This component was added to the costs scaled directly from the parameters given in the DCMAP report. 
Thus, the estimates presented here represent the total equipment funds needed for the DCMAP detectors as if the detectors were to be built in a laboratory environment like SLAC or Fermilab under practices established for CDF, D $\varnothing$, and SLD. These equipment costs include EDIA, M\&S, local shop services, fabrication labor and overhead on laboratory labor. The estimates do not cover escalation and operating, or pre-operating costs, which include costs of R\&D, software, physicists and general laboratory overhead.

\section{CONCLUSIONS AND RECOMMENDATIONS}

From the cost assumptions and methods described below, the estimated costs for the detector scenarios given in the DCMAP report, including EDIA, may be summarized as:

1. A new $4 \pi$ Magnetic Detector $\$ 290 M-\$ 334 M$

2. A Spectrometer for High Energy Muons $\$ 159 M-\$ 174 M$

3. An Upgraded Existing Detector

$\$ 90 M-\$ 125 M$ Additional Forward/Intermediate Detector $\$ 95 \mathrm{M}-\$ 102 \mathrm{M}$

4. Specialized Detectors (Total Cross Section, etc.)

$<\$ 20 M$

5. Program Contingency

$\$ 175 \mathrm{M}$

The indicated ranges are based on the variance in the number of electronics channels given in the DCMAP report, and on the variations between individual detectors studied within a given category. It is our judgement that these ranges should be increased by $\pm 15 \%$ to account for the elasticity in initial scope of the detectors and uncertainties in cost projections. This yields a total cost without the program contingency in the range $\$ 558 M-\$ 865 M$.

The expected costs of special detectors for measuring the total cross section, elastic scattering, etc. are very small compared with the general purpose detectors and, therefore, cost estimates were not calculated explicitly.

The estimates given above for the large detectors are best estimates of the full equipment funds needed, but explicitly do not include contingency. Contingency will be needed to cover errors in the estimating process and to cover items which are forgotten. Our experience indicates that an average contingency 
of 30-35\% is appropriate for a complete, cost-optimized design. We believe it inappropriate to suggest a definite contingency for the cost-scaling errors of each detector because there is comparable uncertainty in the required scope of these detectors. However, we extended the ranges of costs by $\pm 15 \%$, roughly the amount these estimates change if the number of detector channels is halved, to account for these uncertainties. To plan for the items that are inevitably left out of initial cost estimates of optimized designs, we suggest that an overall program contingency of about $25 \%$ should be applied to the full set of detectors. For the DCMAP complement of detectors, this program contingency amounts to $\$ 175 \mathrm{M}$. Since the DCMAP plan is only a guide, the actual contingency assigned to SSC detectors should be evaluated when the real detector designs are known.

We therefore conclude that the total detector costs, including EDIA and contingency, for the program outlined in the DCMAP report will $l$ ie in the range $\$ 733 M-\$ 1040 M$. It should be noted that the DCMAP detector plan is aggressive and that a rich physics program for the initial period of SSC operations probably could be achieved with a somewhat different mix of detectors that may reduce the overall cost.

After considering these cost estimates, we feel that the following recommendations should be considered in planning for detectors at the SSC:

1. Detector R\&D should be encouraged and supported promptly. The times scales for large detector construction and SSC construction imply that only a few years are available to conduct the highly leveraged R\&D that will affect the basic detector techniques and costs. This is especially true in the area of electronics where we must achieve the construction of faster electronics at a fraction of today's costs.

Since the R\&D needs are large, every effort should be made to share R\&D on common problems.

2. EDIA efforts should be shared where possible. The estimates in this report assume independent EDIA efforts, but many of these areas overlap. Administration and management structures, such as budget, procurement and personnel services should be shared as well as technical services including, for example, safety, cryogenics, electronics, and software.

3. Detector software should be considered part of the detector construction project and not an operating 
expense. The scope of modern detector software requires professional software engineering and management efforts as well as physicist contributions, and should be included in detector cost estimates. (We have followed traditional practice in this report and have not included software cost estimates). University software contributions should be considered equivalent to hardware contributions.

\section{COST ASSUMPTIONS}

The costs of large detector systems can be divided into a number of categories for the purposes of estimating the scaling laws that should apply to possible SSC detectors. In general, there are costs associated with the overhead of engineering, design and management of each large subsystem, costs proportional to the weight of component systems, costs proportional to the number of wires of electronics channels, and so forth. There is no unique way to determine these costs. We have determined scaling laws and cost coefficients based on experience with existing or soon to be completed detectors. We note that in the general area of electronics, substantial improvements must be made in cost per performance to make our estimates a reality. One may reasonably expect this to occur as a result of research and development and the evolution of industrial expertise in electronics design. We note that estimates for installation costs have been included in subsystem costs, although there are uncertainties resulting from the lack of a specific site for the SSC. Also we have not included transportation costs of the unchanged components of the upgraded detectors. Cost estimates for some of the components of the precision muon spectrometer (magnet, hadron calorimetry and muon tracking) were obtained from the L3 group and costs for the remaining components (electronics, trigger, on I ine computer and EDIA) were obtained from the most recent DOE budget reviews of CDF, DQ, and SLD as described below. Our assumptions about cost estimates for all components are given in Appendix B, Table 11 and are discussed below.

\section{Fixed Costs}

We have assumed that there are a number of fixed costs to be contained in some or all of the detectors described in DCMAP. These include online computing facilities located at the experiments, liquid Helium cryogenic systems and I iquid Argon cryogenic systems. In making our estimates we have ignored small differences in the scale of such systems that will be appropriate to each detector. A major component of the cost estimates are engineering, design, inspection and administration (EDIA) costs for each major subsystem. Wo have estimated these costs in great 
big lumps (GBL) which correspond to six man years of engineering, eight man years of design and 10 man years of inspection, administration, etc. For each major subsystem we have estimated the total number of GBL required.

Iron Costs

A major fraction of the weight of the large detectors described in DCMAP results from iron used for muon detection. The cost of iron, magnetizing coils, support, installation and transporters, was estimated from the costs of similar systems in CDF, DQ and SLD.

Calorimetry Costs

There are many types of calorimetry contained in the detectors described in DCMAP. We have estimated these costs for each type on a per ton basis from similar systems in CDF, D $\varnothing$ and SLD. Although the calorimeter systems contained in the large $4 \pi$ detector options are substantially larger than present or planned systems, we have not included any cost reduction as a result of the larger scale of these systems.

\section{Magnet Costs}

The feasibility and cost of constructing the large superconducting magnets in the Model A and Model B 4x detectors has recently been examined by the Task Force on Detector R\&D for the SSC . They conclude that it is feasible to construct these magnets. We have used their cost estimates which were based, in part, on extrapolations from existing magnets (e.g.CDF) or magnets now under construction. Cost estimates of large aluminum conductor magnets of the $L 3$ type are based on the L3 experience.

\section{Tracking Detectors}

We have made cost estimates for three different types of tracking devices; central and planar wire chambers, muon chambers and silicon strip detectors. There are substantial uncertainties in the required performance and characteristics of silicon detectors for the SSC. As a result we have chosen to apply a single cost to such silicon tracking systems including the readout electronics, rather than apply a cost per $\mathrm{cm}^{2}$, for example, or by readout channel. It is now clear that electronic readout of silicon detectors must be integrated with the actual silicon detector and therefore cannot be easily separated for costing purposes. 
There are somewhat smaller uncertainties in the scale required for wire tracking chambers for the SSC. Modest advances in technology, such as the use of fast drift velocity gases, could reduce the required number of wires by a factor of 2-3 over the numbers given in the DCMAP report. Also the tracking systems described in this report contain 10-20 times more active wires or other readout elements than current devices at existing colliders. At present the cost of mechanical supports (e.g. endplates) is a major, even dominant, fraction of the total cost of central tracking devices. For the very large systems described in the DCMAP report, this may not be the case as a result of the large number of wires in such systems. Also for such large systems it is reasonable to assume that automated wire stringing mechanisms or modular construction techniques will reduce the effective cost per wire over existing chambers. Modular methods, for example, have been used for many years in the construction of planar chambers. We therefore have chosen to estimate the cost of wire tracking chambers based on a single cost per wire.

We have estimated the cost of muon chambers in two different ways; scaling by the area coverage of the chambers and by cost per wire as indicated in the previous paragraph. The latter method was used for the Model $A$ and $B$ detectors and the former for all others. The two methods were chosen to conform with the DCMAP tables. The cost per area was estimated from the CDF and DQ experience.

\section{Electronics and Trigger}

The cost estimate for electronics is necessarily uncertain as a result of the limited detailed information on electronics requirements at the SSC and uncertainties in future trends in electronics design. Our estimate is based on three components of the electronics cost; front-end electronics (including preamplifiers), connectors, cables and installation; digitizing, correction and $f i l t e r i n g$ electronics; and an average cost per channel for trigger elements. Although there will, no doubt, be some differences in the actual cost of the electronics for each subsystem (inner tracking, calorimetry, muon chambers, etc.) and as a result of different luminosity requirements, at present we do not believe there is sufficient information to reliably estimate these differences and have chosen to give one average cost per channel of $\$ 120$.

The front end cost is estimated to be $\$ 50$. We have assumed a full back-end channel would cost $\$ 500$, but that very fast time domain multiplexing would let it be shared by 10 front end channels, for an average cost of $\$ 50$ per channel. Finally, based 
primarily on CDF experience, we have estimated that a channel of trigger processing electronics would be $\$ 200$, but that on ly $10 \%$ of all signal channels would be included in the trigger. This yields an average cost of $\$ 20$ per channel for a total of $\$ 120$ per channel.

\section{COST ESTIMATES}

Spread sheets are presented in Appendix B for each of the detector summary Tables given in the DCMAP Report. The costs are broken down by hardware systems, electronics, onl ine computers and EDIA by system. For each line, the system costed, the costing unit, and the unit cost are given. Also given is a code for the costing unit for cross referencing to a complete listing of such costs given in Table B-11. We also tabulate subsystem (tracking, calorimetry, muon, electronics, etc.) costs and the percentage of the total cost. We note that the electronics costs given in the spread sheets for the Model A and B detectors have assumed a mean number of channels rather than the range given in the corresponding tables in the DCMAP report. The total costs for the detectors are summarized in Table B-10, along with the variance in total cost assuming a $\pm 10 \%$ change in the total - lectronic channel count, and the percentage breakdown by subsystem.

\section{GENERAL COMMENTS}

We have gone through an exercise of costing the detectors described in the DCMAP. There is a lot to be learned from the detectors and their estimated costs, but these lessons should be used with extreme caution. We offer the following comments:

1) No attempts have been made to optimize the detectors, such that one would get the maximum physics for the minimum costs.

This process is one which naturally occurs in the early stages of detector design, as the group more sharply focuses its goals on physics. We believe for example that the level of uncertainties in our estimating is probably of the same level as reductions in electronic channel counts, which could occur with new readout techniques.

2) The major cost items for the detectors are not surprisingly in calorimetry, iron, and electronics. Without a detailed design it is hard to see how one can much affect the costs of the detector without changes in scope of these systems. EDIA tends to be 15-20\% of the total cost which again is set by the scope. 
3) No attempt has been made to estimate economies in scale which could be realized in large systems which might, for example, draw on industrial techniques not available to today's detectors.

There are of course no such economies in purchase of iron.

4) We have been extremely aggressive in assuming a new generation of electronics and trigger. Our estimate of $\$ 120 /$ channel is based on pipeline handling of enormous amounts of data. Changes of $30 \%$ in these costs will have large impact on the overall detector cost.

5) Three upgraded detectors have been costed, which cost somewhat more than the base costs of the original detectors. No costs have been calculated for moving the retained portions of these existing detectors. These schemes will of course be closely scrutinized to seo if in fact it makes sense to do such upgrades.

6) Uranium calorimetry figures in many detectors. Depleted uranium scrap is now available thru DOE at Oak Ridge at a cost of $\sim \$ 1 / l b .$, with 420 tons available a year. For SSC needs one might have to process greensalt at a cost of $\$ 6 / / \mathrm{b}$. This could add \$40M to the cost of Model B, for example. It should a lso be noted that the costs for liquid argon calorimetry are based on the cost estimates for the large systems being built for $D \phi$ and SLD. The accuracy of these estimates awaits the completion of the construction.

7) The current detector crop (DD, CDF, SLD and UA1) have benefited from drawing on the resources available at operating laboratories. These include technical and administrative support which is absorbed by the lab and can cost several million per year. 
Costs of a $4 \pi$ detector, similar to Model A considered by DCMAP, were estimated in the proceedings of the 1984 DPF Summer Study. In the table below, we compare these estimates. Trigger costs for Model A were extracted from the overall electronics estimate according to the $\$ 20 /$ channel figure discussed above.

\section{System}

Tracking

Precision Calorimetry

Iron Calorimetry

Solenoid Magnet

Muon System

Elect. Tracking

Elect. Calorimetry

Elect. Muon

Trigger

Online Computing EDIA

TOTAL

\author{
Model A \\ $\$ 14.0 \mathrm{M}$ \\ 50.5 \\ 37.3 \\ 12.5 \\ 45.5 \\ 25.0 \\ 48.6 \\ 16.4 \\ 18.0 \\ 5.0 \\ 43.0
}

$\$ 315.8 \mathrm{M}$
Snowmass 84 (SCD)

$\$ 11.6 \mathrm{M}$

73.7

20.4

19.4

41.5

17.2

8.3

8.0

5.0

5.0

0.0

$\$ 210.1 \mathrm{M}$

The biggest difference between the two estimates is EDIA, which was neglected in the Snowmass study. Electronics costs are also higher in this study. The two reasons for this are the factor of three larger number of total channels given by DCMAP, and the larger average cost per channel of electronics assumed here. It is our judgement that the cost per channel assumed by the Snowmass study in the critical area of calorimetry and trigger are probably too low. The uranium costs assumed in this report reflect recent progress in procuring and rolling uranium as discussed above. The other areas are in reasonable agreement. Note that the Snowmass 84 figures do not include contingency.

The Snowmass report assumed an overall $25 \%$ contingency, or $\$ 52.4 \mathrm{M}$ for the SCD detector. 


\section{APPENDIX A}

SSC Detector Cost Evaluation Panel

Roy Schwitters, Chairman

Department of Physics

Harvard University

Cambridge, MA 02138

Michael Marx

Department of Physics

SUNY

Stony Brook, NY 11794

Martin Breidenbach

SLAC

P.0. Box 4349

Stanford, CA 94305

M.G.D. Gilchriese

Department of Physics

Cornell University

Ithaca, NY 14853 


\section{APPENDIX B}

Detector Spread Sheets

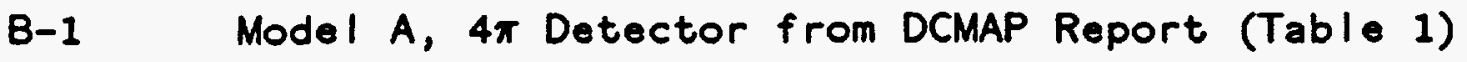

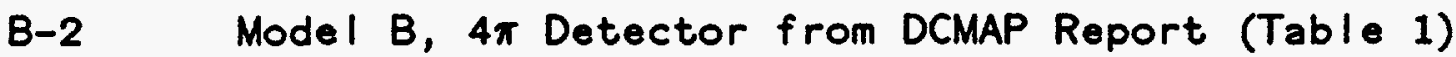

B-3 Precision Muon Spectrometer from DCMAP Report (Table 3) note: cost coefficients based on report prepared by L-3 collaboration.

B-4 Iron Muon Spectrometer from DCMAP Report (Table 4)

B-5 Forward Detector - Upgrade Model from DCMAP Report (Table 5)

B-6 Intermediate Detector - Upgrade Model from DCMAP Report (Table 5)

B-7 CDF Upgrade from DCMAP Report (Table 6)

B-8 Dø Upgrade from DCMAP Report (Table 8)

B-9 UA-1 Upgrade from DCMAP Report (Table 10)

B-10 Summary of total detector costs and breakdown by system

B-11 Cost assumptions for spread sheets 
MOdel a (TABle 1)

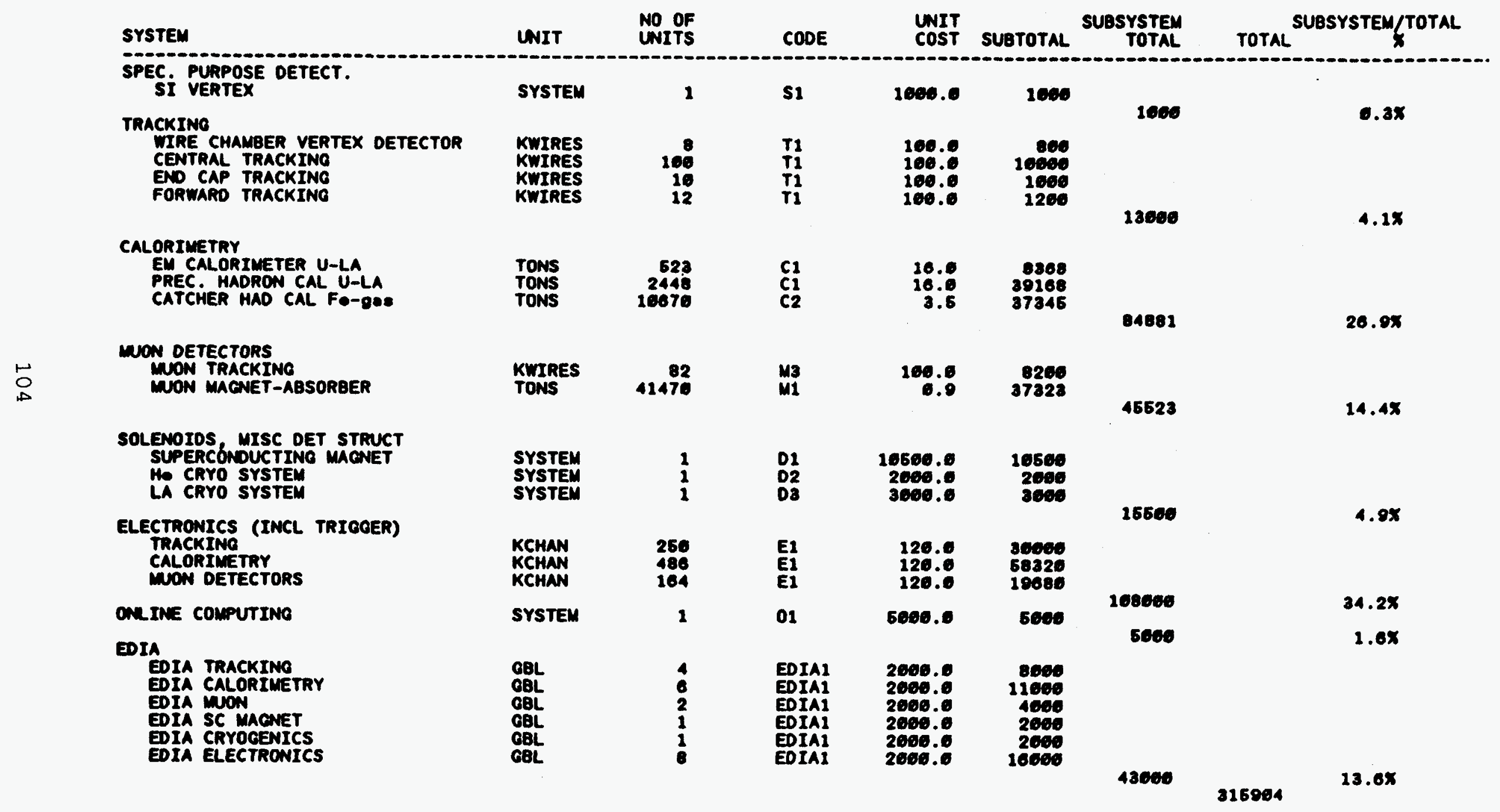


MODEl B (TABle 1)

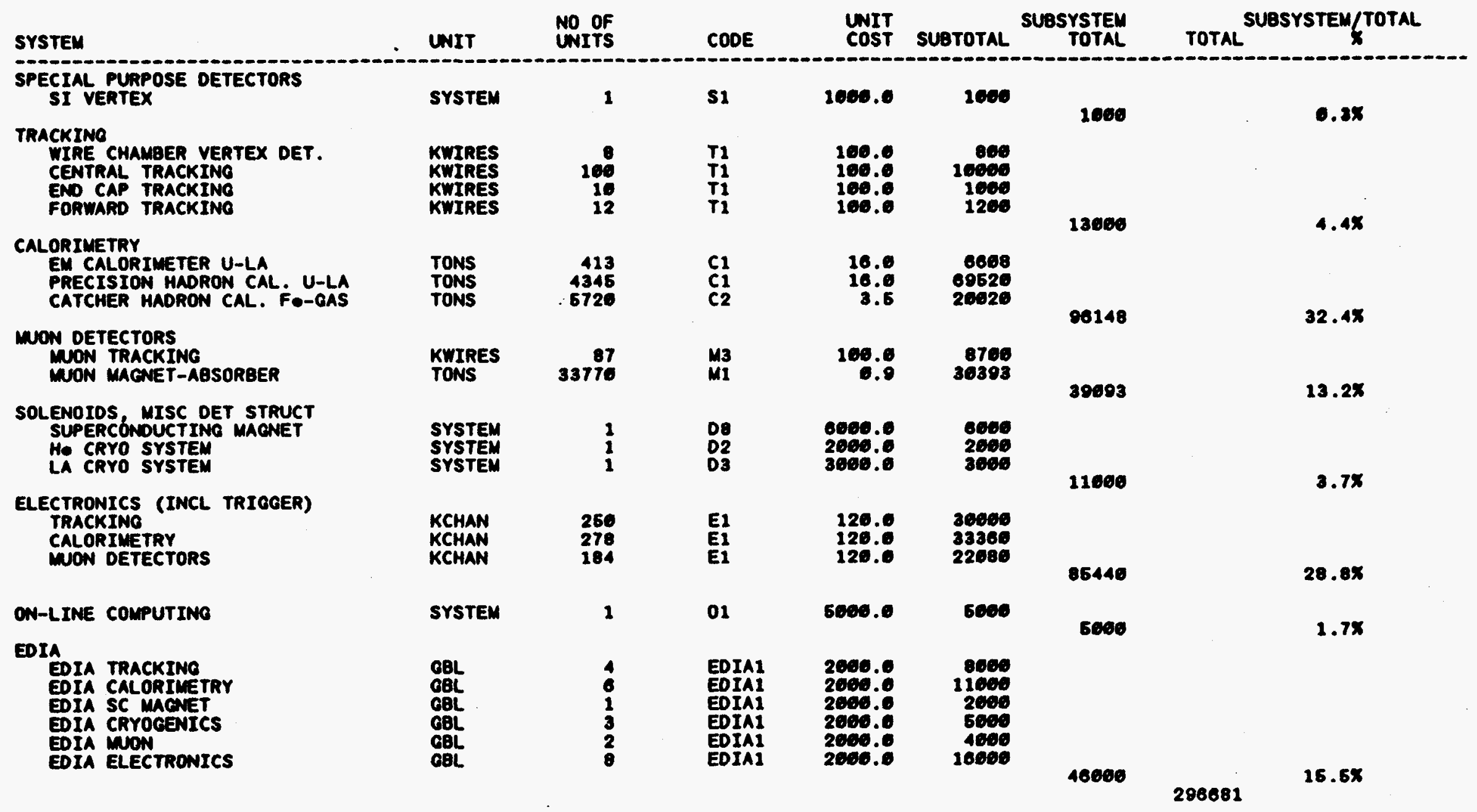


PRECISION MUON SPECTROMETER (TABLE a)

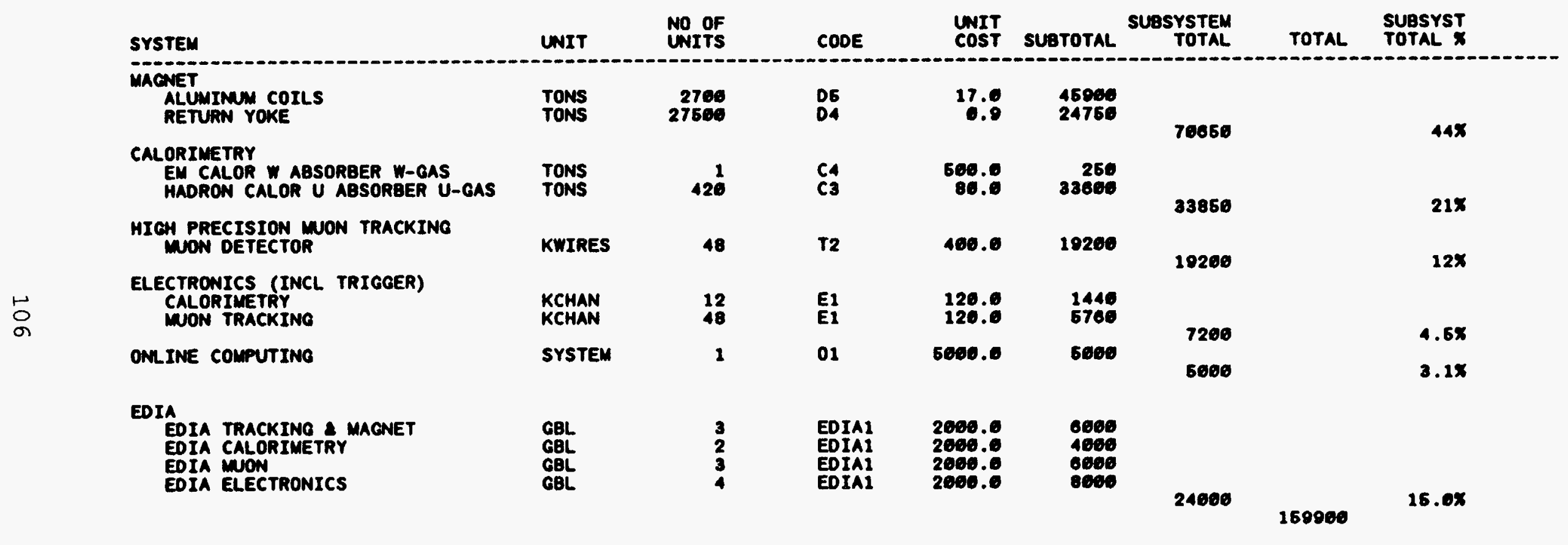


IRON MUON SPECTROMETER (TABLE 4)

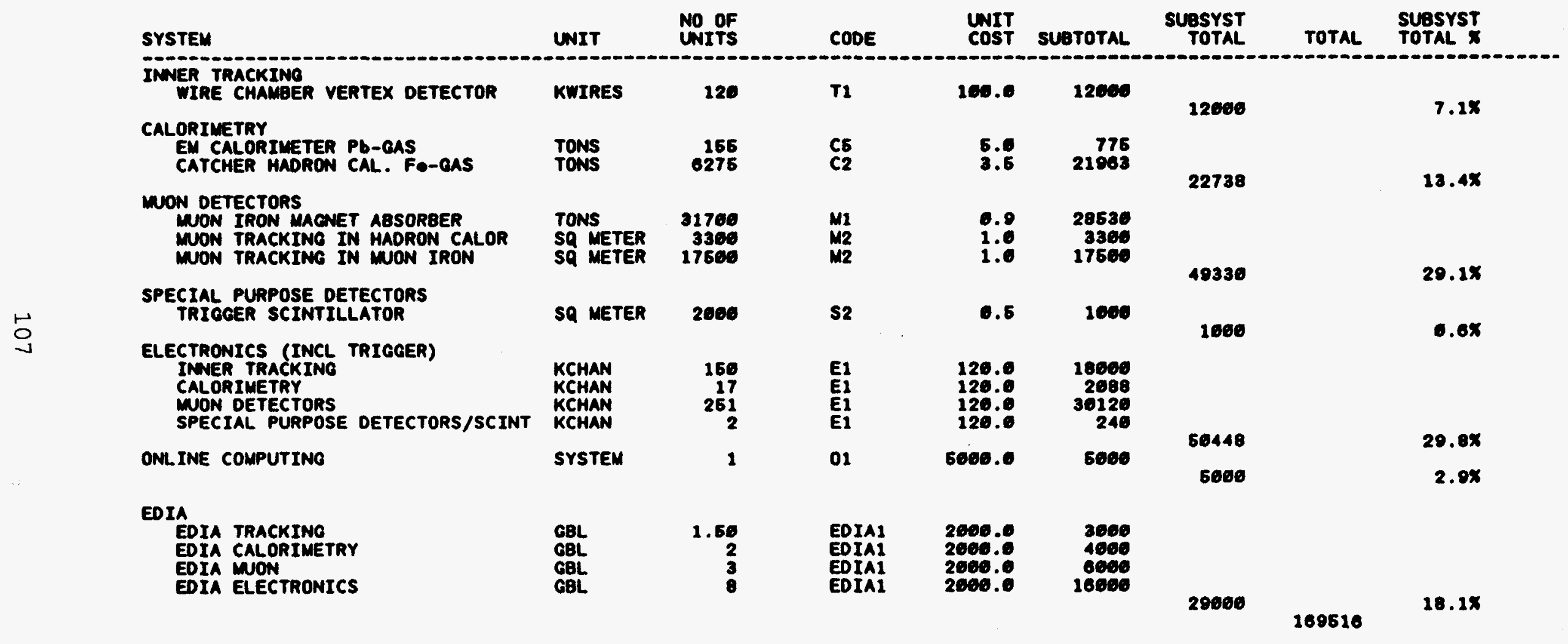


Forwaro detector (TABle 5)

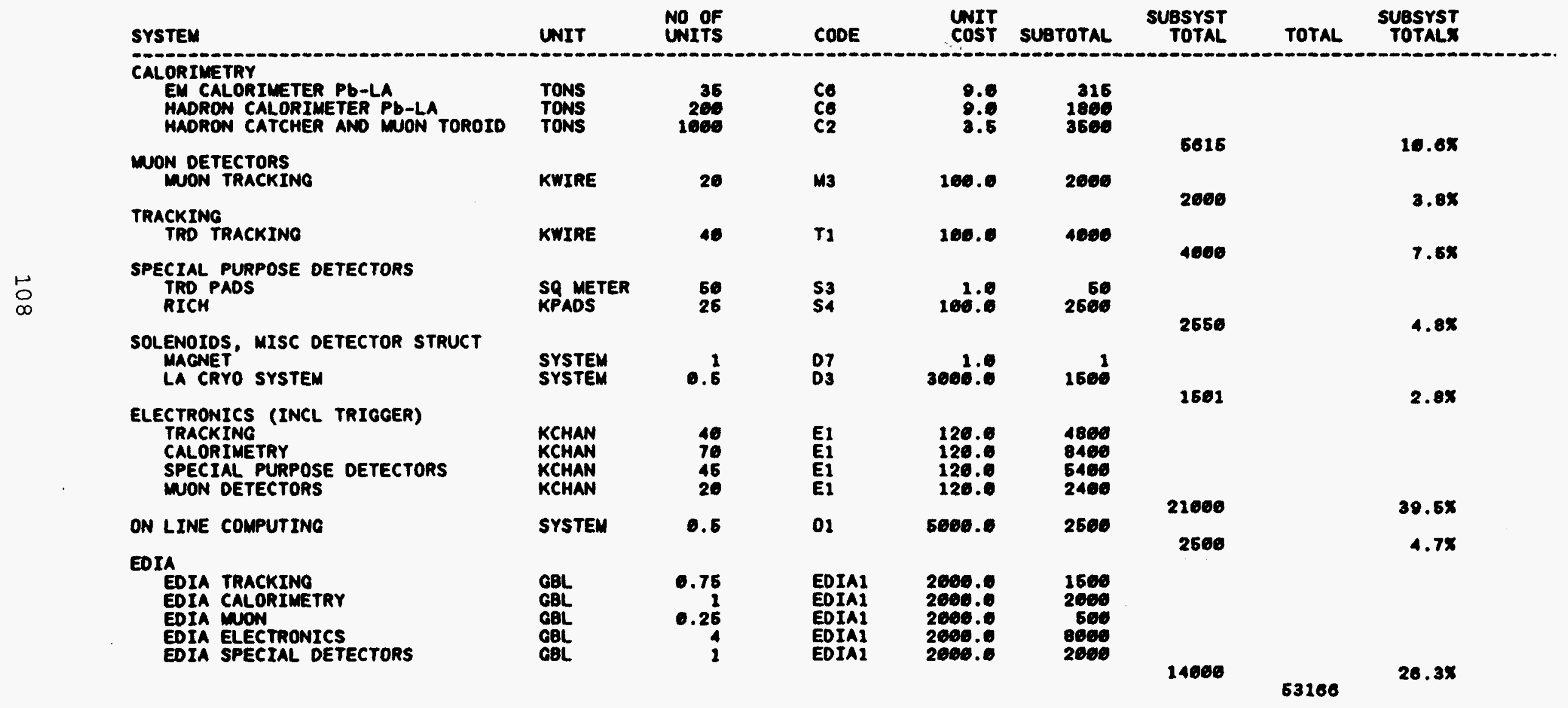




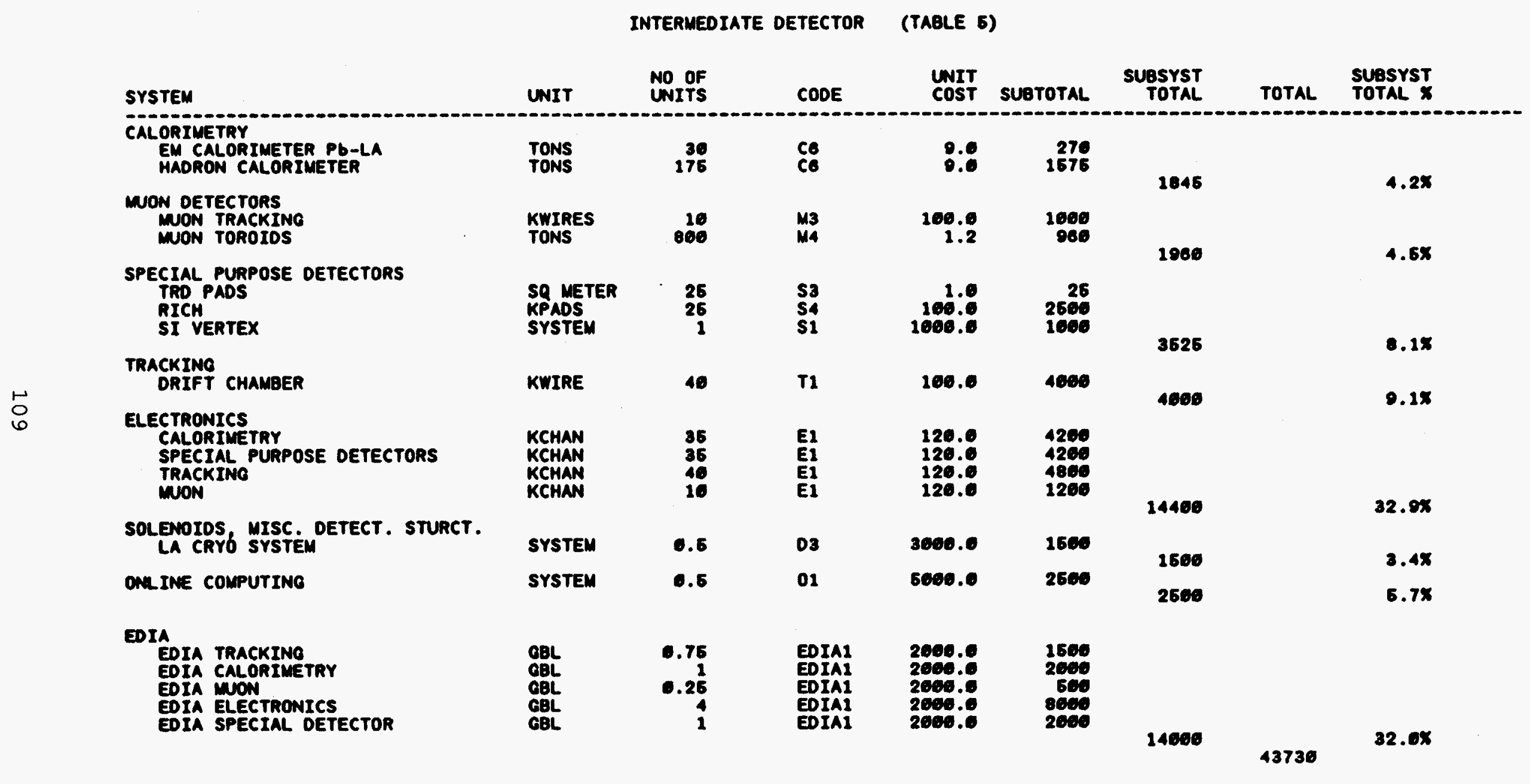


(TABle o)

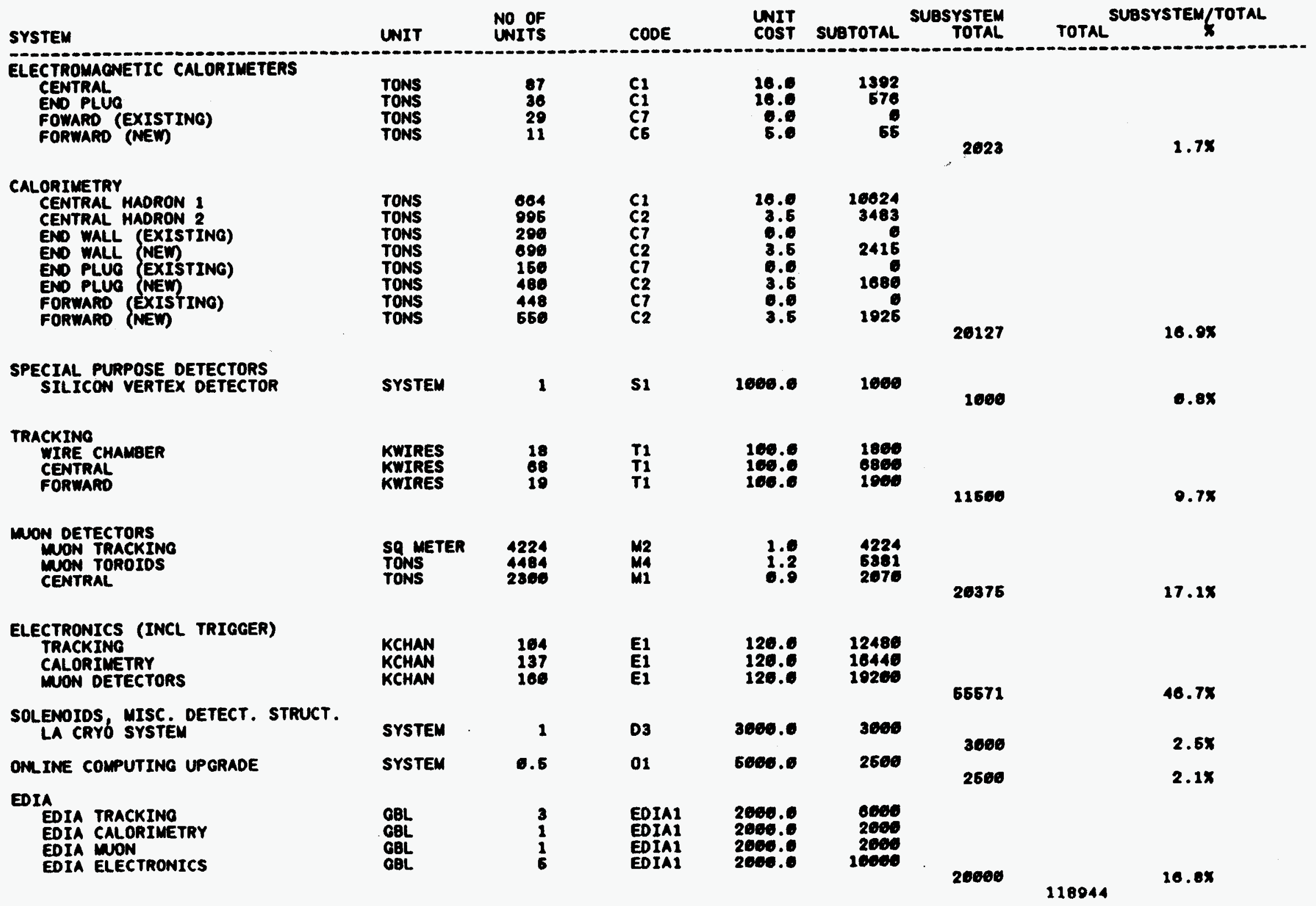


DO UPGRAOE (TABLE 8)

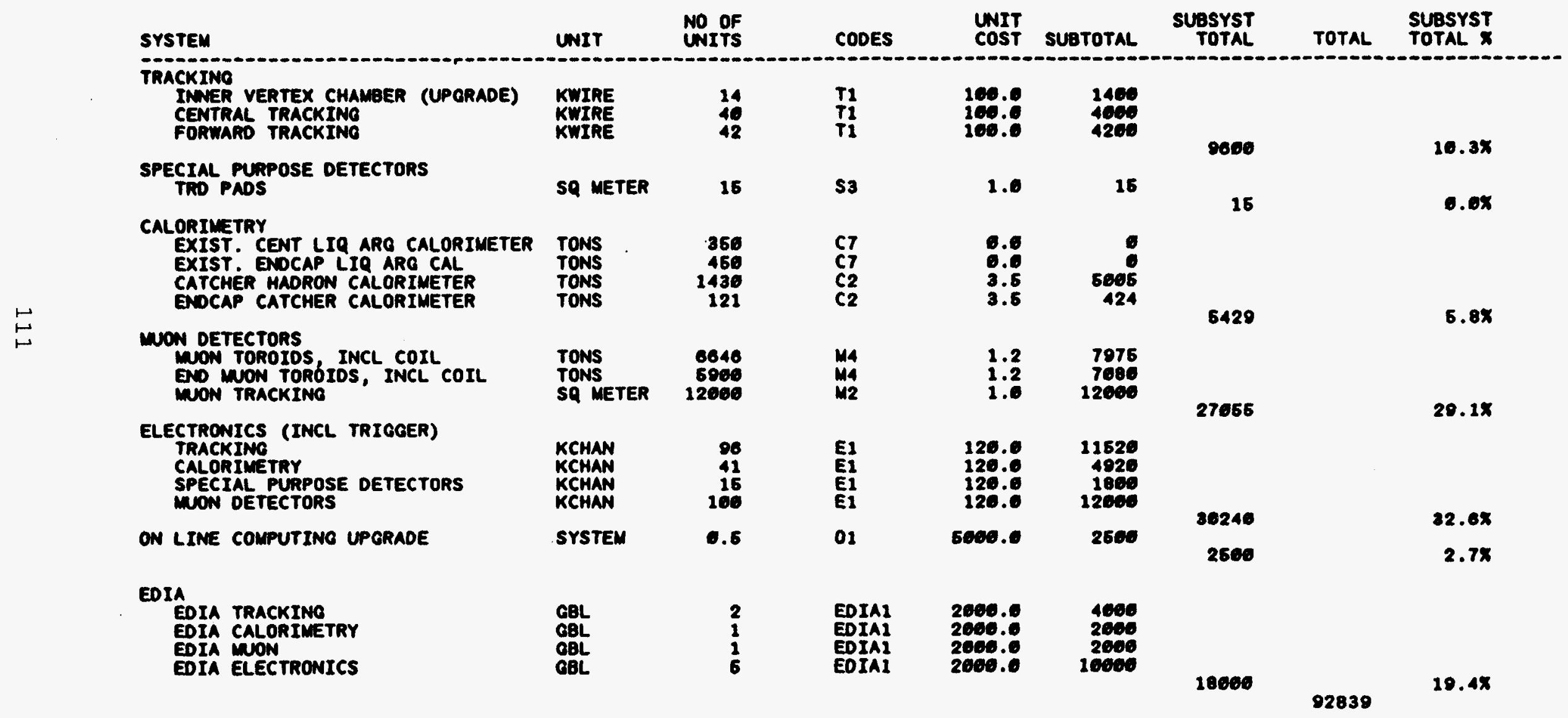




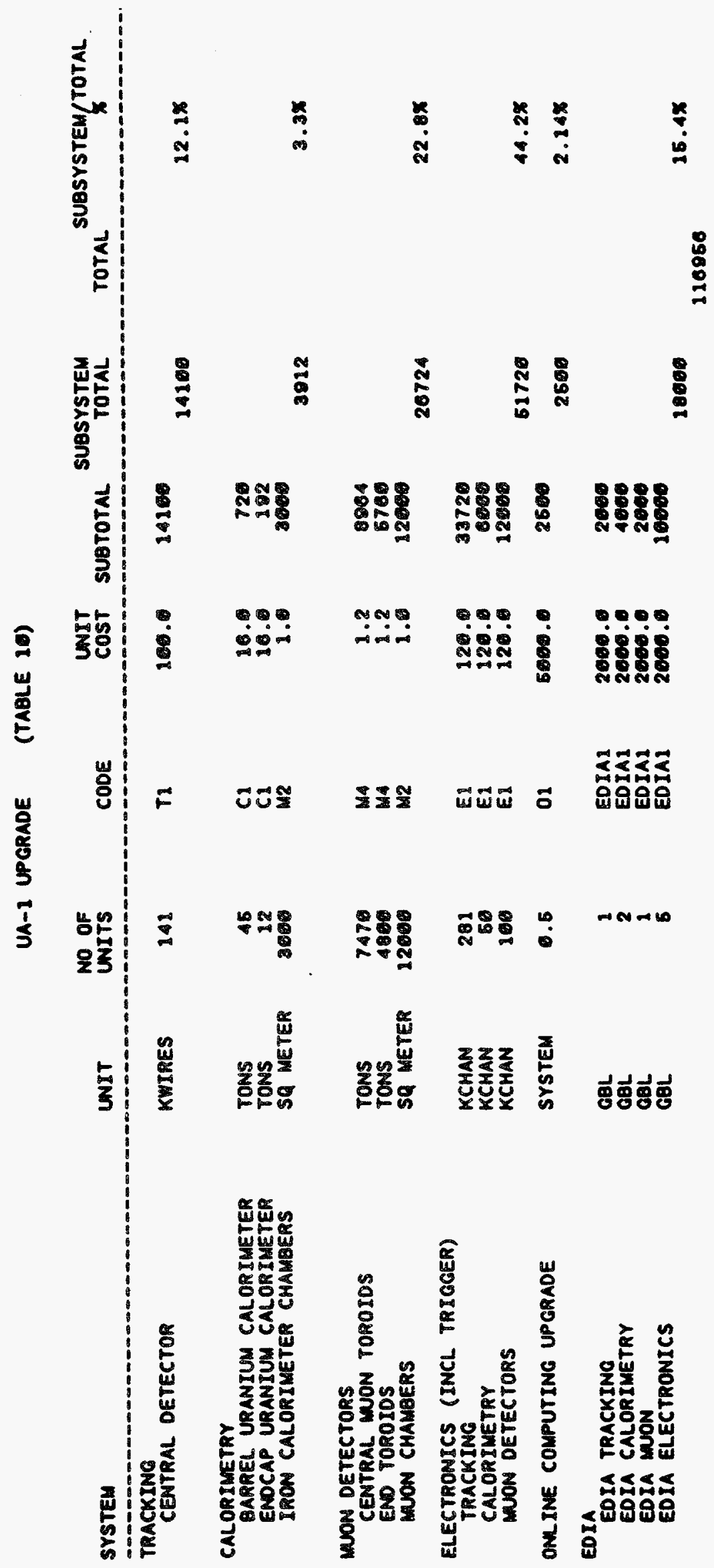


SYSTEM SUMMARY

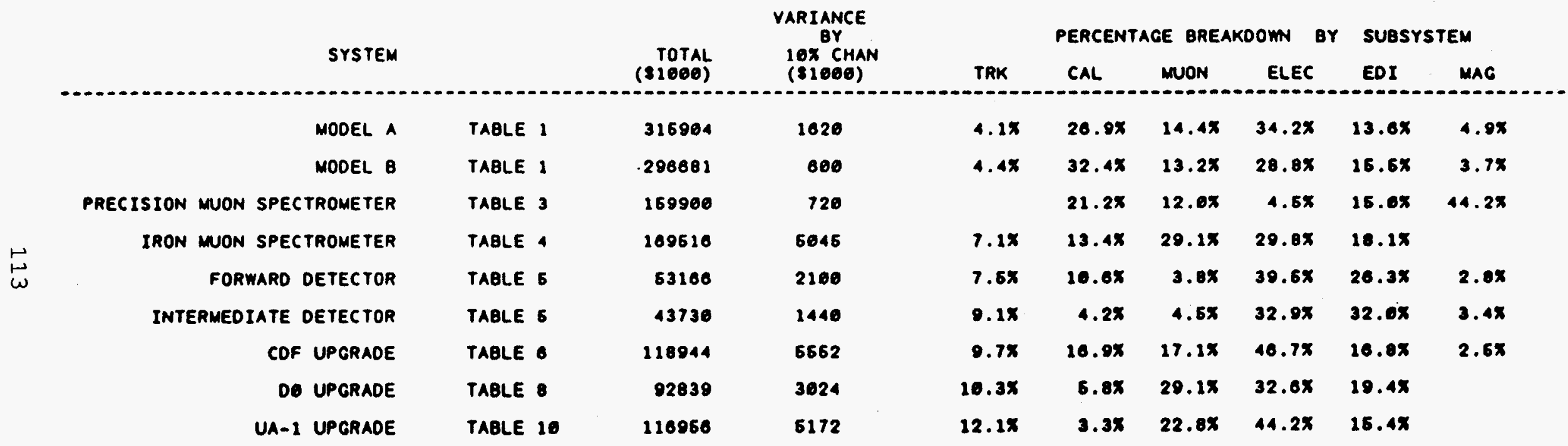




\begin{tabular}{|c|c|c|c|}
\hline SUBSYSTEM & UNIT & CODE & COST/UNIT \\
\hline 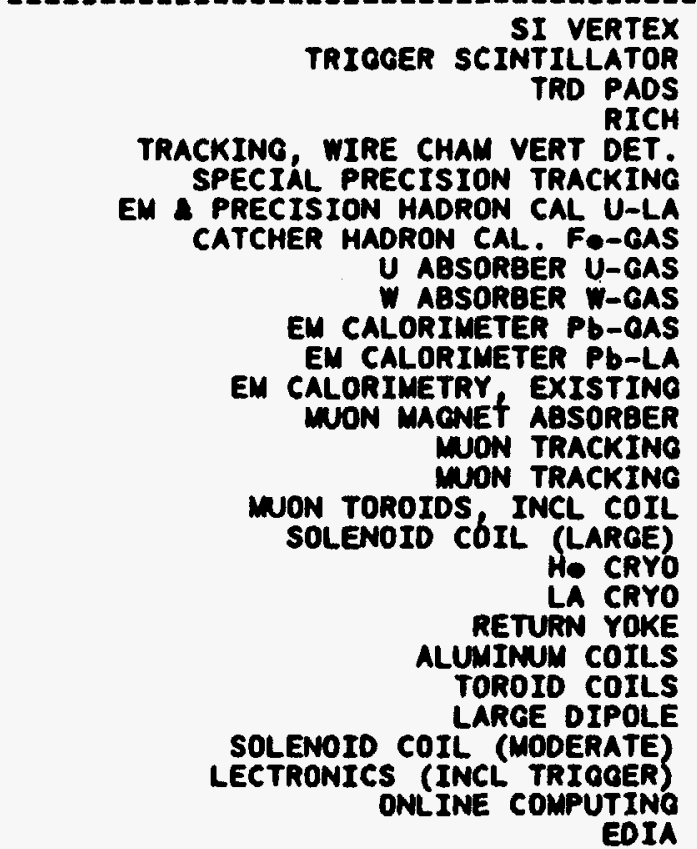 & $\begin{array}{l}\text { SYSTEM } \\
\text { SQ METER } \\
\text { SQ METER } \\
\text { KPADS } \\
\text { KWIRES } \\
\text { KWIRES } \\
\text { TONS } \\
\text { TONS } \\
\text { TONS } \\
\text { TONS } \\
\text { TONS } \\
\text { TONS } \\
\text { TONS } \\
\text { TONS } \\
\text { SQ METERS } \\
\text { KWWRES } \\
\text { TONS } \\
\text { SYSTEM } \\
\text { SYSTEM } \\
\text { SYSTEM } \\
\text { TON } \\
\text { TON } \\
\text { TON } \\
\text { SYSTEM } \\
\text { SYSTEM } \\
\text { KCHAN } \\
\text { SYSTEM } \\
\text { GBL }\end{array}$ & $\begin{array}{l}S 1 \\
S 2 \\
S 3 \\
S 4 \\
T 1 \\
T 2 \\
C 1 \\
C 2 \\
C 2 \\
C 3 \\
C 4 \\
C 5 \\
C 6 \\
C 7 \\
M 1 \\
M 2 \\
M 3 \\
M 3 \\
M 4 \\
D 2 \\
D 2 \\
D 3 \\
D 4 \\
D 5 \\
D 6 \\
D 6 \\
D 7 \\
D 8 \\
E 1 \\
01 \\
\text { EDIA1 }\end{array}$ & 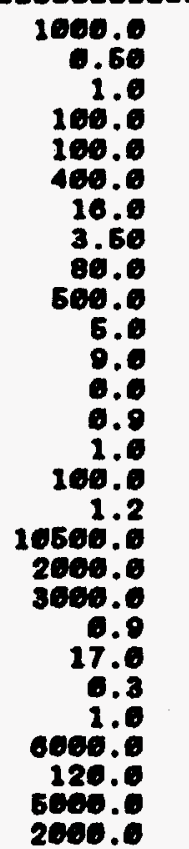 \\
\hline
\end{tabular}


REPORT OF THE OFF-UNE COMPUTING ADVISORY PANEL

May 1986 


\section{Introduction}

The off-line Computing Advisory Panel was charged by Maury Tigner to provide the SSC Central Design Group with an estimate of the probable cost of the initial computing facilities at the SSC. The membership of the panel is given in Appendix $A$. The specific charge to the panel was:

"The Off-line Computing Advisory Panel will estimate the cost of the initial computing facilities at the SSC. In estimating the computing power necessary to process the data from the SSC detectors, the Panel will take the recommendations of the Detector Cost Model Advisory Panel as providing the appropriate scenario for the initial SSC detectors. The Panel should not concern itself with on-line computing facilities associated with each detector and necessary for its data acquisition. The computing power recommended, however, will allow for appropriate simulation studies necessary for the design of the detector and subsequent analysis of the data.

"The Panel should assume that first pp collisions will take place in 1994. The Panel should use its best judgment in estimating the trends in the future developments in the computing area and in the associated costs. The costs should be expressed in FY86 \$."

In this report, we distinguish between two types of computing. All interactive computing, including program development, physics analysis, engineering calculations and library management, use a conventional computing system. The central processors are expected to be powerful mainframe computers. To accommodate engineering and database applications, these computers will likely have vector hardware and specialized database 
processors. The second type of computing is the reduction of raw data from the SSC detectors, and the Monte Carlo simulation needed to design the detectors and to calculate the detector acceptance. This computing will be done on a large array (farm) of small processors, each of which fully analyses a single event.

The computing requirement for the first type of computing is determined from the number of users of the system. The requirement for the second type is determined by the total number of events from the detectors and the analysis time to reconstruct tracks in the detectors. The assumptions which lead to the computing cost estimates are discussed in the report. The total cost for the initial computer facilities are as follows:

$\begin{array}{lr}\text { Mainframe Computer System } & \\ \text { Processor } & \$ 20.0 \mathrm{M} \\ \text { Storage Systems } & \$ 14.0 \mathrm{M} \\ \text { Software Products } & \$ 5.0 \mathrm{M} \\ & \\ \text { Total } & \$ 39.0 \mathrm{M} \\ & \\ & \\ \text { Communications and Printing } & \\ & \\ \text { Communications Front-end } & \$ 2.0 \mathrm{M} \\ \text { Terminals and connections } & \$ 2.0 \mathrm{M} \\ \text { Workstations and connections } & \$ 0.2 \mathrm{M} \\ \text { HEPNET interface } & \$ 0.9 \mathrm{M} \\ \text { Printing } & \$ 4.5 \mathrm{M} \\ \text { Digital PBX (voice/data) } & \$ 4.0 \mathrm{M} \\ \text { Installed cable/optical fiber } & \$ 17.6 \mathrm{M} \\ \text { Total } & \\ & \\ & \\ \text { Processor } & \\ \text { Farms } & \$ 14.0 \mathrm{M}\end{array}$


Annual Operating Costs (1994/95)

$\begin{array}{lr}\text { Manpower } & \$ 4.2 \mathrm{M} \\ \text { Hardware maintenance } & \$ 1.9 \mathrm{M} \\ \text { Software licenses } & \$ 1.2 \mathrm{M} \\ & \\ \text { Total } & \$ 7.3 \mathrm{M}\end{array}$

Software is expected to be a large part of the system cost. This includes the operating system and languages but, in addition, has many products that have traditionally been written for specific applications within high energy physics. The latter include library management and high-level graphics. Software will be an increasing fraction of the total cost of all computer facilities.

In order to provide computing for the design of detectors, and to provide computing for business and scientific applications, a smaller system will be needed before the installation of the system costed above. We estimate that the smaller system could be installed in FY1991 at a cost of \$8M. Annual costs would be $\$ 3.5 M$, including $\$ 0.6 M$ in new hardware acquisitions.

\section{Requirements}

A model for the initial complement of detectors is described in the DCMAP report. That model assumes four well-defined detectors. Other, smaller experiments are also expected but would likely have significantly less impact on the computer resources. The four detectors are the following:

1. A new magnetic $4 \pi$ detector designed to handle the full design luminosity for the study of physics at the highest mass scales made accessible by the SSC. This detector is expected to have $650 \mathrm{~K}$ to $1050 \mathrm{~K}$ electronics channels. The average event record is 200 to 1000 kbytes. 
2. A spectrometer for high energy muons designed for the highest luminosity operation. This may be a precision spectrometer or an iron spectrometer. The total number of electronics channels is approximately 400K.

3. An upgraded $4 \pi$ detector with forward spectrometer. This device is expected to operate at about $10 \%$ of the design luminosity. The forward spectrometer has $185 \mathrm{~K}$ channels; the upgraded central detector has $250 \mathrm{~K}$ to 400K channels.

4. Total cross-section and elastic scattering. This will be a small and specialized experiment with few detector elements.

To estimate the total computer power needed for the analys is of event data from the new $4 \pi$ detector, we begin with the analysis time for the UAl detector. This was reported by $D$. Linglin at the Workshop on Triggering, Data Acquisition and Computing. The analysis takes 4 seconds of $3081 \mathrm{~K}$ time per event (using both processors). This would correspond to 80 seconds on a VAX-11/780. For our estimates, the VAX is taken as 1 Mip.

We have assumed a trigger rate of 1 per second for the $4 \pi$ detector. Recent workshops have indicated that this rate can be achieved with the trigger systems that are planned for SSC detectors. To estimate the time to analyze a trigger, we have extrapolated for the UAT time to higher multiplicity. We assume that half the time is linear in multiplicity and half is quadratic. The multiplicity ratio was estimated by comparing Monte Carlo simulation of $35 \mathrm{GeV}$ jets at the SPS and $1 \mathrm{TeV}$ jets at the SSC. This should provide a worst case estimate and allow for the effects of overlapping events in the detector. The analysis time for SSC events is estimated to be 2 minutes on the $3087 \mathrm{~K}$ or 2500 VAX seconds. Assuming a rate of 1 event per 
second, the total computing power must be approximately 2500 Mips or VAX 780 equivalents. The effect of overlapping events is not expected to increase this number significantly.

The muon detector and the upgraded detector with forward spectrometer each have approximately half the number of channels of the new $4 \pi$ detector. We have assumed that each will require half the analysis time. The total need for all smaller experiments is taken to be the same as these two. We conclude that the nominal capacity required is 2.5 times the 2500 Mips (or VAX 780s). Assuming an SSC duty cycle of $70 \%$, we require 5000 Mips for the analysis of all experiments. To accommodate Monte Carlo simulation and other event processing, we have taken the total requirement as 10,000 Mips. This computing is to be provided by the farm of microprocessors or emulators described below.

The efficient development of production analysis code requires that the turn-around time on a single event be reasonably short. In the case of the largest detector we have estimated that this analysis will require $\sim 2.4 \times 10^{9}$ instructions or 24 seconds on a 100 Mips processor. After a set of changes is made in an analysis routine, the entire cycle of compiling the routine, relinking the program, reanalyzing and graphically displaying the target event, should take no more than about a minute. This requires a single processor in the 50-100 Mips range which would also allow batch processing of small samples of events with a turn-around time of about 1 hour, another desideratum of the system.

In addition to the computing for code development there are also detector design and engineering tasks which cannot readily be divided so as to run on an array of independent small processors. Examples of these are magnetic field calculations and finite element analyses. This type of calculation will benefit from the avallabllity of the most powerful single processor possible. 
The requirements on total installed capacity for high level computing are set by the expected peak (daytime) demand of some 500 users. In addition to the activities mentioned above, these users will be engaged in graphics-intensive interactive data analysis, development of non-production code, studies of detector performance, calibration calculations, document preparation, electronic mail, etc. At present h.e.p. installations during periods of peak demand $\sim 0.07$ Mips is available to each user. We estimate that this figure will need to increase to $\sim 0.4$ Mips/user at the SSC. This estimate reflects increases in demand to serve not only the tasks discussed above but completely new activities which are difficult to specify at present but which will no doubt become important. These may include, for example, Artificial Intelligence (AI) or new forms of statistical analysis. The total required capacity is then 600 Mips.

Estimates of the need for storage capacity are somewhat scenario dependent. We distinguish three types of data storage: immediate - random access with tens of millisecond retrieval; intermediate - sub-minute response, possibly random access; and archival - several minute response, probably sequential.

Archival storage at the rate of $2.5 \times 10^{13}$ Bytes/year will be needed for the raw data. This is equivalent to 167,0006250 bpi tapes, a somewhat daunting figure but one which could be accommodated if necessary. However, significant improvements in archival storage media can certainly be expected by 1995. If the 1995 version of archival media features automatic retrieval, intermediate storage may not be necessary. If not, one would like to keep about a one month buffer or 2 TB of raw data on intermediate storage along with an equal quantity of processed data and a year's worth of DST-level data (another 2TB). This total of 6TB would fit comfortably on a 10TB system, leaving space for a larger buffer and for other uses. 
On the other hand, if immediate storage becomes cheap enough to afford 10TB capacity, this would also obviate the need for an intermediate medium. In the absence of this use, extrapolation of current trends at HEP facilities indicates a need for 1-2 GB/Mip, or 0.6 to 1.2 TB of disk-type storage.

Internal networking needs depend on the computing distribution model selected and on the stategy for raw data collection. On the assumption of completely centralized computing and data recording, it would be necessary to have links capable of at least the data recording speed (IMByte/sec) between the IR's and the computer center. If no local buffering were available these links would need to run at 3 or $4 \mathrm{MByte} / \mathrm{sec}$ to avoid introducing dead time. This is probably the highest bandwidth requirement. There would also need to be links to local offices and IR's capable of serving graphics work-stations. These would require about $1 \mathrm{mbit} / \mathrm{sec}$. This would of course be sufficient to serve the needs of editing terminals as well. Connections to remote institutions would need to run at 56 kbaud at least. One Mbit/sec lines would be preferable if they could be afforded since they would allow transmission of graphics data and small data files.

\section{Conventional Computing}

In planning the conventional computing, we have assumed a single computing center. The alternative of several local facilities seemed to have no benefits and would increase the total operating costs of the facility. We assume a user base of approximately 2000, with 500 active at any one time. The users will include many who are computing at the SSC but are at their home institution. We have included the necessary networking hardware but we do not include the cost of network links or facilities at any of the universities or other laboratories. 
The total computing and storage requirements are defined above. We have assumed GSA list pricing and have used recent experience to estimate the decrease in cost per year. The guidelines anticipate a 20-30\% decrease per year for CPUS, $40 \%$ for storage and $12 \%$ for communications. The price of total systems drops by a factor of two in approximately 5 years.

The hardware configuration will be required to support the following environments:

1. Program development including testing, debugging and some production of Physics Analysis. The production would be limited to analys is not amenable to farm processing as for example in event analysis requiring special graphics. The number of users is anticipated to be 1500-2000 with up to 500 simultaneous locally or remote. Fast batch turnaround with less than 1 hour turnaround for 100 event samples is required.

2. Engineering computation including civil construction, mechanical, electrical and robotics. This use would generally be require commercially obtained software packages. The anticipated number of users is 100, mostly local, of whom 50 are professional engineers.

3. Document preparation. Applications in this area include word and text processing with integrated graphics. The products are publication quality documents or documentation. The area would also include library-like services such as references and access to libraries from on and off-site. We anticipate 40-50 users.

4. Administrative Data Processing (ADP). This includes payroll, inventory, legal, accounting, accounts reveivable, general ledger, stores, and procurement. This will use commercially purchased or public domain products. The expected user base is $40-50$. 
The hardware configuration required to support this computing environment in 1994/95 includes the following:

1. CPUs of at least 100 Mips each will be required. A vector component would be desirable for some commercial codes. Specialized database processors, now available as add-ons, should be available as standard features. The 600 Mip Central Processor is expected to cost $\$ 20 M$.

2. Data storage will utilize a two or three tier scheme. One possible model is based on these components:

Retrieval Time

a) Immediate

b) $<1$ minute

c) Minutes to Hours

\section{Media}

Disk ( 1000

Mass - (Hi Density Cartridge/Optical)

Archival

(Cartridge/Optica l)

$$
\text { Qty. }
$$

Cost

$\$ 7 M$

$\$ 5 M$

25TB

$\$ 2 M$

TOTAL
$\$ 14 M$

The total capacity required is rather clear but the division between the types of storage, and corresponding retrieval time, is less certain. This is an area where the technology and market will determine the optimum solution. Recent attempts to provide intermediate and tertiary storage with low-cost media have not been particularly successful. If the technical alternatives are available, a two tier system is preferable.

3. A communications front end is required for connecting terminals and workstations as well as local and remote printers. This cost is estimated at $\$ 2 M$. 
4. Printers of 100 page per minute capacity are required for central and remote users. We assume one such device for 150 technical or professional users. Devices capable of 30 pages per minute will be installed in areas with lower user density and at each intersection region. The cost of printing is summarized as:

High Speed

$\begin{array}{ll}\text { Computer Center } & \$ 100 \mathrm{~K} \\ \text { Document Preparation } & \$ 150 \mathrm{~K} \\ \text { Medium Speed/High Resolution Color/Graphics: } & \\ 2 \text { O } \$ 40 \mathrm{~K} \text { for each of 4 Interaction Regions } & \$ 320 \mathrm{~K} \\ 10 \text { in the campus area C \$35k } & \$ 350 \mathrm{~K} \\ & \\ \text { TOTAL } & \$ 920 \mathrm{~K}\end{array}$

5. We include the costs of terminals and workstations and the cost of connecting these to the communications front end computer. The local area network is assumed to use a fiber optic network capable of 1 MegaByte per second. The cost of the fiber optic cables is included as a separate item.

a) 2000 "standard" terminals

C $\$ 1500+\$ 500$ for connection $\$ 4 M$

b) Work Stations

50 Physics Workstations (a \$20K 5 o each IR 30 a CAMPUS

25 Engineering Workstations @ \$40K mostly CAMPUS based

c) 'HEPNET' Interface

56 KB Access for 125 remote users \$200k

d) Phone System 3500 Instruments

Switch Hardware/Sof tware

e) Cable Plant Back Bone 
Software for the computer system includes the operating system and languages as well as additional packages for library management, database management, graphics and Computer Aided Design. Most products have an annual maintenance cost as well as the initial license price. The software packages are itemized as follows:

Product

Operating System

Utilities

Archive

Data Base Products - Physics

Electronics

Language/Debuggers

Applications Software Generators

Source Code Manager

AI Tools

Graphics Packages - Application Presentation

CAD

ECAD

Robotics

CAM

Project Management

Text Processing

Network Products - Gateway,

File Transfer, Connect

Math \& Stat

On Line Manuals

Telecom Tools

On-line Consult Tools

Library Service
Capital

Annual Operating

$\$ 500 k$

$\$ 400 k$

$40 k$

$\$ 400 k$

40k

$\$ 500 k$

50k

$\$ 200 \mathrm{~K}$

20k

$\$ 240 K$

$24 k$

$\$ 400 k$

40k

$\$ 200 k$

20K

$\$ 200 \mathrm{~K}$

20k

$\$ 200 k$

20k

$\$ 200 \mathrm{~K}$

20k

$\$ 200 k$

20k

$\$ 200 \mathrm{~K}$

$20 k$

$\$ 300 \mathrm{k}$

30k

$\$ 200 \mathrm{~K}$

$20 k$

$\$ 800 k$

$80 k$

$\$ 160 \mathrm{~K}$

$16 k$

100K

$\$ 100 k$

10k

$\$ 100 \mathrm{~K}$

10K

$100 \mathrm{~K}$ 
The annual operating costs of the computing facility have been estimated based on experience in the SLAC and Fermilab Computer Centers. The details of the staffing requirements are described in Appendix $B$. The total requirement is 58 Professionals and 42 Technical or administrative. The estimated cost is $\$ 4.2 M$ per year. The hardware maintenance is estimated to be $3 \%$ of the capital cost, or $\$ 1.9 M$. The cost of software leases and maintenance is itemized above. The total operating cost is $\$ 7.3 \mathrm{M}$.

The initial costs include floor space for computers, mechanical systems and offices, as well as the manpower for the initial computer system purchase. Those needs can be summarized as follows:

Facilities

Computing Center Raised Floor Mechanical Support Space office space

Computer Acquisition

Requirements definition leading to RFP 6-8 months with 6-8 FTE's

RFP Production

3-4 months with 6-8 FTE's
$48 \mathrm{~K} \mathrm{Sq} \mathrm{Ft}$

$8 \mathrm{~K} \mathrm{Sq} \mathrm{Ft}$

$12 K \mathrm{Kq} \mathrm{Ft}$

The panel was asked to size and cost a computing capability for general use prior to $1994 / 95$. This system would be specified soon after approval of the SSC project and the system be selected to make the software conversion as easy as possible. We assume that this system would not be part of the 1995 configuration except possibly as the administrative computer.

We estimate that the initial configuration would have a capacity of 40 Mips and would cost $\$ 8 M$. To satisfy increasing demands, there would be new hardware purchases of approximately $\$ 0.6 \mathrm{M}$ per year for the three fiscal years. The software costs would be $\$ 300 k$. 
The annual operating costs for the three year period are expected to be $\$ 2.9 M$. This includes professionals $(\$ 1.9 M)$ and technical support $(\$ 0.7 M)$ as well as hardware maintenance $(\$ 0.3 \mathrm{M})$ and software licenses $(\$ 0.03 \mathrm{M})$. The manpower estimates are the following:

$\begin{array}{lr}\text { Administrative } & 4 \\ \text { Operations } & 12 \\ \text { Network (Termina 1s) } & 11 \\ \text { User Support } & 6 \\ \text { Special Products/Technology } & 8 \\ \text { Systems } & 9 \\ \text { ADP } & 5 \\ \text { Phones } & 6\end{array}$

Annual Operating Costs (1994/95)

$\begin{array}{lr}\text { Manpower } & \$ 4.2 M \\ \text { Hardware maintenance } & \$ 7.9 M \\ \text { Software licenses } & \$ 1.2 M \\ & \\ \text { Total } & \$ 7.3 M\end{array}$

Software is expected to be a large part of the system cost. This includes the operating system and languages but, in addition, has many products that have traditionally been written for specific applications within high energy physics. The latter include library management and high-level graphics. Software will be an increasing fraction of the total cost of all computer facilities.

In order to provide computing for the design of detectors, and to provide computing for business and scientific applications, a smaller system will be needed before the installation of the system costed above. We estimate that the smaller system could be installed in FYl991 at a cost of $\$ 8 M$. Annual costs would be $\$ 3.5 M$, including $\$ 0.6 M$ in new hardware acquisitions. 


\section{Processor Farms}

As described above, a very large fraction of the computing load at the SSC does not require general purpose mainframe computing. This large part of the load includes event reconstruction and Monte Carlo event generation, which share the characteristics that the same computer program is run repetitively on many millions of events, that the computing on each event is essentially independent of the computing on other events, and that the amount of CPU time needed for each event is much greater than the amount of input-output (that is, the job is compute bound).

These characteristics allow this computing to be performed on processor farms, defined as a collection of large numbers of identical processors running identical programs on different events. Because of the inherent parallelism of the computing problem at hand (due to the event oriented nature of the analysis), extensive use can be made of relatively trivial arrays of processors working in parallel, without the complications that more general purpose parallel processing involves. This allows the choice of extremely cost effective methods of computing for this part of the computing load.

Within this general framework of processor farms, there are a number of different approaches that can be taken. These include the use of emulating processors, the use of commercial microprocessors (on either specially designed or commercial CPU boards), and the use of commercial special purpose parallel processing systems. Examples of all three of these approaches either exist or are under construction at the present time. The different approaches share many features in common, particularly the need for support software, links to a host system, and program development environments. Consideration of various approaches allows us to make conservative extrapolations for the cost and performance of systems that will be available on the SSC time scale. 
The first approach, of emulating processors, allows the design of higher performance processors than commercial micros, allows bit-for-bit comparisons with a mainframe to verify the correct operation of the emulating processor, and also benefits from the advanced stage of mainframe compiler technology by emulating mainframe instruction sets. These advantages are offset by the additional engineering design necessary to build emulating processors and to upgrade systems from one generation to the next. If future needs require processing nodes of higher performance than obtained from cheap commercial CPU's, then this approach or variations will be essential.

The emulator approach was the first one to be tried. An example of this approach are the $168 / E$ and $3081 /$ E projects developed initially at SLAC and now by a SLAC/CERN collaboration. These machines emulate the IBM 370 instruction set. 168/E processors have been in use around the world since 1980, with the first systems giving a performance of about 16 MIPs with 8 or so processors. The first generation $168 /$ Es are currently being replaced in both on-line and off-line applications by the newer generation $3081 / E$ processors. These systems achieve performance of 4-5 MIPs, and a system with 2 MBytes of memory costs roughiy $\$ 15,000$.

Future generations of emulators would involve redesigns using faster integrated circuits and/or custom VLSI, and would most likely result in more powerful machines with more memory at roughly the same cost as current systems. The current generation of emulators are modular, allowing portions of the machine to be upgraded without a total redesign. Future systems could again emulate IBM mainframes or other suitable instruction sets. Based on the experience in moving from $168 / E$ to $3081 / E$ processors and on the expected improvements in chip performance, we can anticipate performance improvements of roughly a factor of 4 in 5-8 years, giving a cost of order $\$ 1000 / M I P$. 
The second approach, of using commercial 32-bit microprocessors, can be extremely cost effective because of the "commodity" nature of the CPUs used and their consequent low price. This approach can involve buying chips and building your own CPU boards, or buying commercial CPU boards. The recent development of 32-bit micros by several vendors (including National Semiconductor, Motorola, AT\&T, and Inte1) together with the accompanying development of real Fortran-77 compilers for these micros, has made this approach viable. Such 32-bit micros represent the first generation of commercial CPU chips that can realistically run large physics analysis codes. These advantages are offset by requiring more processors and memory for a given level of total capacity.

One example of this approach is the multi-microprocessor system currently being assembled by the Advanced Computer Program at Fermilab. This project makes use of Motorola 68020 and AT\&T 32100 processors. It has produced CPU boards of roughly 1 MIP in performance, including floating point co-processors and 2 MegaBytes of onboard memory, on standard commercial VME bus cards at a cost of about $\$ 2500$. These boards, designed at Fermilab, are now being made available commercially. The initial project will assemble 128 processing nodes in a system to be used for off-line event reconstruction, while a second system will be used by CDF for an on-line third level trigger processor.

The future of this approach will most likely involve CPU boards of roughly comparable price with significantly higher performance. Processor clock speeds, processor architectures, and improved Fortran compiler optimization can be expected to yield an overall factor of 8 performance improvement over the next 5-8 years, while memory technology will allow at least 8 MBytes per board at the same price as existing 2 mBytes. Thus we can anticipate an 8 MIP CPU with 8-16 MBytes of memory for $\$ 2000-2500$, or roughly $\$ 300 / M I P$. 
Another example of this approach, using commercially designed CPU boards, is the multiple Micro-VAX processor system being constructed by the DO collaboration for use as a high-level trigger processor. This project makes use of commercial Micro-VAX II CPU boards, interconnected by Ethernet. Their initial system will have 50 such processors. Current boards achieve a performance of 1 MIP at a cost of about $\$ 7500$.

The third approach involves full commercial multi-processor systems. This approach would obviously require the minimum amount of in-house hardware and software support, since packaged total systems would be purchased. However, such systems might be significantly less cost-effective than home-made systems depending on the aggressiveness of the commercial vendor's pricing. Such systems are already available commercially from such vendors as Encore, Elxsi, and Flexible Computer. If such systems prove to be a viable market, we would expect the entrance of other larger vendors (such as DEC and IBM) into this market segment.

At the present time, such commercial approaches are directed at more general parallel processing problems than the simple one processor one event approach that SSC computing can use. Thus, such systems usually involve fewer processors, more complicated interconnection schemes, and more dollars per MIP than the first two approaches. Only if such systems allow themselves to used be efficiently in the one processor one event mode will they be competitive to the home-grown approachs at the SSC.

The future of this approach is somewhat more difficult to estimate, since the prices of such systems are determined more by market forces than by costs. However, it is clear that many vendors will have CPU boards of 5-10 MIPs in performance in the next 5-8 years, and commercial vendors can achieve system costs in the same ballparks as home-made systems using either of the 
first two approaches. We can thus anticipate such systems being on the market with prices in the $\$ 1000-2000 / M I P$ range.

Besides the cost of the processing nodes themselves, there are a number of other costs associated with the farms that need to be considered. These include links to host systems, development environments, and hardware and software support. The costs of these components will vary depending on the approach taken, but we can give some general guidelines.

A link to a host system is necessary to provide the processor farm with I/0 facilities for data input and output, disk file access, graphics and printer capabilities, and job submission and monitoring. The host system can either be dedicated small computers associated with each farm, or can make use of a small fraction of the $1 / 0$ and CPU power already present in the central mainframe facility, whichever is more appropriate. In either case, the farms require mass storage devices to support to production jobs running on them in addition to those used by other jobs in the central facility.

A development environment is necessary for users to test and debug code on a multi-processor system before going into production on the full-scale farm. This environment should have miniumal impact on the throughput of production jobs on the farms. This might, for example, be additional farms with only a few processors on each or sufficient software support to run test jobs on a few processors and production jobs on the remaining processors within a single farm.

Hardware and software support will vary enormously depending on how much of the system is built or bought. However, this variation in cost is more than covered by the difference in price of the processing nodes; i.e., homemade $\$ 300 /$ MIP nodes will require more support than $\$ 1000 / M I P$ commercial processors. We will use a conservative figure of $\$ 1000 / \mathrm{MIP}$ for the cost of 
the nodes, which is expected to be adequate either to allow the purchase of commercial systems, or to pay for the additional in-house support needed for a home-made system.

There will be certain software development and support costs independent of the approach taken, however. Even if commercial multi-processor systems are used, there may be a need to provide system software that allows users to port application programs from uni-processor systems to the farm in an efficient manner. Software will also be needed to manage the farm systems, maintain job queues, monitor performance, etc. A software group of about five people will probably be required to develop farm support software and later to consult with and support farm users.

As with all computers, it would be beneficial to delay the purchase of the processor farms as long as possible. However, users will need to be developing code that will eventually run on the processor farms and running Monte Carlo programs 2-3 years prior to actual data taking. Thus, a development environment and a sufficient farm for Monte Carlo must be available on this time-scale, and while the entire farm complex need not be purchased until closer to the start of SSC data-taking, the farm architecture must be settled enough in advance. This will likely require detailed discussions with vendors as to their future plans to prevent the development of home-made systems that would eventually be surpassed by commercial systems. In any case, a farm support group should be in place 3-5 years before data-taking. It should be noted that overall cost savings can arise if the off-line farms are developed in conjunction with the on-line farms that may be used for high-level triggers in the detectors, even though it is not anticipated that any use would be made of the on-line farms for off-line production. 
In summary, to provide a total of 10 farms of roughly 1000 MIPs each (for example, each farm could consist of 1288 MIP processors), we require:

1. Processors: 10000 MIPs o $\$ 1000 /$ MIP

2. Host systems:additional mass storage capability and $1 / 0$ bandwidth to support the processing power of the farms.

$\$ 2 M$

3. Engineering and design: included in the purchase price of commercial systems. If systems are designed by SSC groups, the ED\&I is can be included in the $\$ 1000 /$ Mip.

4. System Integration and testing: $\$ 2 M$

5. Software support: A group of 5 people. This is included in the personnel estimates above, as are the operating costs for the farms.

\section{Conclusion}

We have estimated the cost of the initial computing facilities for SSC operation. The costs are $\$ 65.6 \mathrm{M}$ for hardware and $\$ 5.0 \mathrm{M}$ for sof tware.

The total operating budget for the computing facilities is estimated to be $\$ 7.4 M$.

These facilities will provide all necessary computing for the initial complement of SSC detectors.

We have also estimated the cost of a facility for use prior to $1994 / 95$. The initial system is expected to cost \$8M. The annual operating budget for this system is approximately $\$ 2.9 M$. 
Appendix A

off-line Computing Advisory Panel

Stewart C. Loken, Chairman

Lawrence Berkeley Laboratory

One Cyclotron Road

Berkeley, CA, 94720

Charles Dickens

Stanford Linear Accelerator Center

P.D. Box 4349

Stanford, CA, 94305

Jack Pfister

Fermi National Accelerator Laboratory

P. 0. Box 500

Batavia, IL, 60510

Irwin Gaines

Fermi National Accelerator Laboratory

P.0. Box 500

Batavia, IL, 60510

Paul Kunz

Stanford Linear Accelerator Center

P.O. Box 4349

Stanford, CA, 94305

Laurence S. Littenberg

Brookhaven National Laboratory

Upton, Long Island, NY 11973 


\title{
Appendix B
}

\author{
Requirements for SSC Central Computing Staffing \\ (Conceptua 1) \\ November 1985 - Revised $4 / 86$ \\ Jack Pfister \\ FERMILAB \\ Batavia, Illinois
}

\section{Summary/Abstract}

Given a computation center with $\sim 10,000$ MIPS supporting $\sim 1,000$ users, what are the staffing requirements?

Introduction

The attempt in this note is to list the functions and staff size required in a central computing or centrally supported computing complex. The organization assumes that although considerable computing power would exist (mostly for online) in the four interaction regions (IR) that there are functions and capabilities better performed outside the IR and in this model at a "central computing facility". What follows is one staffing approach, not necessarily optimal, with certain assumptions about numbers of computer systems, media, networks and system controls, that is, we would get the best technology may bring and what it takes to operate it.

From an end user support standpoint it is less clear, given the geography of an SSC, where and what the consulting support should look like and its Tocation. 
The Organization

The computing organization consists of seven groups. They are Administration, Computer Operations, Network/Telecommunications User Support, Operating System/Utility Support, Special Projects and Technology Assessment. The next sections provide some specifics for each group in terms of function and size.

Administrative Groups

Functions: Overall direction, strategic planning, liaison with DOE, budget control, clerical and secretarial support for the computing facility.

$$
\text { 8-10 people }
$$

Comment: May want to cover the technology assessment, computer security and related DOE management issues.

Computer Operations Group

Functions: Operate central complex consisting of probably 10 discrete computer systems, mass storage and media management, capacity planning, data management, media management, accounting, supplies, data entry and media conversion. Operations runs 24 hours/day, year round. Four shifts of operators are imagined.

$$
\text { 55-70 peop le }
$$

(Shift personnel may be $45-55$ of this number)

Comment: The level of systems integration, operations management tools (as part of the operating system), data media variety and topology will have considerable influence on the actual numbers. 
Network/Telecommunications Group

Functions: Provide local area communication on campus as well as wide area and national/international gateway communications. Do all capacity/configuration planning and management. Provide terminal repair service.

\section{8-20 people}

( $\sim 8$ of these are shift workers - 'console operators')

Comment: Analytically intensive for planning and administration, immature as an industry and extremely volatile. Can involve extensive negotiation with tariffed carriers and component vendors. Few turn key solutions are marketed. Long lead times for implementation.

User Support Group

Functions: Support end users. Provide access to computing resources through the support of users in development production of the HEP codes. Maintain computing library (documentation and other media). Provide training and consulting on HEP specific or general computing techniques. Provide training and consulting on HEP specific or general computing techniques. Provide software package specialists (engineering, graphics). Maintain "Work Station" support center for training users in the existing equipment as well as evaluating the new.

\section{8-33 people}

Comment: The physical location of the people doing the support is an issue. What and how much consulting and support can be done electronically? Should there be a strong bonding of user support people to the IRs? What about the sociology and "careers" of the support personnel? 
Operating Systems/Utilities Group

Function: Install and maintain vendor supplied operating systems and utilities packages. Modify/tailor/tune systems for performance and capacity optimization.

\section{2-18 people}

Comment: Again as for operations, the diversity and number of operating systems (vendors) which will be supported will drive this, augmented by the amount of local modification (specials) that are required or permitted. The management of very large data files and lots of them will be a major technical issue.

\section{Special Products Group}

Function: Research the requirements of the SSC community with the view to obtaining either commercially developed or in-house developed software products specific to HEP analysis and related activities.

$$
\text { 6-8 people }
$$

(core group which would be augmented during the products' development)

Comments: This is a consumer products group with a charter to develop generally useful HEP software products where not available from other sources. It is intended to serve broad HEP/SSC community interests, not individual, unique or momentary requirements. Developed, tested and documented products are turned over to the User Support Group for long term distribution, support and maintenance. NB: The number of staff for this effort may be too few based upon comments from others reviewing the draft. I would agree, but the projects for initial staffing plan should be at least tentatively identified. 
Technology Group

Function: Technology assessment of computing and telecommunication related hardware and software issues as they affect current computing, but with the emphasis on the future of SSC computing.

4-6 people

Comment: This group should provide advice to current acquisitions and operations, however, their main focus should be acquisition requirements near term ( $1-3$ years), medium term (3-5 years), and long term ( $5+$ years). The note in the Special Projects may apply here.

Bottom Line: Total staff between 130 and 165.

What is Not Addressed?

1. Administrative computing which must pay people, procure goods and service, track projects, generate reports to funding authorities...

2. Strategic issues such as the independence or interdependence of the IRs. We assume a legitimate need for the central computing roles described above and have not looked at alternatives.

3. Data Acquisition Computers - their management and support.

4. Support for user terminals, work station, PC, in the form of installation, maintenance and repair. 